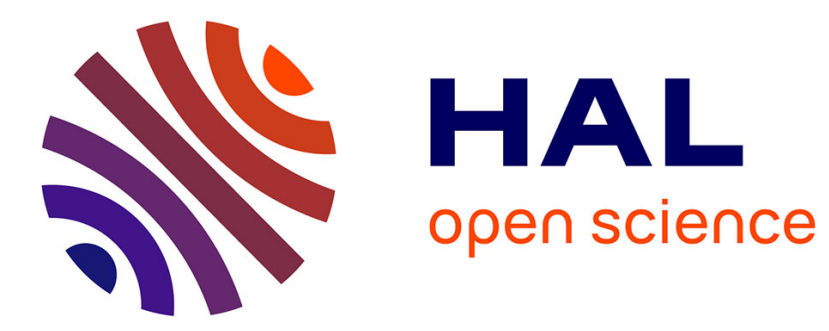

\title{
Pertinence et limitations de la loi de Mott dans les isolants désordonnés
}

\author{
F Ladieu, M. Sanquer
}

\section{To cite this version:}

F Ladieu, M. Sanquer. Pertinence et limitations de la loi de Mott dans les isolants désordonnés . Annales de Physique Colloque, 1996, 21, pp.267 - 336. 10.1051/anphys:199603001 . cea-01397319

\section{HAL Id: cea-01397319 https://hal-cea.archives-ouvertes.fr/cea-01397319}

Submitted on 15 Nov 2016

HAL is a multi-disciplinary open access archive for the deposit and dissemination of scientific research documents, whether they are published or not. The documents may come from teaching and research institutions in France or abroad, or from public or private research centers.
L'archive ouverte pluridisciplinaire HAL, est destinée au dépôt et à la diffusion de documents scientifiques de niveau recherche, publiés ou non, émanant des établissements d'enseignement et de recherche français ou étrangers, des laboratoires publics ou privés. 


\title{
Pertinence et limitations de la loi de Mott dans les isolants désordonnés
}

\author{
François Ladieu $\left({ }^{1}\right)$ et Marc Sanquer $\left({ }^{2}\right)$ \\ ( $\left.{ }^{1}\right)$ Laboratoire Pierre Süe (CEA-CNRS), DSM/DRECAM, CEA-Saclay, \\ 91191 Gif sur Yvette Cedex, France \\ $\left({ }^{2}\right)$ Service de Physique de l'État Condensé, DSM/DRECAM, CEA-Saclay, \\ 91191 Gif sur Yvette Cedex, France
}

Résumé. - Il y a déjà un quart de siècle que la loi de Mott a été établie afin de décrire le transport dans les milieux isolants désordonnés à basse température. Dans cet article de revue, nous rappelons brièvement les diverses étapes théoriques nécessaires à l'établissement rigoureux de la loi de Mott. Nous insistons sur le fait que la loi de Mott ne donne que la conductance moyenne d'un ensemble d'échantillons macroscopiques, tant que les interactions entre électrons restent négligeables. Nous étudions ensuite ce qu'il se passe lorsque l'on lève au moins l'une des hypothèses nécessaires à l'établissement de la loi de Mott. Nous nous penchons d'abord sur le cas des systèmes dont au moins une dimension n'est pas macroscopique : l'optimisation contenue dans la loi de Mott n'est alors plus représentative de la conductance mesurée. Enfin, nous essayons de rassembler des travaux relatifs aux effets des interactions entre électrons. S'il est acquis que, de façon générale, les interactions entraînent une divergence de la résistance plus rapide que ne le prédit la loi de Mott aux plus basses températures, la forme exacte de cette divergence ainsi que son interprétation restent encore sujets à caution. Nous rapprochons le travail d'Efros et Shklovskii et leur célèbre "gap de Coulomb" d'un travail plus récent sur les milieux granulaires dans lequel la taille des grains joue un rôle discriminant sur la nature de la divergence de la résistance à basse température. Nous suggérons que ce rapprochement ouvre une piste pour un modèle rendant compte de manière unifiée des diverses divergences mesurées à basse température pour la résistance électrique. Cette incursion dans les modèles granulaires nous permet aussi d'aborder la question des non linéarités en exposant les différences entre les prédictions du modèle des électrons chauds et celles proposées récemment pour un réseau de grains en dimension $d$.

\begin{abstract}
Twenty five years ago, Mott's law was established in order to describe electrical transport in disordered insulators at low temperature. In this review, we briefly summarize the different theoretical steps involved in the rigourous derivation of Mott's law. We stress upon the fact that Mott's law gives the mean conductance of an ensemble of macroscopic samples as long as electron-electron interactions remain negligible. We then study what happens when at least one of the key assumptions of Mott's law no longer holds. We first focus on systems whose size - at least in one dimension — is not macroscopic: the optimization involved
\end{abstract}

(C) Les Éditions de Physique 1996 
in Mott's law is no longer relevant for the measured conductance. Eventually, we try to gather different works dealing with electron-electron interactions. It is now established that interactions generally produce a stronger divergence for the electrical resistance than the one predicted by Mott's law at the lowest temperatures. But the exact shape of this divergence, as well as its interpretation, remain debated. We try to make a link between Efros and Shklovskii 's work and their famous "Coulomb gap" and a more recent work about granular media. In this latter work, the size of the grains is the key parameter for the shape of the divergence of the resistance at low temperature. We suggest this could indicate a way for a model accounting for the different shapes of divergence of the electrical resistance at the lowest temperatures. Furthermore this framework of granular media allows us to deal with non linear regime: we explain the main differences between the predictions of the hot electrons model and the ones recently derived for a $d$-dimensional network of grains.

\section{Table des matières}

1.1 Longueur de cohérence de phase en régime localisé . . . . . . . . 271

1.2 Résistance macroscopique en régime de saut de Mott . . . . . . . . 272

1.2.1 Expression détaillée des taux de transition associés aux sauts 272

1.2.2 Résolution du problème statistique par une approche de perco-

lation . . . . . . . . . . . . . . . . 274

1.3 Fluctuations de conductance dans les isolants macroscopiques . . . . 277

2 Effets de basse dimensionnalité

2.1 Conduction à quasi-1d en l'absence de fluctuations quantiques . . . . 280

2.1.1 Approche numérique percolative . . . . . . . . . . 281

2.1.2 Approches analytiques postulant l'optimisation locale . . . . 283

2.1.3 Comparaison entre optimisation locale et percolation . . . . . 290

2.2 Fluctuations quantiques à température finie : fluctuations quan-

tiques tronquées . . . . . . . . . . . . . . . . . 291

2.2 .1 Variations de température . . . . . . . . . . . . 291

2.2.2 Variations de niveau de Fermi (i.e. de tension de grille) . . . 293

2.2.3 Travaux expérimentaux et questions ouvertes . . . . . . . 296

2.3 Cas des systèmes courts et (très) larges . . . . . . . . . . . . . 297

2.3.1 Films de très grande section transverse . . . . . . . . . . 297

2.3.2 Films de section transverse finie $\left(S_{\perp}<S_{c}\right) \ldots \ldots . \ldots 301$

2.3.3 Confrontation aux travaux expérimentaux et questions ouvertes 303 
3 La si difficile question des interactions $\quad 306$

3.1 Interactions entre électrons et isolants de Mott . . . . . . . . . . . 306

3.1.1 La transition métal-isolant de Mott . . . . . . . . . . . . . 306

3.1.2 Blocage de Coulomb sur une seule île . . . . . . . . . . . . . 309

3.1.3 Réseau de grains capacitivement couplés . . . . . . . . . . . 313

3.2 Modèles homogènes : non linéarites et gap d'Efros et Shklovskii . . . 320

3.2.1 Modèles homogènes de non-linéarités . . . . . . . . . . . . . 320

3.2.2 Gap de Coulomb d'Efros et Shklovskii . . . . . . . . . . . . . . 324

3.2.3 Passage de la loi de Mott à celle d'Efros et Shklovskii . . . . 328

3.3 Unification possible de la loi d'Efros et Shklovskii $\gamma=\frac{1}{2}$ et des cas

à activation simple $\gamma=1$ ?. . . . . . . . . . . . . . . 329

4 Conclusion

Bibliographie

\section{Introduction}

L'application des principes de la mécanique quantique à la physique des solides a commencé il y a de nombreuses années et a permis des prédictions très importantes, observables même à des échelles grandes devant l'échelle atomique. Par exemple, la théorie des bandes pour les niveaux électroniques est maintenant couramment admise et est indispensable pour la conception de tous les composants intégrés dans les ordinateurs. Historiquement, la mécanique quantique a été d'abord introduite dans la théorie de l'état cristallin, car la symétrie par translation discrète permet de deviner la forme mathématique des fonctions d'onde. Ceci est le fameux théorème de Bloch, dont l'usage simplifie considérablement le traitement mathématique de l'équation de Schrödinger. En revanche, dans les systèmes désordonnés, la symétrie par translation discrète est perdue, ce qui rend bien plus difficile l'établissement de prédictions générales.

À la fin des années cinquante, Anderson fut le premier à proposer l'existence d'une transition métal-isolant lorsque la quantité de désordre est accrue dans un système [1]. Insistons tout d'abord sur le caractère particulier de cette transition. En effet, elle est liée à un changement des fonctions d'onde avec le désordre et non pas à une annulation de la densité d'états au niveau de Fermi. L'idée principale est que lorsque le désordre est faible les états propres électroniques restent délocalisés, alors que lorsque le désordre excède une certaine valeur critique les fonctions d'onde deviennent exponentiellement localisées autour d'une origine $r_{0}$ et sur une longueur de localisation $\xi: \Psi \propto \exp -\frac{r-r_{0}}{\xi}$. Cette localisation des états propres suggère l'existence d'une transition métal-isolant à température nulle $T=0$. Pour l'étude de cette transition il est important de faire le lien entre les propriétés de transmission de la surface de Fermi et la conductance d'un échantillon désordonné à $T=0$, lien établi par la célèbre formule de Landauer [2]. Soulignons que, en toute rigueur, la transition d'Anderson n'est définie qu'à $T=0$. C'est pourquoi 
l'étude expérimentale de cette transition requiert des températures les plus basses possibles, et pour fixer les idées disons que des traces observables de cette transition de température nulle subsistent jusqu'à $T \sim 1$ Kelvin.

Dans ce domaine du transport électrique dans les milieux désordonnés à basse température, le côté métallique de la transition d'Anderson a été étudié en bien plus grand détail que le régime isolant. En effet, tant que les états restent délocalisés, les méthodes perturbatives ainsi que les approches numériques peuvent être mises en place assez directement. De plus, en première approximation, la résistance électrique des métaux désordonnés sature à basse température. Pour des géométries usuelles, les résistances restent modérées même aux plus basses températures ce qui facilite leur mesure.

En revanche, beaucoup de questions demeurent ouvertes au sujet des isolants désordonnés. À $T=0$ plusieurs théories quantiques concurrentes existent, reposant sur des hypothèses différentes. Historiquement, la première approche du transport dans les isolants d'Anderson fut dérivée des modèles supersymétriques dus à Efetov et Larkin [3]. Ensuite, un modèle perturbatif dit "des chemins dirigés" fut proposé par Nguyen, Spivak et Shklovskii [4-6] et fut largement utilisé par les expérimentateurs en partie du fait de son caractère plus accessible que le modèle supersymétrique. Cependant, avec l'élaboration d'un modèle dérivé de la théorie des matrices aléatoires, Pichard et al. [7] firent éclater une polémique théorique, encore d'actualité aujourd'hui. En effet, la parution des travaux de Pichard et al. rendit évident le fait que les divers modèles de l'isolant d'Anderson mènent à des prédictions divergentes sur des questions aussi centrales que celle de l'évolution de la longueur de localisation $\xi$ avec le champ magnétique $H$ ou encore au sujet de l'influence du couplage spin orbite. Depuis, l'établissement d'un modèle semi classique dû à Bouchaud et Sornette [8] est venu raviver la polémique car ce dernier modèle aboutit à des prédictions qualitativement en accord avec celles de Pichard et al.. Nous ne détaillerons pas davantage le contenu de ce conflit théorique car son exposition exige un ouvrage entier et son dépassement demande que des progrès soient encore accomplis. Nous retiendrons seulement que l'approche théorique du transport quantique - à $T=0-$ dans les isolants d'Anderson est loin d'être unifiée.

Par ailleurs, les effets de température finie sur la résistance électrique $R$ sont extrêmement importants dans les isolants. C'est Mott qui a prédit [9], dix ans après le travail originel d'Anderson, que $R$ diverge de façon très spécifique dans les isolants désordonnés. L'argument de Mott peut se résumer très simplement: considérons deux états électroniques $i$ et $j$ respectivement localisés autour de $r_{i}$ et $r_{j}$ dans l'espace des positions. Désignons par $E_{i}$ et $E_{j}$ les énergies correspondantes et supposons par simplicité que $E_{i}<\mu<E_{j}$, où $\mu$ est le potentiel chimique. Mott considère la probabilité $P_{i, j}$ qu'un électron dans l'état $i$ se retrouve dans l'état $j$. Il écrit :

$$
P_{i, j} \propto \exp -\left(\frac{r_{i, j}}{\xi}+\frac{E_{i, j}}{T}\right),
$$

où la constante de Boltzmann est prise égale à $1, r_{i, j}=\left|r_{i}-r_{j}\right|$ et $E_{i, j}=E_{j}-E_{j}$. L'idée clef est que lorsque $r_{i, j}$ augmente les deux arguments de l'exponentielle 
varient en sens inverse. En effet, l'augmentation de $r_{i, j}$ décroît d'une part la probabilité que l'effet tunnel ait lieu — ce qui est exprimé par le fait que exp $-\frac{r_{i, j}}{\xi}$ décroît - ; mais d'autre part accroît la possibilité que $E_{i, j}$ soit petit. En effet, $E_{i, j}$ peut être choisi égal à l'écart entre niveaux dans une boite de taille $r_{i, j}$ contenant donc $\left(\frac{r_{i, j}}{\xi}\right)^{d}$ sites de localisation, en notant par $d$ la dimensionnalité du système. Si $T_{0}$ est l'écart de niveaux à l'échelle $\xi$, on obtient, en tirant les niveaux d'énergie indépendamment :

$$
E_{i, j}=T_{0}\left(\frac{r_{i, j}}{\xi}\right)^{-d}
$$

c'est-à-dire une diminution de $E_{i, j}$ lorsque $r_{i, j}$ augmente. L'utilisation de cette dernière expression dans $P_{i, j}$ permet de maximiser $P_{i, j}$ simplement en dérivant par rapport à $r_{i, j}$. Le saut le plus probable correspond à :

$$
r_{i, j}=r_{\mathrm{Mott}} \simeq \xi\left(\frac{T_{0}}{T}\right)^{\frac{1}{d+1}} \quad \text { et } \quad E_{i, j}=E_{\mathrm{Mott}} \simeq T\left(\frac{T_{0}}{T}\right)^{\frac{1}{d+1}}
$$

Ces deux quantités sont respectivement appelées "rayon de Mott" et "énergie de Mott" et définissent les paramètres du saut le plus probable à basse température. Notons que lorsque $T$ tend vers $0, r_{\text {Mott }}$ diverge et que $E_{\text {Mott }}$ tend vers 0 . Ceci reflète que dans les isolants désordonnés une optimisation peut être réalisée entre l'effet tunnel et le désordre en énergie. Ce mécanisme de conduction appelé saut à portée variable dominera à basse température car il produit une divergence extrêmement lente de la résistance électrique comparé à ce donnerait une activation simple du type loi d'Arrhénius. En effet, en supposant que $R \propto P_{i, j, \text { Mott }}^{-1}$, nous obtenons la fameuse prédiction de Mott :

$$
R \propto \exp \left(\frac{T_{0}}{T}\right)^{\frac{1}{d+1}}
$$

Cet article tente de faire le point sur la pertinence et les limites de cette loi de Mott. Dans un premier temps, nous résumons les différentes étapes théoriques nécessaires à l'établissement complet de la loi de Mott. Nous terminons cette partie en insistant sur la nature des hypothèses majeures mises en jeu ; à savoir les restrictions portant sur la dimensionnalité du système ainsi que l'absence supposée d'interactions entre électrons. Ceci nous permettra très naturellement d'aborder ensuite ce qu'il advient lorsque l'une au moins de ces hypothèses est fausse. Précisément, la seconde partie est consacrée aux systèmes de basse dimensionnalité, alors que la troisième tente d'aborder la difficile question des interactions. Soulignons que nous ne prétendons pas faire la liste des très nombreux travaux consacrés au transport par saut à portée variable. Nous préférons nous concentrer sur un petit nombre d'entre eux, afin de dresser un état de l'art de ce qui est compris et de ce qui l'est moins. 


\section{1. Établissement rigoureux de la loi de Mott}

Nous débutons cette section en définissant la longueur de cohérence de phase, c'està-dire l'échelle au delà de laquelle on peut additionner classiquement les probabilités dont on a vu qu'elles sont proportionnelles aux conductances locales. Ensuite, nous exposons (paragr. 1.2) minutieusement le travail d'Ambegaokar, Halperin et Langer [10] qui ont établi dans un article resté célèbre la loi d'évolution en température d'un échantillon macroscopique dont la conduction, au niveau mésoscopique, se fait par sauts à portée variable. Ayant établi ce que vaut la valeur moyenne de la résistance d'un échantillon macroscopique, nous nous intéressons (paragr. 1.3) à un travail de Pichard et Feng [11] calculant les fluctuations de conductance d'échantillon à échantillon lorsque le système est macroscopique mais de taille finie.

1.1. Longueur de COHÉREnCE DE PHASE en RÉgime LoCAlisé. - Dans l'introduction de cette revue, on vient d'expliquer que les interférences quantiques ainsi que les phonons jouent un rôle majeur pour le transport électrique dans les isolants désordonnés. En effet, la localisation d'Anderson est due aux interférences quantiques entre les diverses trajectoires de propagation d'un électron, interférences qui sont destructrices au delà de $\xi$ lorsque le désordre est fort. Par ailleurs les phonons jouent un rôle crucial pour la conduction puisque la loi de Mott est finalement l'optimisation entre tous les effets tunnels - assistés par phonon - possibles dans un système désordonné. Or il est très clair que l'irruption d'une perturbation extérieure à un système quantique brouille les interférences quantiques en ajoutant un terme de phase aléatoire à chaque trajectoire. Autrement dit, les phonons ont pour effet de supprimer le caractère quantique du transport au delà d'une échelle appelée "longueur de cohérence de phase" - ou encore longueur mésoscopique et notée $L_{\phi}$ : aux échelles inférieures à $L_{\phi}$ on aura à sommer de façon cohérente les amplitudes de probabilité, alors qu'aux échelles supérieures à $L_{\phi}$ une seule addition des probabilités suffira. La question qui se pose alors est de savoir ce que vaut $L_{\phi}$.

Il est tout d'abord évident que la longueur $L_{\phi}$ ne peut pas excéder $r_{\text {Mott. }}$. La question se pose ensuite de savoir si $L_{\phi}$ est égale à $r_{\text {Mott }}$ ou bien inférieure. À cet égard, on pourrait se demander si l'électron reçoit "son" phonon lorsqu'il est sur son site de départ ou bien sur son site d'arrivée. Un argument qualitatif simple serait alors de dire que l'électron est majoritairement sur son site de départ avant de sauter car sa probabilité de présence sur le site d'arrivée est exponentiellement faible. On serait alors tenté de dire que dans l'extrême majorité des cas la collision électron-phonon se produit sur le site de départ et donc que $L_{\phi}$ n'excède pas $\xi$.

En fait cet argument est erroné. On peut s'en rendre compte en se rappelant simplement que pour le saut entre deux sites séparés de la distance $r_{i, j}$ la probabilité de saut considérée par Mott est proportionnelle à $\exp \left(-\frac{r_{i, j}}{\xi}\right)$. Or ce terme est spécifiquement d'origine quantique. En effet, dans le cas d'une "particule" classique, comme par exemple la mise à l'équilibre d'une réaction chimique, la cinétique de transfert entre un état et un autre est bien thermiquement activée mais ne comporte pas de terme d'amortissement tunnel. Autrement dit, le saut de Mott est bien un 
effet quantique où l'électron se délocalise de manière cohérente sur le site d'arrivée alors qu'il est dans l'état quantique localisé sur le site de départ. Le saut de Mott est donc un effet quantique et de ce fait il est quantiquement cohérent. On conclut alors que $L_{\phi}=r_{\text {Mott }}$, le terme exp $\left(-\frac{r_{i, j}}{\xi}\right)$ désignant justement la somme cohérente des amplitudes de probabilités affectées à tous les chemins - y compris lorsqu'ils sont classiquement interdits - Il existe bien sûr une certaine probabilité que le transfert d'un site à l'autre se fasse de manière classique c'est-à-dire que l'électron soit excité dans un état de suffisamment haute énergie pour qu'il soit non localisé (i.e. métallique). Dans ce cas, le terme tunnel n'intervient pas dans la probabilité et le terme d'activation fait intervenir la différence entre l'énergie de Fermi $E_{\mathrm{F}}$ et celle du bord de mobilité $E_{\mathrm{M}}$ représentant l'énergie minimale des états non localisés. Cette différence $E_{\mathrm{M}}-E_{\mathrm{F}}$ est indépendante de la température contrairement à l'énergie de Mott $E_{\text {Mott }}$ qui décroît avec la température. C'est pourquoi la probabilité d'une transition "classique" sera tout à fait négligeable devant celle d'un saut de Mott à basse température.

1.2. Résistance macroscopique en RÉgime de saut de MotT. - L'argument de Mott que nous avons présenté en introduction ne donne que la résistance associée à un saut mésoscopique typique. Cependant, rien ne permet pour l'instant d'affirmer que cet argument donne le comportement de la résistance d'un échantillon macroscopique. En effet, il doit exister de nombreux cas où l'électron ne trouve pas de niveau éloigné de lui de $r_{\text {Mott }}, E_{\text {Mott }}$. Or la conductance $G_{i, j}$ entre deux sites $i$ et $j$ dépend exponentiellement de $r_{i, j}, E_{i, j}$. Il n'est donc pas évident de savoir dans quel sens les fluctuations du couple $r_{\text {Mott }}, E_{\text {Mott }}$ vont jouer. Va t-il y avoir compensation entre les sauts favorables $\left(G_{i, j}>G_{\text {Mott }}\right)$ et les sauts défavorables $\left(G_{i, j}<G_{\text {Mott }}\right)$ ? La réponse demande une analyse statistique fine qui fut réalisée pour la première fois par Ambegaokar, Halperin et Langer [10] dont nous détaillons ici le travail. Ceci permet d'établir une prédiction pour la résistance d'échantillons macroscopiques dont la conduction se fait par saut à portée variable à l'échelle mésoscopique. Signalons que, fort peu de temps après la publication du travail d'Ambegaokar et al., Pollak a publié indépendamment une étude sur le même sujet [12]. Le travail de Pollak conduit aux mêmes résultats principaux que celui d'Ambegaokar et al. mais utilise un formalisme moins simple ce qui lui permet d'aborder plus précisément des questions annexes qui sortent du cadre de cet article.

1.2.1. Expression détaillée des taux de transition associés aux sauts. - La première chose à faire est d'établir l'expression des taux de transition entre deux sites $i$ et $j$ séparés par la distance $r_{i, j}$. On notera $\Gamma_{i, j}$ le taux de transition de $i$ vers $j$ et $\Gamma_{j, i}$ le taux de transition de $j$ vers $i$. Notant par des crochets la moyenne thermique, on obtient :

$$
\Gamma_{i, j}=\left\langle n_{i}\left(1-n_{j}\right) \gamma_{i, j}\right\rangle
$$

où $n_{i}$ et $n_{j}$ sont les nombres de particules sur le site $i$ et $j$ et où $\gamma_{i, j}$ est un taux de transition intrinsèque indépendant de $n_{i}$ ainsi que de $n_{j}$ et comportant 
en particulier l'amortissement tunnel $\propto \exp -\frac{r_{i, j}}{\xi}$. ¿̀ l'équilibre thermique, $\left\langle n_{i}\right\rangle$ et $\left\langle n_{j}\right\rangle$ sont des grandeurs indépendantes statistiquement et l'on a donc :

$$
\left\langle n_{i} n_{j}\right\rangle=\left\langle n_{i}\right\rangle\left\langle n_{j}\right\rangle \text { où }\left\langle n_{i}\right\rangle=\frac{1}{1+\exp \frac{E_{i}}{T}}
$$

où $E_{i}$ est l'énergie - mesurée à partir du niveau de Fermi - du site localisé i. À l'équilibre thermique et en l'absence de champ électrique appliqué $(\mathcal{E}=0)$, on doit avoir $\Gamma_{i, j}=\Gamma_{j, i}$ afin d'assurer que le courant électrique est bien nul. Reprenant les deux expressions ci-dessus on obtient donc que nécessairement: $\gamma_{i, j}=\gamma_{j, i} \exp \frac{E_{i}-E_{j}}{T}$.

Il ne reste donc qu'à exprimer $\gamma_{i, j}$, puisque $\gamma_{j, i}$ s'en déduit immédiatement. La dépendance principale de $\gamma_{i, j}$ vis-à-vis de $r_{i, j}$ est évidente puisque l'on considère des transitions par effet tunnel $: \gamma_{i, j} \propto \exp -\frac{r_{i, j}}{\xi}$. La dépendance de $\gamma_{i, j}$ selon $E_{i}$ et $E_{j}$ est moins évidente. Les auteurs proposent qu'il existe une expression différente suivant que $E_{i}>E_{j}$ ou bien que $E_{i}<E_{j}$. En effet, dans le premier cas le transfert de $i$ vers $j$ se fait par émission de phonon alors que dans le second il se fait par absorption de phonon. Étant donné que les phonons sont rares à basse température, le second processus est plus rare que le premier d'un facteur $\exp -\frac{E_{j}-E_{i}}{T}$. Ceci revient à considérer qu'à basse température le terme d'émission spontanée de phonons est très supérieur aux termes d'émission ou d'absorption stimulées. C'est pourquoi, en première approximation, les auteurs proposent que :

$$
\gamma_{i, j}= \begin{cases}\gamma_{0} \exp -\left(\frac{r_{i, j}}{\xi}+\frac{E_{j}-E_{i}}{T}\right), & \text { si } E_{j}>E_{i} \\ \gamma_{0} \exp -\left(\frac{r_{i, j}}{\xi}\right) & \text { si } E_{j}<E_{i}\end{cases}
$$

où $\gamma_{0}$ est un taux de transition caractéristique du couplage électron phonon du matériau considéré. Combinant les équations précédentes, on obtient une expression assez simple pour $\Gamma_{i, j}^{0}$ où l'exposant 0 symbolise le fait que $\mathcal{E}=\mathbf{0}$ :

$$
\Gamma_{i, j}^{0}=\gamma_{0} \exp -\left[\frac{r_{i, j}}{\xi}+\frac{\left(\left|E_{i}\right|+\left|E_{j}\right|+\left|E_{i}-E_{j}\right|\right)}{2 T}\right]
$$

Lorsque $\mathcal{E} \neq \mathbf{0}$ un développement soigneux des taux de transition $\Gamma_{i, j}$ et $\Gamma_{j, i}$ permet d'écrire

$$
\Gamma_{i, j}(\mathcal{E})-\Gamma_{j, i}(\mathcal{E})=\frac{1}{T} \Gamma_{i, j}^{0}\left(e \mathcal{E}_{\mathbf{r}_{i, j}}+\delta \mu_{i}-\delta \mu_{j}\right)
$$

où $\delta \mu_{i}$ et $\delta \mu_{j}$ sont les variations du potentiel chimique aux sites $i$ et $j$ traduisant seulement les variations de $\left\langle n_{i}\right\rangle$ et $\left\langle n_{j}\right\rangle$ dues au champ $\mathcal{E}$. Notons que le terme entre parenthèses est la différence totale de potentiel entre les sites $i$ et $j$. C'est 
pourquoi, on peut interpréter la relation précédente en imaginant que les sites $i$ et $j$ sont reliés par une conductance $G_{i, j}$ valant:

$$
G_{i, j}=\frac{e^{2}}{T} \Gamma_{i, j}^{0}
$$

Retenons que la seule justification du taux de transition associé au saut à portée variable n'est pas si simple que nous l'avons présenté en introduction. Le problème consiste désormais à trouver la résistance globale d'un échantillon macroscopique dont chaque site est relié à tous les autres par une conductance $G_{i, j}$ donnée cidessus.

1.2.2. Résolution du problème statistique par une approche de percolation. Partant d'un réseau $d$-dimensionnel de sites tous reliés entre eux par les conductances $G_{i, j}$ que l'on vient d'exprimer, la question est désormais de savoir comment

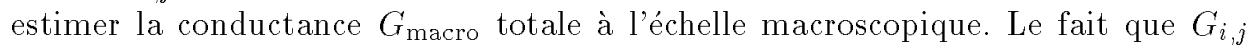
varie exponentiellement vite avec les paramètres du saut exclue que l'on se contente de prendre la valeur moyenne de ces paramètres pour obtenir $G_{\text {macro }}$. En effet, lorsque les fluctuations mésoscopiques sont fortes, la résistance totale d'un chemin de conduction n'est pas seulement déterminée par le nombre de résistances rencontrées - i.e. par sa longueur - mais aussi par la valeur des résistances les plus fortes rencontrées. Il va donc y avoir compétition entre des chemins "directs" - donc courts - mais comportant forcément quelques résistances très fortes, et des chemins très tortueux — donc longs - mais qui réussissent à éviter toute résistance trop forte. Cette optimisation se théorise au moyen de ce que l'on appelle génériquement "les modèles de percolation" et le travail d'Halperin et al. en est un exemple adapté au transport dans le régime de saut à portée variable.

Les auteurs commencent par classer tous les liens existant entre tous les sites par conductances décroissantes. Par la pensée, ils enlèvent tous ces liens et les rajoutent un par un en commençant par les plus conducteurs. À chaque étape, on teste pour savoir si il existe un chemin traversant l'échantillon. Dès qu'un tel chemin existe on stoppe le processus, car les liens que l'on pourrait rajouter ont une importance négligeable puisqu'ils se retrouvent en parallèle avec des liens exponentiellement moins résistants qu'eux.

La figure 1 illustre ce processus d'optimisation. Sur un réseau de $100 \times 100$ sites on a affecté une résistance $R_{i, j}$, entre sites premiers voisins, lognormalement distribuée, puisque $R_{i, j}=\exp \left(\eta_{i, j}\right)$ où l'on a pris les $\eta_{i, j}$ normalement distribués. Ensuite, on a (comme ci-dessus) enlevé toutes les résistances et on les a réintroduites une par une dans l'ordre des résistances croissantes. La procédure a été stoppée dès qu'un chemin traversant complètement l'échantillon s'est formé. Le chemin optimal obtenu est très tortueux, ainsi que nous l'avions qualitativement annoncé, et ceci est lié à la très large distribution des $R_{i, j}$. En effet, le chemin optimal correspondant aux $\eta_{i, j}$ a aussi été calculé et il se révèle beaucoup plus droit que celui optimisant $R$. Ces deux types de chemins correspondent à deux classes d'universalité étudiées dans ce que l'on appelle les problèmes “d'optimisation en milieu aléatoire" [14,15]. Nous n'entrerons pas davantage dans ce domaine, préférant recentrer notre propos 


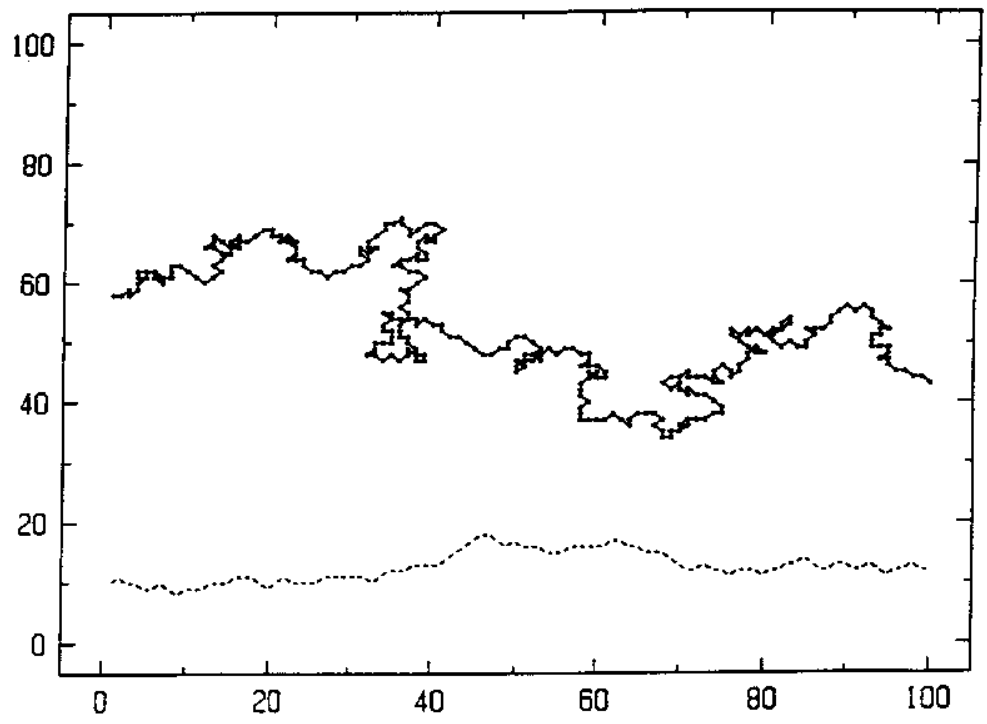

Fig. 1. - Percolation dans un réseau de $100 \times 100$ sites reliés chacun à leurs quatre premiers voisins. Le chemin tortueux est optimal si la résistance $R_{i j}$ reliant deux sites voisins est lognormalement distribuée, i.e. si $R_{i j}=\exp \eta_{i j}$ où les $\eta_{i j}$ sont normalement distribués. Même si sur cette figure le saut électronique est réduit aux premiers voisins, le chemin très tortueux est analogue à celui du transport en régime de saut à portée variable. Le chemin presque droit est optimal vis-à-vis de variables distribuées de façon étroite et dont on cherche à optimiser la somme le long d'un chemin traversant le réseau. Pour construire un tel chemin, on a donc posé $R_{i j}=\eta_{i j}$ où les $\eta_{i j}$ sont normalement distribués. Nous verrons l'utilité de ce chemin — qui est du même type que celui de la figure 14 au paragraphe 3.1.3.2. D’après [13].

[Percolation on a network containing $100 \times 100$ sites, each linked to its four nearest neighboors. Tortuous path is optimal if the resistance $R_{i j}$ between two neighboors is lognormally distributed, i.e. if $R_{i j}=\exp \eta_{i j}$ where the distribution of $\eta_{i j}$ is normal. Even if in this case the electronic hopping is restricted to the first neighboors, this tortuous path is similar to the one in variable range hopping transport. The nearly straight path is optimal when one has to optimize, along a path crossing the sample, the sum of random variables whose distribution is narrow. In order to construct such a path, when then set $R_{i j}=\eta_{i j}$ and take a normal distribution for $\eta$. We will show the relevance of this kind of path — which is the same than the one of figure 14 in the section 3.1.3.2. From [13].]

sur le cas où le saut à portée variable connecte tous les sites entre eux, et non pas seulement les premiers voisins comme sur la figure 1.

Revenant au travail d'Halperin et al. appelons $G_{c}$ la dernière valeur de conductance replacée dans le réseau au seuil de percolation. D’après ce que nous venons d'expliquer tout se passe comme si on pouvait oublier tous les liens tels 
que $G_{i, j}<G_{c}$. Vu l'expression de $G_{i, j}$, il s'agit donc de ne sélectionner que les liens pour lesquels:

$$
\eta_{i, j}=\frac{r_{i, j}}{\xi}+\frac{\left(\left|E_{i}\right|+\left|E_{j}\right|+\left|E_{i}-E_{j}\right|\right)}{2 T}<\ln \left(\frac{e^{2} \gamma_{0}}{T G_{c}}\right)
$$

Cette condition peut être sous dimensionnalisée en introduisant $R_{\max }=$ $\xi \ln \left(\frac{e^{2} \gamma_{0}}{T G_{c}}\right)$ et $E_{\max }=T \ln \left(\frac{e^{2} \gamma_{0}}{T G_{c}}\right)$ qui sont respectivement la longueur et l'amplitude en énergie maximales d'un saut électronique vu la condition $G_{i, j}>G_{c}$.

Tout le problème est désormais d'estimer $G_{C}$. Appelant, $n\left(E_{\mathrm{F}}\right)$ la densité d'états localisés par unité de volume et d'énergie au niveau de Fermi, on cherche le nombre $\mathcal{N}$ de sites par unité de volume tel que $\left|E_{i}\right|<E_{\max }$. On obtient, en supposant $n\left(E_{\mathrm{F}}\right)$ constante jusqu'à $E_{\max }$ :

$$
\mathcal{N}=2 E_{\max } n\left(E_{\mathrm{F}}\right)
$$

Pour obtenir $G_{c}$ on exige que dans un volume $R_{\max }^{d}$ il y ait au moins un lien qui satisfasse $G_{i, j}>G_{c}$. Autrement dit, on exige que :

$$
\mathcal{N} R_{\max }^{d}=\nu_{\mathrm{c}}
$$

où $\nu_{c}$ est d'ordre 1 et peut être plus précisément déterminée par l'étude de la percolation sur différents réseaux. Pour $d=3$, les auteurs estiment que $\nu_{c} \simeq 4$. Combinant les relations précédentes, on obtient :

$$
\ln \left(\frac{e^{2} \gamma_{0}}{T G_{\mathrm{C}}}\right)=\left(\frac{T_{0}}{T}\right)^{\frac{1}{d+1}} \text { où } T_{0}=\frac{4 \nu_{\mathrm{c}}}{n\left(E_{\mathrm{F}}\right) \xi^{d}}
$$

Notons que l'on retrouve la loi de Mott à l'échelle macroscopique mais que la valeur de $T_{0}$ est affectée d'un facteur $4 \nu_{c} \simeq 16$ provenant spécifiquement de la nature percolative du problème et que nous n'avions bien sûr pas obtenu par l'approche intuitive exposée en introduction. Signalons que, en toute rigueur, on ne peut pas directement faire appel aux résultats de percolation sur réseaux pour estimer $\nu_{c}$ car les liens du chemin de conduction ne sont pas tous de longueur égale, et l'on ne se trouve donc pas sur un réseau régulier. En effet, les valeurs de $r_{i, j}$ et de $E_{i}, E_{j}$ fluctuent de lien en lien et la seule contrainte que l'on impose pour construire le chemin de conduction est $G_{i, j}>G_{c}$. C'est pourquoi, il n'est pas surprenant que, suivant les auteurs, ce coefficient varie quelque peu : Efros et Shklovskii [16] donnent par exemple $4 \nu_{c} \simeq 21$ au lieu de 16. Expérimentalement, la loi de Mott a été observée dans de très nombreux systèmes, aussi bien dans des semi conducteurs cristallins dopés que dans des amorphes. Les références sont tellement nombreuses que nous préférons renvoyer au livre d'Efros et Shklovskii [16], contenant beaucoup de figures expérimentales.

\subsection{FluCtuations de CONDUCTANCE DANS les isolants maCRoscopiques}

Après avoir retrouvé la loi de Mott à l'échelle macroscopique, nous nous intéressons aux fluctuations d'échantillon à échantillon qui existent pour les systèmes macroscopiques de taille finie. L'idée est que la distribution des conductances est tellement 
large dans un isolant que les fluctuations mésoscopiques s'automoyennent très lentement au point que de telles fluctuations devraient être visibles dans des isolants millimétriques à très basse température. Nous nous focalisons sur un travail de Pichard et Feng [11] qui traite de cette question.

Les auteurs partent du point de vue d'un réseau de résistances aléatoires reliant a priori tous les sites de localisation entre eux. Les conductances du réseau sont notées $G_{i, j}=\exp -\eta_{i, j}$ et le fait qu'elles soient distribuées largement est exprimé par l'hypothèse que $\eta_{i, j}$ est "normalement" distribuée - d'après nos notations $\eta_{i, j}=\frac{r_{i, j}}{\xi}+\frac{E_{i, j}}{T}$ - Plus précisément, les auteurs supposent que $\eta_{i, j}$ est distribuée selon une distribution rectangulaire (par simplicité) de valeur non nulle entre $\eta_{1}$ et $\eta_{2}$. Afin de satisfaire à la loi de Mott les auteurs imposent :

$$
\langle\eta\rangle=\eta_{0}=\left(\frac{T_{0}}{T}\right)^{\frac{1}{d+1}}
$$

De plus, supposant la validité du résultat de matrices aléatoires, les auteurs imposent :

$$
\operatorname{var}(\eta)=\langle\eta\rangle=\eta_{0}
$$

ce qui exprime qu'à $T=0$ - où la longueur de cohérence de phase est la longueur $L$ de l'échantillon entier - les fluctuations de la transmission d'un système désordonné peuvent être traduites comme donnant lieu à une distribution gaussienne de $\frac{L}{\xi}$ dont la valeur moyenne et la variance sont égales [7]. La condition précédente sur la variance permet de déterminer immédiatement $\eta_{1}$ et $\eta_{2}$. On obtient $\eta_{1,2}=\eta_{0} \pm \sqrt{3 \eta_{0}}$. Il est facile de justifier que la forme rectangulaire de la distribution ne change pas qualitativement les prédictions - par rapport à ce que l'on obtiendrait avec une distribution gaussienne - lorsque la température est suffisamment basse pour que $\eta_{0} \gg 1$.

Les auteurs adoptent ensuite une approche de percolation assez similaire à celle exposée dans la section précédente. On commence par supprimer toutes les conductances inférieures à une conductance variationnelle $G_{\mathrm{v}}$ donnée. On calcule alors la conductance totale $G_{\text {res }}$ du réseau de conductances par le résultat de percolation stipulant que, pour une valeur donnée de $G_{\mathrm{V}}$ :

$$
G_{\mathrm{res}}=G_{\mathrm{v}}\left(p\left(G_{\mathrm{V}}\right)-p_{\mathrm{c}}\right)^{t_{\mathrm{p}}}
$$

où $p_{c}$ est le seuil de percolation et où $p\left(G_{\mathrm{v}}\right)=\int_{G_{\mathrm{v}}}^{G_{\max }} P(G) \mathrm{d} G$ en notant par $P(G)$ la distribution de probabilité de $G$. $t_{\mathrm{p}}$ est l'exposant de percolation caractéristique de $G$. On a $t_{\mathrm{p}}(d=2) \simeq 1,3$ et $t_{\mathrm{p}}(d=3) \simeq 1,9$. La relation donnant $G_{\text {res }}$ n'est bien sûr valable que si $p\left(G_{\mathrm{v}}\right)>p_{\mathrm{c}}$ puisqu'en dessous du seuil de percolation la conductivité du réseau est nulle. La détermination de $G_{\mathrm{v}}$ se fait par optimisation de $G_{\text {res }}$. En effet, plus $G_{\mathrm{V}}$ est petite et plus $p\left(G_{\mathrm{V}}\right)$ est grand ce qui augmente le second facteur donnant $G_{\text {res }}$ mais bien sûr diminue le premier. Puisque par construction on a toujours $G_{\mathrm{res}}<G$, la meilleure approximation de la conductance $G$ de 
l'échantillon sera donc donnée en fixant $G_{\mathrm{V}}$ de manière à maximiser $G_{\mathrm{res}}$. Du fait de la large distribution des conductances, l'écart entre la valeur optimale de $G_{\text {res }}$ et $G$ est petit.

Ce processus d'optimisation peut être réalisé analytiquement grâce aux hypothèses simplificatrices faites sur $P(\eta)$. On obtient que l'optimisation de $G_{\text {res }}$ correspond à une valeur de $p^{*}=p\left(G_{\mathrm{V}}\right)$ légèrement supérieure à $p_{\mathrm{c}}$ :

$$
p^{*}-p_{c} \simeq \frac{t_{\mathrm{p}}}{2 \sqrt{3 \eta_{0}}} \ll 1
$$

Par des arguments généraux de percolation, on peut établir l'existence de la longueur typique de percolation $\xi_{\mathrm{p}}$. $\xi_{\mathrm{p}}$ est la taille de ce que l'on appelle les "blobs", en d'autres termes c'est l'échelle au delà de laquelle le système redevient homogène. Concrètement, cette longueur diverge lorsque la température diminue, puisque alors les résistances sont de plus en plus largement distribuées - et donc de moins en moins automoyennantes - ce qui revient à dire qu'il faut explorer un volume de plus en plus vaste avant d'échantillonner suffisamment la distribution des $G_{i, j}$ pour avoir une grandeur représentative du comportement macroscopique. De manière équivalente, on peut dire que $\xi_{\mathrm{p}}$ diverge au seuil de percolation $p_{\mathrm{c}}$. Pour finir, on peut suggérer que, à l'intérieur d'un blob il existe au moins un chemin ne comportant pas de résistances locales trop fortes ; mais qu'à chaque extrémité de blob se trouve une résistance "dominante" c'est-à-dire à la fois forte et incontournable, dont la valeur influe notablement sur la résistance macroscopique. Découpant par la pensée le système en blobs dont la résistance est donnée, on obtient la résistance globale en additionnant les résistances de blobs en série et en parallèle, sans avoir à utiliser de relation d'échelle de percolation ni de dimension fractale. On peut montrer que si $r_{\mathrm{m}}$ est la longueur typique du saut de Mott, on a :

$$
\xi_{\mathrm{p}}=r_{\mathrm{m}}\left(p^{*}-p_{\mathrm{c}}\right)^{-\nu_{\mathrm{p}}} \quad \text { où } \quad \nu_{\mathrm{p}}(d=2)=\frac{4}{3} \text { et } \nu_{\mathrm{p}}(d=3)=0,9
$$

Soulignons que dans l'expression ci-dessus on retrouve bien une divergence de $\xi_{\mathrm{p}}$ lorsque l'on tend vers le seuil de percolation. Par ailleurs, les auteurs montrent que leurs hypothèses impliquent que, à l'échelle du blob, la conductance $G_{\text {blob }}$ est distribuée de sorte que son écart type égale sa valeur moyenne. Pour ce qui concerne la conductance globale, on déduit donc que les fluctuations seront réduites par un facteur égal à la racine carrée du nombre de blobs dans l'échantillon $\left({ }^{1}\right)$. Or, il y a $L^{d} \xi_{\mathrm{p}}^{-d}$ blobs dans l'échantillon. C'est pourquoi, les auteurs prédisent que la conductance $G$ d'un échantillon macroscopique de volume $L^{d}$ devrait fluctuer d'échantillon à échantillon d'une quantité $\delta G$ donnée par :

$$
\frac{\delta G}{\langle G\rangle}=\left(\frac{\xi_{\mathrm{p}}}{L}\right)^{d / 2}
$$

$\left({ }^{1}\right)$ On peut retrouver ce résultat en découpant l'échantillon en $\left(L \xi^{-1}\right)^{d-1}$ canaux en parallèle, i.e. en canaux de taille transverse $\xi_{\mathrm{p}}$. Vu que $\delta G_{\mathrm{blob}}=\left\langle G_{\mathrm{blob}}\right\rangle$ on obtient pour chaque canal $\frac{\delta R_{\text {canal }}}{\left\langle R_{\text {canal }}\right\rangle} \simeq \frac{\delta G_{\text {canal }}}{\left\langle G_{\text {canal }}\right\rangle}=\left(\frac{\xi_{\mathrm{p}}}{L}\right)^{1 / 2}$. Poursuivant le même raisonnement avec les $\left(L \xi^{-1}\right)^{d-1}$ canaux en parallèle on obtient le résultat annoncé. 
Le fait que dans la relation précédente ce soit $\xi_{\mathrm{p}}$ qui apparaisse et non pas $r_{\mathrm{m}}$ traduit que, à cause de sa distribution lognormale, la résistance ne s'automoyenne pas avant l'échelle $\xi_{\mathrm{p}}$, au delà de laquelle on peut employer le théorème de la limite centrale. Tout le point est qu'à basse température $\xi_{\mathrm{p}} \gg r_{\mathrm{m}}$ ce qui augmente notablement la fluctuation typique de la résistance globale. On peut cependant estimer que, avec les systèmes millimétriques, de telles fluctuations ne devraient pas être mesurables pour des températures supérieures à $100 \mathrm{mK}$. Les auteurs rappellent que l'approche de matrices aléatoires suggère que, dans les isolants désordonnés, les fluctuations de conductance sont ergodiques — c'est-à-dire qu'elles sont du même ordre lorsque l'on varie $H$ sur un échantillon ou bien lorsque on change le désordre microscopique - . Si cela est vrai, ces fluctuations de conductances dans des isolants macroscopiques pourraient expérimentalement être mesurées par simple variation du champ magnétique $H$ sur un échantillon donné lorsque la température est suffisamment basse, ce qui permettrait d'éviter d'effectuer une véritable moyenne d'ensemble sur un grand nombre d'échantillons microscopiquement différents. Ce genre d'expérience est cependant délicat car il exige à la fois des très basses températures - y compris en forts champs magnétiques — et en même temps des mesures d'isolants très résistants puisque leur résistance diverge à basse tempéra-

ture. À notre connaissance, seuls quelques auteurs ont enregistré des fluctuations de conductance dans des isolants macroscopiques [17].

Retenons de cette première partie que la loi de Mott donne le comportement de la résistance d'un isolant désordonné macroscopique à basse température si l'on suppose négligeables les interactions entre électrons, hypothèse que nous discuterons dans la dernière partie. Rappelons que la loi de Mott ne donne qu'une valeur moyenne de résistance macroscopique et qu'il subsiste des fluctuations de résistance entre échantillons tirés avec la même loi de distribution mais de configurations microscopiques différentes. Ces fluctuations s'atténuent extrêmement lentement à cause de l'apparition à basse température de $\xi_{\mathrm{p}} \gg r_{\text {Mott }}$, échelle au delà de laquelle seulement le système commence à s'automoyenner. En un sens on pourrait peut être dire que, de même que $L_{\phi}=r_{\text {Mott }}$ est l'échelle à partir de laquelle le système devient classique, de même $\xi_{\mathrm{p}}$ est l'échelle à partir de laquelle le système devient macroscopique. La divergence de $\xi_{\mathrm{p}}$ à basse température rend pertinente l'étude des cas où contrairement à ce que nous avons supposé jusqu'ici, le chemin optimal ne pourra plus se développer librement du fait de la taille du système. C'est ce que nous abordons dans cette deuxième partie intitulée "Effets de basse dimensionnalité".

\section{Effets de basse dimensionnalité}

Ainsi que nous venons de l'expliquer, la conduction à température finie dans un isolant d'Anderson se fait par sauts électroniques à l'échelle $r_{\text {Mott }}$. Dans les semiconducteurs dopés, pour $T=50 \mathrm{mK}, r_{\text {Mott }}$ atteint couramment la fraction de micron, ce qui est une échelle accessible avec les procédés modernes de lithographie. C'est $r_{\text {Mott }}$ qui permet commodément de définir la dimensionnalité du système : si les deux tailles transverses $L_{y}$ et $L_{z}$ sont inférieures à $r_{\text {Mott }}$ et que la longueur 
- dans le sens du courant - est grande devant $r_{\text {Mott }}$, on dira que l'on a un système quasi unidimensionnel, - le terme "quasi" rappelant que l'on ne travaille pas sur une chaîne d'atomes - . De même, si son épaisseur est inférieure à $r_{\text {Mott }}$ on qualifiera l'échantillon de bidimensionnel. À cause de la divergence de $r^{\prime}$ Mott à basse température, il est d'ailleurs possible qu'un système tridimensionnel à haute température devienne par exemple bidimensionnel à basse température ainsi que le suggèrent certaines expériences [18].

D'après ce que nous venons d'expliquer au paragraphe 1, la loi de Mott n'est valide que si le chemin de conduction très tortueux qualitativement décrit sur la figure 1 peut se développer librement au sein de l'échantillon. Il est évident que si les deux dimensions transverses $L_{y}$ et $L_{z}$ sont très inférieures à la taille de blob $\xi_{\mathrm{p}}$, le calcul de percolation justifiant la loi de Mott ne sera plus valable. C'est ce premier cas que nous commencerons par étudier en nous restreignant par souci de simplification aux systèmes quasi unidimensionnels : $L_{y} \simeq L_{z} \leq r_{\text {Mott }} \ll \xi_{\mathrm{p}}$ et $L_{x} \gg r_{\text {Mott }}$. Ceci nous permettra de dégager l'essentiel du comportement physique.

Pour la conduction dans les systèmes quasi $1 \mathrm{~d}$, nous procéderons en deux étapes. En premier lieu (paragr. 2.1), nous traiterons le problème en négligeant complètement l'existence de fluctuations quantiques dans le coefficient de transmission de chaque saut. Nous montrerons ensuite comment l'on peut réintroduire ces fluctuations (paragr. 2.2), et dans quelle mesure cette méthode est justifiée. Enfin (paragr. 2.3), nous aborderons le cas des systèmes courts $\left(L_{x}<\xi_{\mathrm{p}}\right)$ et de grande section transverse $L_{y} \gg r_{\text {Mott }}$ et/ou $L_{z} \gg r_{\text {Mott }}$. Nous montrerons, grâce à un travail de Raikh et Ruzin [19], que les propriétés de transport dans ces échantillons sont duales de celles des systèmes quasi $1 \mathrm{~d}$.

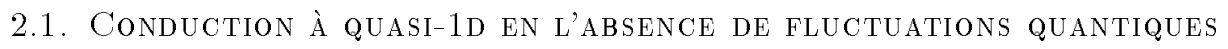

Ainsi que nous l'avons vu dans l'introduction de cette revue, l'argument le plus simple utilisé pour formuler la loi de Mott suppose que, partant d'un site de localisation, l'électron trouve au moins un site localisé éloigné de lui précisément de la distance $\left(r_{\mathrm{Mott}}, E_{\mathrm{Mott}}\right)$. Mais qu'arrive-t-il si, considérant un site de localisation, il n'existe pas de site de localisation correspondant au saut de Mott à la fois en position et en énergie? Si le système est bi ou tridimensionnel, le chemin de percolation fera le tour de la zone devenue hautement résistive du fait du manque de niveaux localisés de bonne énergie. Ceci est explicitement pris en compte par la théorie de percolation de Halperin et al. [10] et donne au chemin dominant la conduction un aspect tortueux. Cet aspect tortueux, en permettant d'éviter les régions pauvres en niveaux localisés à la bonne énergie, permet de retrouver que la résistance globale est sensiblement donnée par la résistance associée à un saut microscopique typique, à une redéfinition près de $T_{0}$.

Que se passe-t-il dans le cas quasi-1d qui nous intéresse ? Vu la contrainte topologique de cette situation, il est évident que le chemin ne peut pas faire le tour de la région très résistive. On s'attend donc à ce que la résistance globale soit plus 
divergente que la résistance de saut typique, i.e. on s'attend à avoir :

$$
R_{\text {globale }} \propto \exp \left(\frac{T_{0}}{T}\right)^{\gamma} \text { avec } \gamma>\frac{1}{2}=\gamma_{\text {Mott }}
$$

C'est Kurkijarvii [20] qui, dès 1973, a eu, le premier, l'idée que dans le cas quasi-1d, on obtenait $\gamma=1$, ce qui est le résultat correct dans la limite des fils extrêmement longs. Cependant, ce cas (des fils très longs) est le seul que l'approche des résistances effectives employée par Kurkijarvii puisse traiter. Or les situations expérimentales correspondent le plus souvent à des fils de longueur finie comme nous allons le voir. C'est pourquoi nous préférons détailler les travaux qui, à la suite du travail originel de Kurkijarvii mais selon des méthodes différentes, abordent le transport dans les systèmes quasi $1 \mathrm{~d}$ en couvrant à la fois le cas des fils très longs et celui des fils de longueur finie. Deux méthodes principales ont été utilisées pour le transport dans les isolants quasi 1d : d'une part des simulations numériques cherchant à optimiser la résistance globale du fil, d'autre part des approches analytiques postulant que le chemin de conduction peut être approximé en optimisant chaque résistance locale. Nous présentons chacune de ces deux approches dans les paragraphes 2.1.1 et 2.1.2 pour ensuite éclaircir le rapport qu'elles entretiennent (paragr. 2.1.3).

2.1.1. Approche numérique percolative. - En 1984 et 1985, Lee et al. [21,22] publiaient deux études numériques du problème, fondées sur une approche percolative que nous expliquons qualitativement. On commence par tirer aléatoirement des niveaux d'énergie correspondant aux différents sites de localisation qu'on a préalablement disposés sur une chaîne unidimensionnelle. On calcule ensuite les résistances électriques associées à tous les sauts possibles a priori entre états localisés selon la relation donnée au paragraphe 1.2 lorsque l'on a exposé le travail d'Ambegaokar, Halperin et Langer : ces résistances dépendant donc toutes fortement de la température. On enlève par la pensée tous les liens résistifs ainsi calculés et on les remet par ordre croissant de résistance. À chaque fois qu'on a remis une résistance, on teste pour savoir si il existe un chemin de conduction traversant l'échantillon. Dès qu'un tel chemin existe on arrête de rajouter des résistances et l'on approxime la résistance globale par la dernière résistance que l'on vient de rajouter. Ceci est le point clef de l'analyse : on arrête de rajouter des résistances car les résistances suivantes seraient exponentiellement plus fortes et donc ne dériveraient qu'une proportion infime de courant. On approxime la résistance globale avec la dernière résistance rajoutée parce que sa valeur est exponentiellement plus forte que la résistance rajoutée à l'étape précédente. Cette procédure traduit une idée importante : dans un fil quasi-1d, la distribution des résistances élémentaires est tellement large que la résistance d'un fil de longueur finie est dominée par une seule résistance de saut: celle qui est la plus résistante du meilleur chemin de conduction.

Par cette approche, on obtient, pour un échantillon et une température donnés, des fluctuations de la conductance fonction de l'énergie de Fermi ainsi que le montre la figure 2. Signalons que l'énergie de Fermi $E_{\mathrm{F}}$ est un paramètre contrôlable expérimentalement. En effet, les techniques de lithographie permettent de réaliser des 


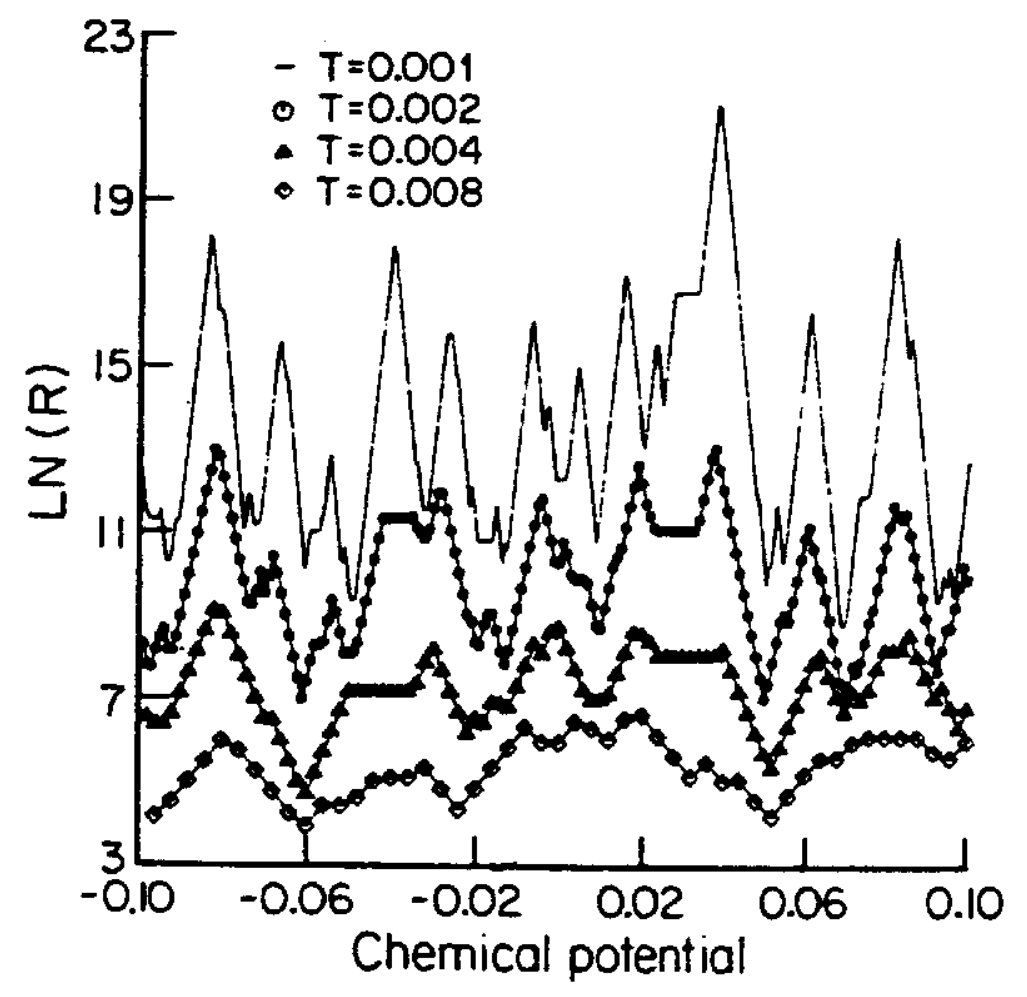

Fig. 2. - Fluctuations de $\ln G$ obtenues par Lee par simulation numérique du chemin de percolation dans un isolant quasi 1d désordonné. Les fluctuations deviennent géantes à basse température et sont dues seulement au changement de la résistance dominant le chemin optimal de percolation lorsque l'on varie le potentiel chimique $\mu$. D'après [21].

[Fluctuations of $\ln G$ obtained by Lee by numerical simulation of the percolating path in a quasi-1d disordered insulator. The fluctuations become large at very low temperature and are completely determined by the change of the dominant resistance along the percolating path when the chemical potentiel $\mu$ is varied. From [21].]

semiconducteurs dopés capacitivement couplés sur quelques microns à une grille métallique. En variant le potentiel électrique de la grille, on varie l'énergie potentielle des électrons dans le fil, ce qui varie par contrecoup leur énergie cinétique puisque leur énergie totale est fixée par le potentiel chimique imposé par les fils d'amenée du courant (ensemble grand canonique). La simulation numérique de la figure 2 est donc comparable aux expériences [23,24]. Notons que sur la figure 2 la dépendance en température de l'amplitude des fluctuations est très forte. Ceci a été très utilisé dans le débat théorique qui a opposé l'interprétation des fluctuations mesurées en termes de fluctuations quantiques du coefficient de transmission [25], 
et celle que prônent Lee et al. [21,26] où une simple variation de $E_{\mathrm{F}}$ suffit à modifier fortement la conductance locale calculée au paragraphe 1.2. Nous reviendrons sur cette question au paragraphe 2.2 .

En utilisant des arguments de percolation, les auteurs sont amenés, après de nombreuses approximations, à conjecturer que, si $T_{0}^{*}$ est l'écart typique en énergie entre niveaux voisins de $\xi$, alors en effectuant la moyenne sur le désordre :

$$
\langle\ln (R)\rangle=\eta_{0} \sqrt{\ln \left(\frac{2 L}{\xi}\right)} \text { en notant } \ln \left(R_{\mathrm{Mott}}\right) \text { par }: \eta_{0}=\sqrt{\frac{T_{0}^{*}}{T}}
$$

L'équation ci-dessus montre que même en moyenne on n'obtient jamais $R \propto L$, alors que l'approche la plus naïve aurait donné $R \propto \frac{L}{L_{\phi}}$. De plus la valeur de la pente de $\langle\ln (R)\rangle$ en fonction de $T^{-1 / 2}$ change avec la longueur $L$ du système. Soulignons que ce changement est très lent et que les auteurs ne font que le conjecturer. C'est pourquoi, ils testent cette relation sur des simulations d'un grand nombre de fils de longueurs différentes. Leur conclusion est que les résultats numériques sont en accord avec leur conjecture mais que la variation de $\sqrt{\ln \left(\frac{2 L}{\xi}\right)}$ dans leurs simulations est trop faible pour pouvoir établir la loi ci-dessus avec certitude. Cette dépendance inhabituelle de $\langle\ln (R)\rangle$ avec $L$ est reliée à ce que nous exposerons plus loin : dans un fil quasi-1d dans le régime de Mott, la résistance globale est dominée par la plus forte résistance microscopique rencontrée. Plus le fil est long et plus cette résistance est forte, d'où la dépendance non triviale en taille dans l'expression ci-dessus.

2.1.2. Approches analytiques postulant l'optimisation locale. - Nous entendons par "approche locale", un calcul dont l'objectif soit de répondre à la question suivante : quelle est la probabilité qu'une région pauvre en niveaux localisés proche de $E_{\mathrm{F}}$ apparaisse et engendre donc une résistance forte de valeur donnée ? La réponse à une telle question permet d'approximer la résistance de l'échantillon étudié. En effet, pour un fil comportant $N$ sauts électroniques, on peut alors trouver la résistance $R_{\max }$ dont la valeur d'occurrence est $1 / N$. Cette résistance donne une approximation de la résistance totale du fil considéré, car les résistances supérieures sont trop rares pour avoir un poids et les résistances plus faibles, bien que fréquentes, sont exponentiellement moindres que $R_{\max }$ ce qui fait qu'elles affectent peu la résistance totale. Raikh et Ruzin [27] ont été les premiers à utiliser cette démarche pour le problème du transport dans les isolants quasi 1d. Notons que cette méthode est locale au sens où elle n'exprime pas que cette résistance forte $R_{\max }$, dont on calcule la fréquence d'occurrence, appartienne au chemin de percolation, i.e. au meilleur chemin de conduction. A priori, cette approche locale est déconnectée de l'approche globale des calculs de percolation [21,22]. Afin d'étudier dans quelle mesure ces deux méthodes différentes convergent vers des prédictions identiques, nous exposons le travail de Ladieu et Bouchaud [28]. Ce travail reprend la démarche de Raikh et Ruzin mais en exprimant de surcroît les corrélations entre 
deux résistances consécutives le long d'un chemin de conduction. En effet, comme le montre l'équation ci-dessous, les valeurs de deux résistances consécutives, le long d'un chemin de conduction, ne sont pas totalement indépendantes car elles mettent toutes les deux en jeu les coordonnées du site intermédiaire. Le travail que nous exposons maintenant tient compte de la corrélation entre deux résistances consécutives et construit le meilleur chemin possible en optimisant la résistance à chaque saut. Nous verrons ensuite (paragr. 2.2.3) en quelle mesure cette approche locale converge vers les résultats percolatifs de Lee et al.

2.1.2.1. Équation auto-cohérente postulant l'optimisation locale. - Afin de résoudre le problème de Mott quasi-1d, supposons les niveaux d'énergie localisés indépendants entre eux et distribués au hasard avec une densité d'états $\rho$ par unité de longueur et d'énergie. La résistance associée au saut entre 2 sites $\left(x_{i}, E_{i}\right)$ et $\left(x_{j}, E_{j}\right)$ est donnée - d'après ce que nous avons vu au paragraphe 1.2 - par :

$$
R_{i j}=R^{*} \exp \left[\frac{\left|E_{i}\right|+\left|E_{j}\right|+\left|E_{i}-E_{j}\right|}{2 T}+\frac{\left|x_{i}-x_{j}\right|}{\xi}\right]
$$

où $R^{*}$ est une résistance typique (métallique) à l'échelle $\xi-i . e$. on peut prendre $R^{*} \simeq R_{Q}=\frac{h}{e^{2}}$ - On omettra de mentionner $R^{*}$ i.e. on la posera égale à 1 . Par convention dans la formule ci-dessus $E=0$ correspond au niveau de Fermi. Soulignons que dans l'équation ci-dessus nous prenons $\xi=$ Constante, négligeant complètement les fluctuations d'origine quantique que l'on peut interpréter comme donnant à $\xi^{-1}$ une distribution gaussienne [7]. Ces fluctuations seront prises en compte au paragraphe 2.2 .

À partir de l'équation précédente, le problème est donc de déterminer la résistance totale entre $(x=0, E=0)$ et $(x=L, E=0)$ d'un échantillon donné, connaissant la distribution des $E_{i}$. Même si le problème est uni-dimensionnel, ceci n'est pas simple car chaque site est relié à tous les autres sites. Comme cela a déjà été fait pour ce problème [10], du fait de la variation exponentiellement rapide de $R_{i j}$, on approxime la résistance globale à l'échelle $L$ par celle du chemin le moins résistant. De plus, l'équation donnant $R_{i j}$ montre que ce chemin optimal ne peut pas s'éloigner arbitrairement loin du niveau de Fermi. On fait donc l'hypothèse que le meilleur chemin peut être construit en choisissant toujours localement le lien le moins résistant. On montrera au paragraphe 2.1.3. que cette hypothèse mène asymptotiquement au bon résultat. De toute façon par cette méthode nous obtenons une borne supérieure pour la résistance.

Soit $P(\eta \mid m)$ la probabilité qu'à un point donné du chemin optimal, l'électron effectue un saut de résistance $R \equiv e^{\eta}$, en étant parti d'un site d'énergie $E \equiv$ $m T$. Afin de calculer $P(\eta \mid m)$, demandons nous d'abord : quelle est la probabilité $\int_{\eta}^{\infty} \mathrm{d} \eta P(\eta \mid m)$ que le lien le moins résistant, à partir de ce site d'énergie $E \equiv m T$, ait une résistance supérieure ou égale à $R \equiv e^{\eta}$ ? Pour que ceci arrive, on doit avoir une région pauvre en niveau localisé, i.e. aucun niveau ne doit se trouver à l'intérieur de l'aire hachurée de la figure 3 . Ceci arrive $\left({ }^{2}\right)$ avec une probabilité

$\left({ }^{2}\right)$ Nous négligeons les corrélations de Wigner entre les différents niveaux d'énergie, ce 


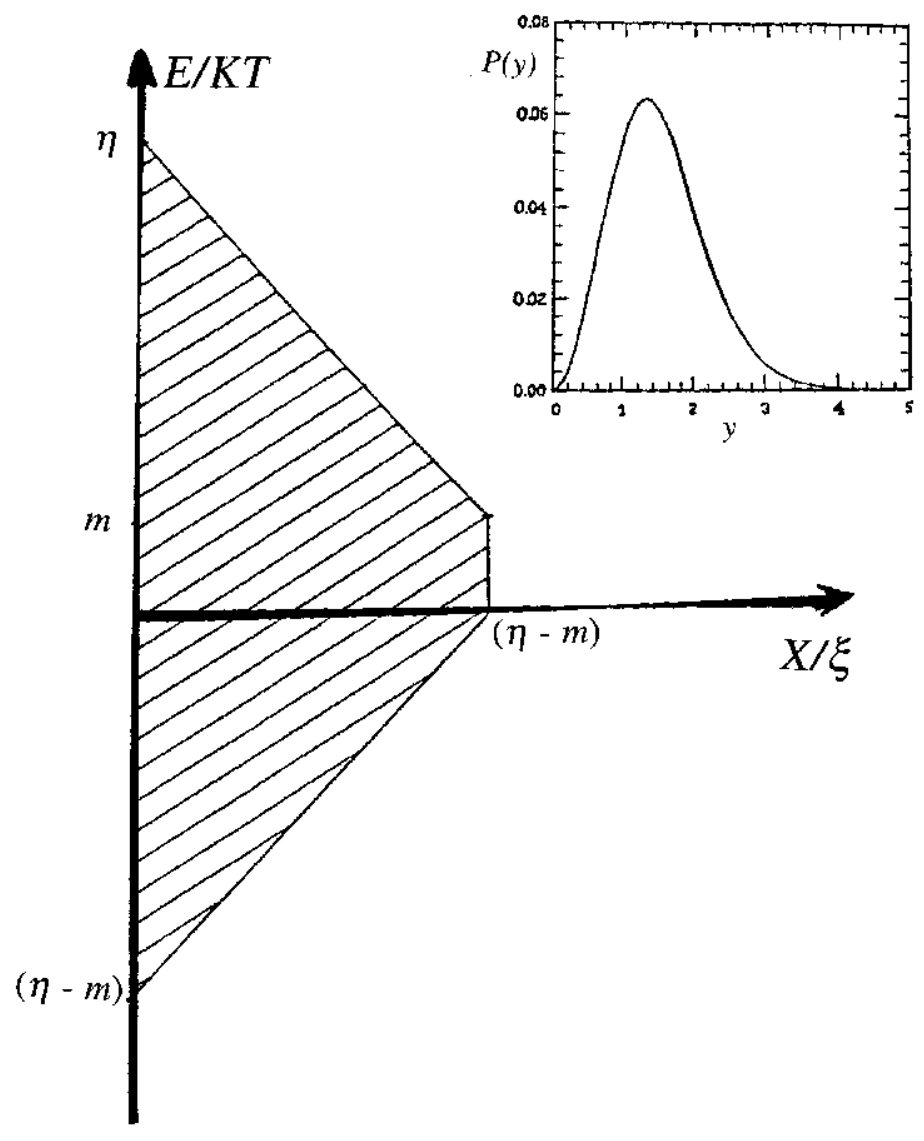

Fig. 3. - Lorsqu'un électron part du site $x=0, E=m T$, aucun site localisé ne doit se trouver dans la région hachurée du plan $(x, E)$ pour que la résistance de saut soit $\geq e^{\eta}$. En encart : détermination numérique de $P(y)$ en fonction de $y=\frac{\eta}{\eta_{0}}$. La valeur la plus probable de $y$ est 1,3 , la valeur moyenne de $y$ est 1,5 . D'après [28].

[Starting from the site $x=0, E=m T$, no localised sites must be present in the hatched region of the $(x, E)$ plane for the resistance to be larger than $e^{n}$. Insert: Numerical determination of $P(y)$ versus $y=\frac{\eta}{\eta_{0}}$. The most probable value of $y$ is 1.3 , the mean value of $y$ is 1.5 . From [28].]

$\propto \exp \left[-\frac{\eta(\eta-m)}{\eta_{0}^{2}}\right]$ où $\eta_{0}^{2} \equiv \frac{1}{\rho T}$, i.e. où exp $\eta_{0}$ est simplement la résistance mésoscopique associée à un saut de Mott typique et $r_{0} \equiv \eta_{0} \frac{\xi}{2}$ est la taille du saut de Mott typique.

qui est justifié dès que $e^{\eta} \gg \eta_{0}^{2},[29]$. 
Nous allons supposer que $r_{0} \ll L$, ce qui nous permet de négliger les effets "tunnel-résonnants", i.e. les cas où la mise en série de deux résistances élémentaires peut donner une résistance globale très inférieure à la somme des deux résistances élémentaires. Négligeant statistiquement cette possibilité grâce à notre hypothèse $r_{0} \ll L$, il est possible, par simple dérivation de la probabilité précédente par rapport à $\eta$, d'exprimer la probabilité totale $P(\eta)$ qu'une résistance $e^{\eta}$ appartienne au chemin optimal :

$$
P(\eta)=\int_{0}^{\eta} \mathrm{d} m P(\eta \mid m) \mathcal{Q}(m)=\int_{0}^{\eta} \frac{\mathrm{d} m(2 \eta-m)}{\eta_{0}^{2}} \exp \left[-\frac{\eta(\eta-m)}{\eta_{0}^{2}}\right] \mathcal{Q}(m)
$$

où l'on note par $\mathcal{Q}(m)$ la probabilité de trouver l'électron à l'énergie $m T$ le long du meilleur chemin de conduction. Soulignons que cette probabilité, relative à un phénomène hors équilibre, n'est pas donnée par le poids de Boltzmann.

On peut obtenir une équation de fermeture pour $P(\eta)$ en remarquant que puisque $\eta$ peut uniquement augmenter lorsque $m$ augmente, on a :

$$
\int_{\eta}^{\infty} \mathrm{d} \eta^{\prime} P\left(\eta^{\prime} \mid m\right)=\int_{0}^{m} \mathrm{~d} m^{\prime} P\left(m^{\prime} \mid \eta\right)
$$

Dans la relation précédente, $P(m \mid \eta)$ est la probabilité que l'on trouve l'électron à l'énergie $m T$ alors qu'il vient de faire un saut de résistance $e^{\eta}$. Remplaçant cette dernière égalité dans la précédente, il est possible d' éliminer $\mathcal{Q}(m)$ et une équation auto-cohérente permettant de calculer $P(\eta)$ :

$$
P(\eta)=\int_{0}^{\eta} \frac{\mathrm{d} m(2 \eta-m)}{\eta_{0}^{2}} \exp \left[-\frac{\eta(\eta-m)}{\eta_{0}^{2}}\right] \int_{m}^{\infty} \frac{\mathrm{d} \eta^{\prime} \eta^{\prime}}{\eta_{0}^{2}} \exp \left[-\frac{\eta^{\prime}\left(\eta^{\prime}-m\right)}{\eta_{0}^{2}}\right] P\left(\eta^{\prime}\right)
$$

Cette équation a été résolue numériquement [28] : sur la figure $3, P$ est porté en fonction de la variable $y=\frac{\eta}{\eta_{0}}$, avec, rappelons le, $\eta_{0}=\ln \left(R_{\mathrm{Mot}}\right)=\left(\frac{T_{0}^{*}}{T}\right)^{1 / 2}$. On peut montrer en particulier que $P(y) \simeq y^{2}$ à petit $y$ et que $P(y) \simeq 2 \exp -\frac{y^{2}}{2}$ lorsque $y$ est grand. Soulignons que, du fait de l'expression auto-cohérente de $P(\eta)$ et de $\mathcal{Q}(m)$, les corrélations existant entre les résistances appartenant au chemin optimal sont explicitement prises en compte. La comparaison avec les résultats de Raikh et Ruzin - établis en négligeant ces corrélations — devrait donc renseigner sur leur importance.

Enfin, avant d'aller plus loin dans notre analyse, notons que l'hypothèse de négliger les fluctuations quantiques n'est valable que lorsque la largeur $w_{q}$ de leur distribution est petite devant celle $w_{\text {geo }}$. de la distribution due à la répartition géométrique des niveaux. D'après l'équation auto-cohérente, on obtient $w_{\text {geo }} \simeq \eta_{0}$, alors que l'on peut estimer que $w_{q} \simeq \eta_{0}^{\omega}$, avec $\omega=\frac{1}{2}$ selon la théorie des matrices aléatoires [7], et $\omega=\frac{1}{5}$ selon celle des chemins dirigés [5]. C'est pourquoi cette approche ne sera pas valable lorsque $\eta_{0} \simeq 1$, i.e. trop près de la transition métal-isolant. 
2.1.2.2. Calcul des deux premiers moments de la résistance globale. - La résistance globale d'un système de taille $L$ est donné par $R=\sum_{i=0}^{N} R_{i}$, où $N=\frac{L}{r_{0}}=\frac{2 L}{\eta_{0} \xi}$ est le nombre total de sauts, et où $\ln \left(R_{i}\right)$ sont distribuées selon $P$. Signalons trois points importants dans les quelques lignes précédentes :

- les tailles des sauts de Mott du chemin optimal sont distribuées de façon étroite. En effet, comme le montre la figure 3, la distribution de $\eta$ est étroite, i.e. dont les tirages s'automoyennent rapidement. Or, puisque $\eta_{i, j}=\frac{r_{i, j}}{\xi}+$ $\frac{E_{i, j}}{T}$, la distribution de $\eta$ est a priori plus large que celle de $\frac{r_{i, j}}{\xi}$. Puisque, par hypothèse, on a jusqu'ici négligé les fluctuations de $\xi$, on en déduit donc que les $r_{i, j}$ sont distribués de façon étroite. C'est pourquoi, on utilise $r_{0}$ pour calculer $N$.

- le fait d'additionner simplement les résistances $R_{i}$ est possible grâce à l'hypothèse $r_{0} \ll L$, ce qui permet de négliger statistiquement les effets tunnels résonnants.

- les résistances $R_{i}$ sont, elles, très largement distribuées et le théorème de la limite centrale n'est pas simplement applicable car lorsque $N$ n'est pas très grand, la somme de $N$ résistances tirées selon $P$ ne suit pas un comportement moyen. C'est ce que nous discutons maintenant.

\section{Cas des fils très longs}

Nous entendons par "fils très longs" que le théorème de la limite centrale est applicable, réservant à plus tard de quantifier cette condition vis-à-vis de $N$. Dans ce cas on a donc

$$
\langle R(N)\rangle=N \int_{0}^{\infty} \mathrm{d} \eta P(\eta) e^{\eta}
$$

Puisque dans ce cas les résistances sont des variables auto-moyennantes, on calcule directement $\langle R\rangle$ et l'on trouve :

$$
\ln \left(\frac{\langle R\rangle}{N}\right) \simeq 1,5 \eta_{0} \quad \text { pour } \quad \eta_{0}=\mathcal{O}(1)
$$

i.e. très proche de la transition nous retrouvons la loi de Mott. En revanche, du fait de la lente décroissance de $P$, il vient :

$$
\ln \left(\frac{\langle R\rangle}{N}\right) \simeq \frac{\eta_{0}^{2}}{2} \propto T^{-1} \quad \text { pour } \quad \eta_{0} \gg 1
$$

Ceci signifie que, si l'on est pas très proche de la transition métal-isolant, la loi de Mott n'est pas suivie à quasi-1d, même dans les fils très longs. Ceci avait déjà été trouvé par Kurkijarvii [20], ainsi que par Raikh et Ruzin [27]. Soulignons que 
la valeur de la résistance obtenue est à peu près la plus forte résistance que l'on puisse obtenir dans un fil, pour lequel $T$ et $T_{0}$ sont donnés. En effet, deux sites voisins de $\xi$ ont un écart entre leurs niveaux d'énergie inférieur ou égal à $T_{0}^{*}$. La

résistance qui les relie ne peut donc dépasser $e^{\frac{T_{0}^{*}}{T}+1}$ i.e. $e^{\eta_{0}^{2}+1}$. Ces sauts les plus résistifs possibles résistifs sont inévitables dans des fils très longs. Puisque ce sont eux qui dominent la résistance, il n'est pas surprenant que le résultat de cette approche tenant compte des corrélations soit en accord avec ceux des modèles les négligeant [20,27]. En effet pour ces sauts les plus résistants possibles l'existence de corrélations est évidement sans importance.

\section{Fils de longueur finie}

Notons tout d'abord que la valeur maximale $\eta_{\max }$ obtenue lorsque l'on effectue $N$ tirages $\left(^{3}\right)$ selon la distribution $P$ est typiquement d'ordre $\eta_{\max } \simeq \eta_{0} \sqrt{2 \ln (a N)}$ où $a \simeq 2$. Le théorème de la limite centrale ne s'applique certainement pas tant que $\eta_{\max }<\ln \left(\frac{\langle R\rangle}{N}\right)$ puisque cela amènerait la situation absurde où la moyenne est plus forte que le plus grand élément du tirage. Dans un tel cas, la résistance globale à l'échelle $L$ est dominée par le lien le plus résistif, i.e. par $\eta_{\max }$. On peut comprendre aussi cela en notant que pour les valeurs intermédiaires de $R \equiv e^{\eta}$, la distribution $P(R)$ se comporte comme une loi de puissance :

$$
P(R)=R^{-(1+\mu)} \text { où l'exposant effectif vaut } \mu=\frac{\ln (R)}{2 \eta_{0}^{2}}
$$

Or pour $\mu<1$, la somme donnant $R$ est une "somme de Lévy" dont on sait qu'elle est dominée par ses quelques plus grands termes [30]. ¿ partir de ces résultats, on constate que la condition $\mu\left(\eta_{\max }\right) \simeq 1$ équivaut à $\eta_{\max } \simeq 2 n_{0}^{2}$. C'est cette condition que l'on peut prendre pour séparer le cas des "très longs fils" de celui des fils de longueur finie. Utilisant le lien entre $\eta_{\max }$ et $N$, on dira donc que l'on est dans le cas des fils de longueur finie lorsque:

$$
N<N^{*} \simeq \frac{1}{a} \exp \left(2 \eta_{0}^{2}\right) \text { où } a \simeq 2
$$

Concrètement $N^{*}$ représente le nombre minimal de sauts de Mott qu'il faut avoir en série pour que la résistance macroscopique ne soit plus dominée par une seule résistance mésoscopique dominante. Assez fréquemment, le domaine expérimental correspond le plus souvent au cas $N<N^{*}$ ainsi que le domaine exploré par les simulations numériques [21]. C'est pourquoi, il est important de calculer précisément les deux premiers moments de la résistance dans ce cas. Puisque dans ce cas, $\langle R\rangle$ est dominée par des événements rares, on s'intéresse à $\langle\ln (R)\rangle$. En effet, la variable $\ln (R)$ est, selon ce qui précède, distribuée de façon étroite, i.e. sa valeur typique est proche de sa valeur moyenne. Or, si l'on désire comparer l'analyse théorique à

$\left({ }^{3}\right)$ à condition que $N$ ne soit pas trop proche de 1. 
l'expérience (numérique ou physique), il est nécessaire de choisir une variable distribuée de façon étroite. Sinon, la confrontation théorie/expérience demande qu'un très grand nombre d'échantillons soient testés, ce qui n'est en pratique jamais possible. Nous avons vu que la résistance de l'échantillon est donnée par le saut le plus résistif, i.e. $\ln (R) \simeq \eta_{\max }$. Vu la valeur typique de $\eta_{\max }$, on obtient donc :

$$
\langle\ln (R)\rangle \simeq \eta_{0} \sqrt{2 \ln (a N)}
$$

Dans le cas des fils de longueur finie, on conclut donc que $\langle\ln (R)\rangle$ se comporte comme $T^{-1 / 2}$, i.e. ainsi que la loi de Mott le prédirait sauf que la pente de $\langle\ln (R)\rangle$ fonction de $T^{-1 / 2}$ augmente avec $N$ comme $\sqrt{2 \ln (a N)}$.

Passons à l'amplitude typique des fluctuations : leur ordre de grandeur est gouverné par la largeur de la distribution de $\eta_{\max }$, dont on peut montrer, en utilisant la décroissance asymptotique de $P$, qu'elle est d'ordre $\simeq \frac{\eta_{n}^{2}}{\eta_{\max }}$. On trouve donc :

$$
\frac{\Delta \ln (R)}{\langle\ln (R)\rangle} \simeq \frac{1}{2 \ln (a N)}
$$

Comment ces deux prédictions se comparent-elles à ce qui a été établi dans les travaux précédents ? Raikh et Ruzin par leur approche analytique fondée sur l'optimisation locale négligeant les corrélations ont calculé la distribution complète de $\ln (R)$. Une comparaison soigneuse de ces résultats aux leurres ne révèle qu'une petite différence : alors que ici, $a$ est une constante $(a \simeq 2)$, pour Raikh et Ruzin $a=\sqrt{\ln \left(\frac{L}{\xi}\right)}$. Qualitativement, cela signifie que pour une longueur $L$ donnée, le fait de négliger les corrélations augmente la valeur prédite pour $\langle\ln (R)\rangle$. En d'autres termes, l'expression auto-cohérente de $\mathcal{Q}(m)$ renforce l'importance des sauts entre niveaux proches du niveau de Fermi, ce qui amène naturellement une diminution de la résistance globale moyenne. Cependant, cet effet est trop petit pour être testéque ce soit numériquement ou expérimentalement - puisqu'il ne croît que comme $\ln (\ln L)$.

2.1.3. Comparaison entre optimisation locale et percolation. - Comme on l'a déjà dit, Lee utilise une méthode percolative pour trouver la résistance globale, i.e. il n'utilise pas d'hypothèse d'optimisation locale. Pourtant, les expressions que nous venons d'établir — en utilisant l'optimisation locale — pour les deux premiers moments de $\ln (R)$ sont en excellent accord avec les résultats de percolation [21] et $[22]$.

Pour mettre en évidence les différences entre les prédictions de Lee et ceux exposés au paragraphe 2.1.2 une comparaison très détaillée doit être menée à bien. Pour cela, il faut réaliser des simulations numériques analogues à celles de Lee mais en utilisant l'hypothèse d'optimisation locale au lieu d'une approche percolative. Deux différences principales sont notables: 
- En premier lieu, l'approche de percolation donne des valeurs de $\langle\ln (R)\rangle$ légèrement plus faibles que celles prédites par l'équation précédente $\left({ }^{4}\right)$ : ceci n'est pas surprenant car a priori la meilleure optimisation possible s'obtient par méthode percolative. Cependant, cette différence tend vers zéro lorsque $N$ croît. Pour le système le plus court à la plus basse température $(N=12)$ la différence entre les $\langle\ln (R)\rangle$ est de $20 \%$, alors que pour la plus forte température et le système le plus long $(N=143)$ cette différence tombe à $12 \%$.

- En second lieu, les fluctuations de conductance que Ladieu et Bouchaud obtiennent en faisant varier $E_{\mathrm{F}}$ dans leurs simulations sont souvent dissymétriques ainsi que le révèle un simple coup d'œil à la figure 4 : certaines fluctuations ont des pentes verticales sur un côté et pas sur l'autre. Il a été numériquement vérifié que en ces points précis la procédure d'optimisation locale est en défaut, i.e. le meilleur chemin ne peut pas être obtenu en optimisant à chaque saut de Mott. Cependant ceci change fort peu la taille globale des fluctuations. Pour $N \simeq 50$, ce qui sera un ordre de grandeur expérimental typique [23,24], on a moins de $10 \%$ de différence entre l'amplitude de ces fluctuations et celle qu'obtient Lee par simulation de percolation. De plus, à grands $N$ cette différence tend vers zéro.

Nous concluons, d'après cette comparaison détaillée, que l'hypothèse d'optimisation locale n'est qu'asymptotiquement exacte et qu'elle donne d'assez bonnes prédictions pour les tailles expérimentalement pertinentes.

2.2. Fluctuations QUantiques à température Finie : FluCtuations QUANTIQUES TRONQUÉES. - Expliquons maintenant pourquoi et comment on peut réintroduire les fluctuations quantiques i.e. le fait que, de site en site de localisation, $\frac{1}{\xi}$ est distribué. Il faut pour cela se focaliser sur les prédictions relatives à un échantillon donné lorsque l'on varie soit la tension de grille soit la température.

2.2.1. Variations de température. - Le point majeur de l'analyse des sections précédentes est que la résistance globale d'un échantillon se trouve dominée par un seul saut entre sites localisés entre lesquels le transport est le plus difficile $[21,22,27]$. Qualitativement, on peut distinguer deux types de fortes résistances:

- ou bien la forte résistance surgit du fait de la grande distance $\simeq \eta_{\max } \xi$ entre les deux impuretés dont les énergies sont voisines (type I) ;

- ou bien la forte résistance surgit du fait de la grande différence d'énergie entre les niveaux, qui sont par ailleurs spatialement proches (type II).

Supposons qu'à une certaine température $T_{1}$, le lien le plus résistif soit de type II. Sa résistance augmente très vite à mesure que la température décroît. Il devient

$\left({ }^{4}\right)$ Pour effectuer cette comparaison entre la figure 4 et [21], du fait de différences de notation, on doit poser $T_{0}^{*}=0,04$ et $r_{0} \equiv \frac{\eta_{0} \xi}{4}$. 


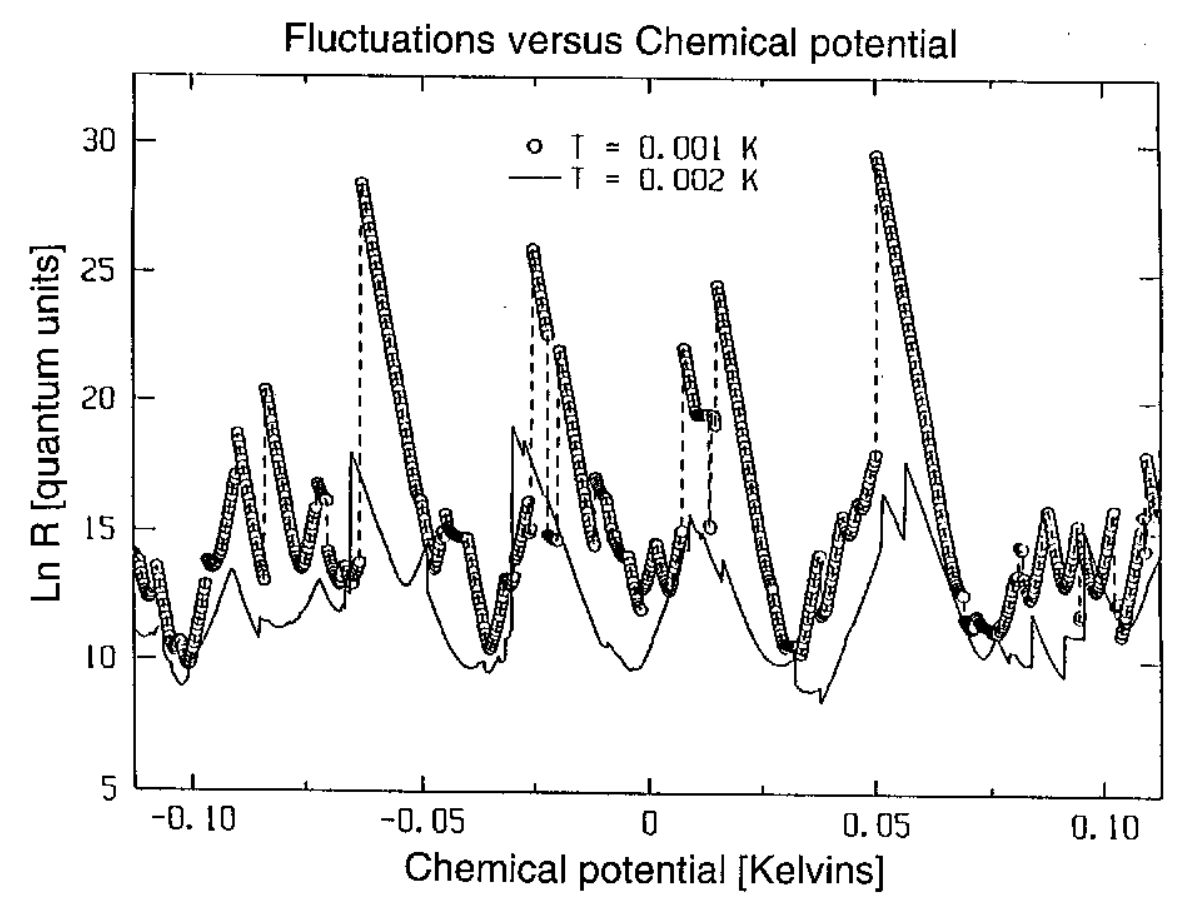

Fig. 4. - Simulation des fluctuations géométriques en fonction de l'énergie de Fermi, en utilisant les mêmes paramètres que Lee : $\frac{L}{\xi}=1000, \xi=50 l_{\text {scat. }}$, densité d'états $=1$ par Kelvin. La comparaison avec les résultats de Lee révèle des différences entre la forme des fluctuations obtenues par percolation et celle obtenue par la méthode d'optimisation locale. Soulignons que les pentes verticales - responsables du croisement des courbes à différentes températures — sont des artefacts de la procédure d'optimisation locale. D'après [28].

[Simulation of geometrical fluctuations versus Fermi energy, using the same parameters as in Lee's simulations: $\frac{L}{\xi}=1000, \xi=50 l_{\text {scat. }}$, density of states 1 per Kelvin. Comparison with Lee's work reveals differences between the shape of fluctuations obtained by the percolation method and by local optimisation. Note that the vertical slopes - which are responsible for the crossings of curves at different temperatures - are artefacts of the local optimisation procedure. From [28].]

donc de plus en plus probable que ce lien soit court-circuité par un autre lien dont la résistance est plus faible. En d'autres termes, en baissant la température on finira par changer de chemin de conduction, ou en tout cas de résistance dominante. Pour simplifier, supposons que c'est un lien de type I qui remplace un lien de type II lorsque la température décroît. En appelant $T_{2}$ la température à laquelle se fait ce "changement de dominance", on peut montrer, en utilisant des arguments 
identiques à ceux de la figure 3 - où l'on a calculé la probabilité qu'une aire donnée du plan $x, E$ soit vide de niveaux localisés -, que:

$$
\frac{\left(T_{1}-T_{2}\right)}{T_{2}} \simeq \frac{1}{\ln (a N)}
$$

Réciproquement, un lien de type I ("long et de faible énergie") perdra son statut de lien le plus résistif à mesure qu'augmente la résistance du second lien le plus résistif (le lien "sous-dominant") que pour simplifier nous supposerons de type II. L'intervalle de température à la fin duquel se produit ce changement est aussi donné par la formule précédente.

Il apparaît donc que le comportement en température d'un échantillon donné devrait être du type de ce qui est schématisé sur la figure 5. À basse température, on prévoit une succession du type "région activée-région en plateau", oscillant autour d'une loi de Mott dont la pente varie avec la taille $\left(\eta_{0} \sqrt{2 \ln (a N)}\right)$. La taille de ces régions est approximativement constante en échelle logarithmique et tend vers zéro comme $\frac{1}{\ln (a N)}$ lorsque $N$ tend vers l'infini.

2.2.2. Variations de niveau de Fermi (i.e. de tension de grille). - Reprenant des arguments déjà évoqués par Lee [21] ainsi que par Raikh et Ruzin [27], on constate d'après la relation exprimant la résistance associée à un saut entre des sites $\left(x_{i}, E_{i}\right)$ et $\left(x_{j}, E_{j}\right)$, que la variation de $E_{\mathrm{F}}$ demande de distinguer deux cas :

- soit $E_{i}$ et $E_{j}$ sont du même côté de $E_{\mathrm{F}}$ et alors $\ln (R)$ varie comme $\pm \frac{E_{\mathrm{F}}}{T}$ lorsque l'on varie $E_{\mathrm{F}}$;

- soit $E_{i}$ et $E_{j}$ sont de part et d'autre de $E_{\mathrm{F}}$ et alors $\ln (R)$ est constant lorsque l'on varie $E_{\mathrm{F}}$.

La question est de traduire ceci afin de trouver la taille typique de variation de l'énergie de Fermi $\Delta E_{\mathrm{F}}$ sur laquelle un lien reste dominant. Supposons que l'on soit dans les cas où le lien dominant varie lorsque $E_{\mathrm{F}}$ varie. Lorsque cette variation de la résistance dominante sera de l'ordre de la largeur de la distribution de $n_{\max }$ alors on aura "changement de dominance" à la manière de ce que nous avons dit pour l'étude en température. Puisque la largeur $w\left(\eta_{\max }\right)$ de la distribution du logarithme de la résistance dominante est d'ordre $w\left(\eta_{\max }\right) \simeq \frac{\eta_{0}^{2}}{\eta_{\max }}$, on obtient :

$$
\Delta E_{\mathrm{F}} \simeq \frac{\frac{\eta_{0}^{2}}{\eta_{\mathrm{max}}}}{\frac{1}{2 T}} \simeq \frac{2 \eta_{0} T}{\sqrt{2 \ln (a N)}}
$$

En fait, on s'aperçoit que cette valeur est typiquement l'écart moyen entre niveaux d'énergie à l'échelle $r_{\max }$. Mais des simulations récentes ont montré [31], que à $T=0 \mathrm{~K}$ l'amplitude typique en énergie des fluctuations quantiques de la conductance de systèmes localisés est précisément donnée par l'écart entre niveaux 


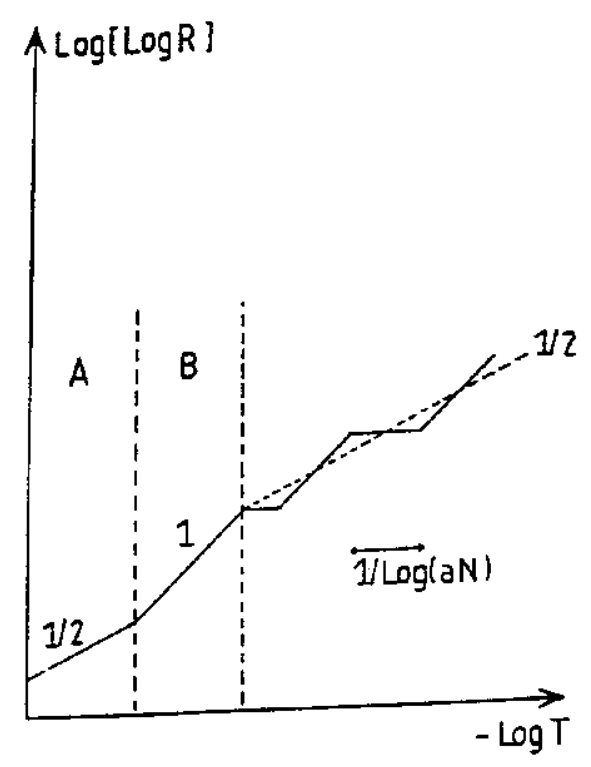

Fig. 5. - Évolution schématique de $\ln (\ln R)$ suivant $-\ln T$. Pour les échantillons suffisamment longs, on devrait avoir trois régions : A : loi de Mott $1 d$ valide avec donc une pente $\frac{1}{2}$ sur le graphe ; $\mathrm{B}$ : région où $R$ est auto-moyennante, mais où $\eta_{0}$ est suffisamment grand pour avoir une pente 1 ; $\mathrm{C}$ : région où $R$ est dominée par le saut mésoscopique le plus résistant, constituée d'une succession de plateaux (de pente nulle) et de régions activées (de pente 1), autour d'une loi de Mott effective (en pointillé) que l'on ne retrouve qu'après moyenne d'ensemble sur un grand nombre d'échantillons microscopiquement différents. D'après [28].

[Evolution of $\ln (\ln R)$ with $-\ln T$ (schematic). For sufficiently long samples, one should see three regions: A: Mott, with slope $\frac{1}{2}$, B: Self-averaging regime, but with large enough values of $\eta_{0}$ to get a slope 1 on the graph, C: weak link dominated region, with a succession of plateaus (slope 0) and activated regions (slope 1), around an effective Mott law (dotted line), the latter being recovered when averaging over a large ensemble of mesoscopic samples. From [28].]

du système entier (supposé quantiquement cohérent). Qualitativement ceci peut s'expliquer en disant que la transmission change brutalement autour de chaque résonance. Faisant l'hypothèse usuelle que la cohérence quantique est préservée à l'échelle du saut de Mott, on peut conclure que les fluctuations quantiques à l'intérieur du saut dominant doivent être prises en compte car leur largeur typique en variation de $E_{\mathrm{F}}$ est de l'ordre de celle des fluctuations classiques, purement induites par les effets géométriques. En effet, puisque la résistance d'un échantillon donné est celle de son lien le plus résistif, les fluctuations quantiques ne pourraient être négligées que si elles variaient bien plus lentement que les fluctuations "géométriques", 
ce qui en moyenne n'est pas vrai. Bien sûr, de tels arguments ne traitent que de grandeurs moyennes. C'est pourquoi, l'origine précise (géométrique ou quantique) d'une fluctuation donnée demandera à être examinée dans chaque cas. $\mathrm{Si}$, expérimentalement, l'amplitude de la fluctuation en question est fortement dépendante de la température, alors on dira que cette fluctuation est d'origine géométrique car dans la relation donnant la résistance associée au saut entre deux états la variation de $T$ produit forcément un fort changement de la résistance. Au contraire, une fluctuation d'origine quantique sera indépendante de la température sur une large gamme, essentiellement parce qu'elle est due à une fluctuation du terme $\propto \xi^{-1}$ avec $E_{\mathrm{F}}$, au lieu d'être due à une variation des termes d'activation avec $E_{\mathrm{F}}$. La gamme de température sur laquelle une telle fluctuation quantique reste indépendante de la température est sensiblement celle sur laquelle un lien reste dominant (voir plus haut au paragr. 2.2.1). Autrement dit, les fluctuations d'origine quantique ne se résorbent que lorsque la température est suffisamment forte pour que le chemin de conduction change et/ou que la résistance de l'échantillon soit de moins en moins dominée par la résistance mésoscopique la plus forte.

Considérons maintenant une fluctuation de conductance qui soit purement d'origine quantique. En fait, une telle fluctuation ne pourra pas se développer complètement, pour des raisons similaires à celles mentionnées ci-dessus. Imaginons que, en variant $E_{\mathrm{F}}$, on rencontre une résonance diminuant beaucoup la résistance du lien dominant $R_{\text {dom. }}$. Alors, il arrivera que ce lien ne restera plus le plus résistif et sera remplacé par le second lien le plus résistif $\left({ }^{5}\right)$ (i.e. le lien sous dominant $R_{\text {sous-dom. }}$. deviendra dominant). Réciproquement, si les interférences quantiques renforcent la résistance dominante, un lien finira par court-circuiter la résistance devenue trop forte, limitant aussi l'augmentation de la résistance. Pour estimer l'amplitude de chacun de ces deux mécanismes de "troncature", il suffit de rappeler que:

$$
\left\langle\ln \left(R_{\text {dom. }}\right)-\ln \left(R_{\text {sous }- \text { dom }}\right)\right\rangle=w\left(\eta_{\max }\right) \simeq \frac{\eta_{0}^{2}}{\eta_{\max }} \simeq \frac{\eta_{0}}{\sqrt{2 \ln (a N)}}
$$

où l'on rappelle que $\eta_{0}=\left(\frac{T_{0}^{*}}{T}\right)^{1 / 2}, N$ est le nombre de sauts de Mott, et $a \simeq 2$ est une constante. On conclut donc que l'on observe directement (du fait de la dominance d'un seul lien) les fluctuations quantiques que lorsqu'elles sont inférieures à $\simeq \frac{\eta_{0}^{2}}{\eta_{\max }}$. Lorsque ces fluctuations sont plus fortes, elles sont tronquées de manière auto-cohérente par la présence d'autres liens. En particulier, on peut penser que, sauf pour des échantillons très courts aux plus basses températures, on n'observe jamais de vraies résonances quantiques, contrairement à ce qui avait été soutenu dans la référence [25].

$\left(^{5}\right)$ Nous négligeons la probabilité que la résonance en énergie de $R_{\text {dom }}$ se produise simultanément avec celle de $R_{\text {sous-dom. }}$. 
Nous avons donc atteint la conclusion que on observe des "fluctuations quantiques tronquées" lorsque :

$$
w_{\text {geo. }} \gg w_{q}>\frac{\eta_{0}^{2}}{\eta_{\max }}
$$

Il reste à traduire la condition ci-dessus en termes de valeurs de $\eta_{0}$. Nous avons déjà vu, à la fin du paragraphe 2.1.2.1, que la première inégalité revenait à supposer que l'on est pas trop proche de la transition, i.e. que l'on n'a pas $\eta_{0} \simeq 1$. De plus, $[5,7]$, on peut estimer que :

$$
w_{q} \simeq C_{\omega}\left(\frac{r}{\xi}\right)^{\omega} \simeq \eta_{\max }^{\omega}
$$

où $\omega=\frac{1}{2}$ selon l'approche des matrices aléatoires [7], alors que $\omega \simeq \frac{1}{5}$ selon l'approche des chemins dirigés en dimension $d=3[5]$. Traduisant en termes de taille la seconde inégalité, on trouve donc (en prenant par simplicité $C_{\omega}=1$ ) que les fluctuations quantiques sont tronquées dans les systèmes où le nombre de sauts $N$ est suffisamment grand, i.e. lorsque :

$$
\ln (a N)>\eta_{0}^{\frac{1-\omega}{1+\omega}}
$$

Affinant le raisonnement, on pourrait se demander si les prédictions de matrices aléatoires [7] concernant l'amplitude des fluctuations sont directement applicables au lien dominant. En effet, ce lien est caractérisé par l'absence de niveaux d'énergie autour de l'énergie de l'électron, alors que les approches de matrices aléatoires ne comportent aucune restriction sur la position des niveaux d'énergie. Une étude numérique de ce point serait intéressante, mais elle n'existe pas à notre connaissance. Peut être la mise en place d'une contrainte supplémentaire sur la position des niveaux d'énergie diminuerait sensiblement l'amplitude des fluctuations. Cependant, les fluctuations quantiques ne peuvent pas être réduites au point de devenir plus faibles que celles prédites par l'approche des chemins dirigés [5]. En effet, toute la justification de cette approche est justement de supposer l'absence de niveaux localisés au voisinage du niveau de Fermi (qui est l'énergie du site initial et final du saut). Or un des résultats de cette approche est de trouver que l'amplitude des fluctuations est indépendante de l'écart $W$ en énergie entre le niveau de Fermi et les sites intermédiaires. Bien qu'intéressant en soi, un calcul précis de l'amplitude des fluctuations quantiques sur le lien dominant n'est pas nécessaire ici. En effet, dans beaucoup de situations expérimentales on se trouve — sauf aux plus basses températures - dans la situation de fluctuations quantiques tronquées que l'on prenne $\omega=\frac{1}{2}$ ou que l'on prenne $\omega=\frac{1}{5}$.

2.2.3. Travaux expérimentaux et questions ouvertes. - La plupart des travaux expérimentaux que nous connaissons sont cités en référence [23,24]. Le point commun à toutes les études est que les fluctuations reproductibles de conductance dans les isolants quasi $1 \mathrm{~d}$ sont géantes - plusieurs ordres de grandeur - et qu'elles se résorbent à "haute température" - T de 1 à quelques Kelvins - 
Cependant, il est impossible de comparer très précisément les données expérimentales des différents travaux. En effet, le cadre théorique de la conduction dans les isolants quasi $1 \mathrm{~d}$ a évolué avec un certain retard par rapport aux mesures expérimentales. De ce fait, les grandeurs extraites des mesures testent le plus souvent des modèles théoriques anciens et ne permettent pas de questionner les modèles les plus récents. Pour illustrer notre propos, on peut prendre deux exemples. En premier lieu, l'effet du champ magnétique sur les fluctuations de conductance. Les premiers travaux de Fowler et al. [23] ont interprété les fluctuations de conductance induites par le champ magnétique comme un effet Zeeman décalant les niveaux d'énergie. Il a fallu attendre l'apparition du modèle des chemins dirigés et les expériences d'Orlov et al. [32] pour que ce modèle d'effets de spin soit abandonné au profit d'effets d'interférences le long du saut de Mott, interférences fortement sensibles au champ magnétique qui modifie la phase des trajectoires de diffusion.

En second lieu, citons la polémique théorique entre les modèles d'effet tunnel résonnant à travers tout l'échantillon — effets purement quantiques —, et les modèles de fluctuations de chemin dominant - purement classiques - que nous avons exposé au paragraphe 2.1. Un certain nombre de travaux ont été nécessaires avant d'arbitrer - grâce aux mesures de Webb et al. en 1985 - en faveur des modèles classiques. Le concept de fluctuations quantiques tronquées, introduit par Ladieu et Bouchaud renouvelle le débat en suggérant qu'il est nécessaire de réintroduire les fluctuations quantiques uniquement sur le lien dominant la conduction, ce qui constitue un dépassement de la polémique antérieure et demande pour être testé que l'amplitude de chaque fluctuation induite par la tension de grille soit enregistrée très précisément en fonction de la température. Ce concept de fluctuations quantiques tronquées n'a été pour l'instant testé que sur un seul système : des fils de Ga:As dopés au silicium [24]. En dépit de sa pertinence vis-à-vis de ces expériences, il serait souhaitable que ce concept soit testé sur d'autres systèmes.

2.3. CAS DES SystÈmes COURTS ET (TRÈs) LARgES. - Dans le cas quasi 1d, que nous venons d'étudier, le chemin de conduction est transversalement confiné à une échelle très inférieure à $\xi_{\mathrm{p}}$ ce qui rend inévitable certains liens bien plus résistants que ne le prédit l'optimisation de Mott. Que se passe-t-il lorsque l'échantillon est de grande section transverse mais que sa longueur $L_{x}$ - dans le sens du courant - est inférieure à $\xi_{\mathrm{p}}$ ? A priori on s'attend à ce que le transport soit dominé par des chemins moins résistants que ne le prédit la loi de Mott, puisque $\xi_{\mathrm{p}}$ est l'échelle à laquelle on rencontre forcément au moins une résistance se comportant selon l'optimisation de Mott. Quelles sont les caractéristiques de ces chemins "peu résistants" ? Combien y en a t il pour une section transverse $S_{\perp}$ donnée ? Nous abordons ces questions en exposant les grandes lignes de travaux de Raikh et Ruzin qui furent les premiers à cerner le problème avec une élégance et une concision qu'il convient de souligner [19,27].

2.3.1. Films de très grande section transverse. - Considérons la figure 6 sur laquelle un film court et de grande section transverse est schématisé. Puisque le fil est court, il doit exister des chemins qui minimisent la résistance mieux que ne 


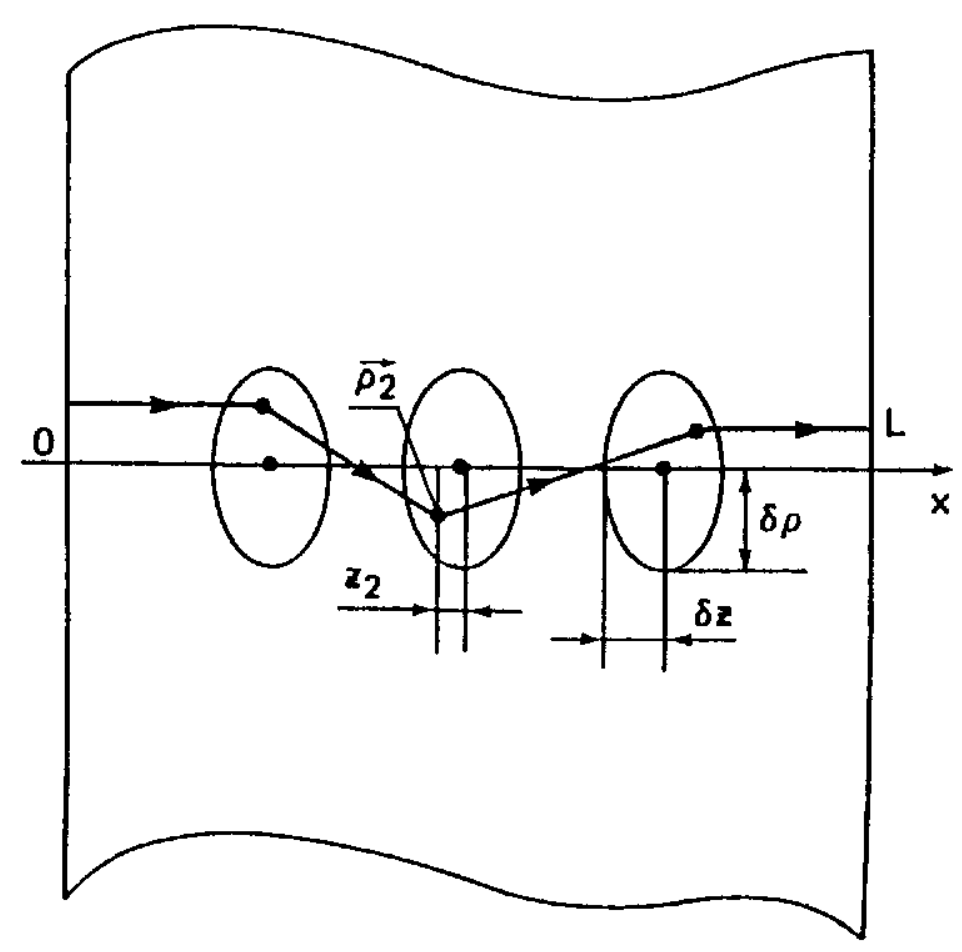

Fig. 6. - Film "court et de grande section transverse". La chaîne rectiligne représente le chemin idéal le moins résistant possible parmi tous les chemins à quatre sauts traversant l'échantillon. La chaîne non rectiligne est une chaîne optimale s'écartant de moins de $\delta \rho, \delta x, \delta E$ de la chaîne idéale. D'après [27].

[Short film of large transverse section. The straight chain is the ideal path, i.e. the least resistive among all the paths of four steps crossing the sample. The other chain is an optimal one differing from the straight one of less than $\delta \rho, \delta x, \delta E$. From [27].]

le propose l'optimisation de Mott, dont on n'a vu qu'elle ne devient meilleure solution qu'à l'échelle $\xi_{\mathrm{p}}$. Comment donc optimiser la résistance d'un tel système ? Une bonne approximation du problème est de rechercher les chemins qui sont tous constitués de résistances identiques. En effet, on a vu dans la section précédente que dès que les paramètres de saut augmentent un peu, la résistance locale associée augmente beaucoup et se met à dominer la résistance globale. Bien sûr de tels chemins, qui soient à la fois uniquement constitués de résistances identiques et plus conducteurs que l'optimum de Mott, sont extrêmement rares, et même statistiquement inexistants dès que la longueur du système excède $\xi_{\mathrm{p}}$ d'après ce qu'on a dit au paragraphe 1 sur les systèmes macroscopiques. Cependant, l’idée est que dans les systèmes courts $\left(L_{x}<\xi_{\mathrm{p}}\right)$ et de grande section transverse ce genre de chemin existe et que c'est lui qui domine la conduction. A priori, la probabilité d'occurrence d'un 
chemin à $N$ résistances identiques décroît lorsque $N$ augmente, mais on sait aussi que plus $N$ sera grand et plus la résistance sera faible car chaque saut élémentaire sera plus court. Le nombre $N$ de résistances identiques est donc, au départ, un paramètre qu'il s'agit d'optimiser en le prenant suffisamment grand pour que le chemin soit plus conducteur que l'optimum de Mott, mais aussi suffisamment petit pour que il y ait au moins un chemin de ce type dans le système de section $S_{\perp}$ considérée.

Sur la figure 6 , on a représenté - chaîne horizontale — une chaîne de sauts entre sites équidistants, séparés par la distance $\frac{L_{x}}{N}$. Si l'on ajoute la condition que tous les sites soient juste à l'énergie de Fermi, il est clair que l'on aura une chaîne de résistances dont la résistance globale est la plus petite que l'on puisse imaginer parmi les chaînes à $N$ résistances qui traversent l'échantillon. Cependant la probabilité qu'une telle chaîne existe dans un échantillon est rigoureusement nulle, puisque tous les paramètres de sauts sont fixés avec une précision absolue. Il nous faut donc, pour avoir une fréquence d'occurrence $w_{N}$ finie, autoriser certains écarts à la chaîne idéale aussi bien vis-à-vis de la distance entre sites que relativement à la position en énergie par rapport à $E_{\mathrm{F}}$. C'est ce qui est représenté sur la figure 6 par la chaîne non rectiligne, dont tous les sites sont situés à moins de $(\delta x, \delta \rho, \delta E)$ de ceux de la chaîne idéale. La probabilité qu’une telle chaîne non rectiligne apparaisse dans un échantillon est :

$$
w_{N}=\left[n\left(E_{\mathrm{F}}\right) \delta E(\delta \rho)^{2} \delta x\right]^{N}
$$

La résistance d'une telle chaîne est dominée par la résistance la plus forte, i.e. par le lien qui s'écarte le plus du lien idéal. Donc :

$$
R_{N}=R^{*} \exp \left(\frac{L_{x}}{N \xi}+\frac{2 \delta x}{\xi}+\frac{2(\delta \rho)^{2} N}{L_{x} \xi}+\frac{\delta E}{T}\right),
$$

où $R^{*}$ est de l'ordre du quantum de résistance et sera pris égal à 1 dans la suite.

Il s'agit désormais de sommer toutes ces chaînes favorables en parallèle et donc de sommer $w_{N} R_{N}^{-1}$. Du fait de la variation rapide de cette quantité, l'intégrale est dominée par le maximum de l'intégrant que l'on obtient en dérivant successivement par rapport à $N, \delta E, \delta x, \delta \rho$ qui sont quatre variables indépendantes. On trouve que le maximum de $w_{N} R_{N}^{-1}$ est atteint lorsque :

où

$$
\delta E_{\mathrm{opt}}=N_{\mathrm{opt}} T, \delta x_{\mathrm{opt}}=N_{\mathrm{opt}} \frac{\xi}{2}, \delta \rho_{\mathrm{opt}}=\sqrt{L_{x} \frac{\xi}{2}}
$$

$$
N_{\mathrm{opt}}=\sqrt{\frac{4 L_{x}}{\xi \lambda}} \text { avec pour définir } \lambda: \lambda=\ln \left(\frac{2 \lambda}{n\left(E_{\mathrm{F}}\right) T \xi L_{x}^{2}}\right)
$$

On reporte alors les paramètres optimaux que l'on vient de trouver dans la relation donnant la résistance de chaîne $R_{N}$ et l'on obtient une conductivité moyenne $\langle\sigma\rangle$ donnée par :

$$
\ln \langle\sigma\rangle=2 \sqrt{\frac{L_{x} \lambda}{\xi}}
$$


Autrement dit, on obtient une conductivité très peu dépendante de la température puisque dans l'expression ci-dessus seul $\lambda$ - paramètre sans dimension augmentant à basse température - dépend de $T$, et sa dépendance est très lente puisque en première approximation $\lambda \propto-\ln (T)$. Il convient de voir tout de suite les deux conditions de validité de cette expression de $\ln \langle\sigma\rangle$ :

- conditions sur $L_{x}$ : en premier lieu, cette valeur de conductivité ne peut pas convenir pour les échantillons trop courts, i.e. ceux dont la transmission tunnel $\frac{L_{x}}{\xi}$ directement de bord à bord est meilleure que celle proposée ici. Ceci impose $L_{x}>$ $\xi \lambda$. De plus, le calcul sur les chaînes optimales n'a de sens que s’il représente une conductivité plus forte que celle proposée par la loi de Mott $\ln \left\langle\sigma_{\text {Mott }}\right\rangle=\left(\frac{T_{0}}{T}\right)^{\frac{1}{d+1}}$. Cette condition impose que l'échantillon ne soit pas trop long, précisément que :

$$
L_{x}<L_{\mathrm{sup}}=\xi\left(\frac{T_{0}(d)}{T}\right)^{\frac{2}{d+1}}
$$

Soulignons que l'expression de $L_{\text {sup }}$ est différente de celle de la longueur de blob $\xi_{\mathrm{p}}$ introduite au paragraphe 1.3 en étudiant le travail de Pichard et Feng. En effet, on obtient :

$$
\xi_{\mathrm{p}}(d=3)=\xi\left(\frac{T_{0}(d=3)}{T}\right)^{0,36} \text { et } \xi_{\mathrm{p}}(d=2)=\xi\left(\frac{T_{0}(d=2)}{T}\right)^{\frac{5}{9}}
$$

Autrement dit $L_{\text {sup }}$ diverge plus vite que $\xi_{\mathrm{p}}$ à basse température. Or $L_{\text {sup }}$ est, dans cette analyse, l'échelle au delà de laquelle l'optimum de Mott devient meilleur que la conduction par chaînes de résistances toutes égales. On souhaiterait donc a priori que $L_{\text {sup }}$ soit comparable à $\xi_{\mathrm{p}}$, puisque cette dernière est l'échelle minimale pour que l'analyse d'Ambegaokar et al. soit valide ( $c f$. paragr. 1.3). Le fait que les exposants de $L_{\text {sup }}$ et de $\xi_{\text {p }}$ soient différents pose donc un problème $\left({ }^{6}\right)$ de raccordement entre l'analyse faite pour les systèmes macroscopiques et celle que nous venons d'exposer pour les systèmes courts et larges. On peut penser que cette différence est liée au fait que les deux méthodes utilisent des façons très différentes de construire le chemin optimal de conduction.

Par delà ce problème quantitatif sur la divergence de $L_{\text {sup }}$, l'analyse faite dans cette section nous permet de donner corps à ce que nous avions dit en introduction : lorsque l'échantillon est plus long qu'une taille critique, la meilleure optimisation est celle de Mott. Dans le cas contraire, les échantillons de grande section transverse ont une conductance supérieure à celle de Mott $\ln \langle\sigma\rangle=2 \sqrt{\frac{L \lambda}{\xi}}$ et peu dépendante de la température. Notons que, du fait de la divergence de la taille critique lorsque $T \rightarrow 0$, un échantillon de taille $L_{x}$ donnée finira toujours par présenter une conduction par chaînes optimales lorsque la température est suffisamment basse.

$\left({ }^{6}\right) \grave{A}$ notre connaissance ce problème n'est pas abordé dans la littérature. 
- condition sur la section transverse $S_{\perp}$ : lors du calcul sur les chaînes optimales, nous avons sommé en parallèle toutes les chaînes possibles, sans nous soucier que ces chaînes existent véritablement dans l'échantillon considéré. Ceci est valable si la section transverse de l'échantillon est très grande, au point que les divers types de chaînes (et surtout la chaîne optimale) soient représentées dans le système. Notant par $S_{0} \sim(\delta \rho)^{2} \sim\left(\frac{L_{x}}{N_{\text {opt }}}\right)^{2}$ la surface d'une chaîne optimale typique comportant $N_{\text {opt }}$ liens, le calcul fait ci-dessus pour $\ln \langle\sigma\rangle$ n'est valable que si :

$$
\frac{S_{\perp}}{S_{0}} w_{N_{\mathrm{opt}}, \delta E_{\mathrm{opt}} \gg 1}
$$

Reprenant l'expression de $w_{N}, N_{\text {opt }}, \delta E_{\text {opt }}$, on obtient la taille critique $S_{\mathrm{C}}$ au-dessus de laquelle le système sera de grande section (automoyennant) et décrit par le calcul de chaînes optimales ci-dessus. Il vient :

$$
S_{c}=\lambda L_{x} \xi e^{+\sqrt{\frac{L \lambda}{\xi}}}
$$

La section $S_{c}$ joue le même rôle que la longueur $N^{*}$ dans le cas des fils quasi $1 \mathrm{~d}$. C'est la taille au delà de laquelle le système devient automoyennant. Notons que $S_{c}$ diverge à basse température du fait de la divergence de $\lambda$. Tout échantillon de section transverse donnée finit donc par vérifier $S_{\perp}<S_{c}$. Il convient donc de voir ce qu'il se passe dans ce cas où le système n'est plus automoyennant.

2.3.2. Films de section transverse finie $\left(S_{\perp}<S_{c}\right)$. - Afin d'exprimer commodément que le film a une section transverse inférieure à $S_{c}$, Raikh et Ruzin introduisent le paramètre $\nu$ défini par :

$$
\nu=\frac{\ln \frac{S_{\perp}}{S_{0}}}{\ln \frac{S_{c}}{S_{0}}}
$$

Le cas des fils de section transverse finie revient donc à $\nu<1$. Dans ce cas là, la chaîne optimale — dont on a calculé les caractéristiques au paragraphe 2.2.1 — n'existe pas dans un échantillon typique. La conductance va être alors dominée par une seule chaîne, moins conductrice que celle du paragraphe 2.2.1, mais optimale pour l'échantillon considéré. Il n'est pas surprenant alors que la conductance fluctue fortement d'un échantillon à l'autre, reflétant la diversité des chaînes optimales entre deux échantillons. C'est pourquoi il convient, comme au paragraphe 2.1.2.2, de s'intéresser à ln $G$ car c'est une variable distribuée de façon étroite et la confrontation théorie/expérience est donc possible en pratique. Par ailleurs, nous nous intéressons à $\ln G$ et non plus à ln $\sigma$ pour la raison évidente que la conductivité n'a plus réellement de sens physique puisque la conduction est dominée par une seule chaîne de section transverse mésoscopique. Pour obtenir les paramètres typiques de la chaîne optimale, il convient d'écrire :

$$
\frac{S_{\perp}}{S_{0}} w_{N_{\nu}, \delta E_{\nu}}=1
$$


où $N_{\nu}$ représente le nombre de liens de la chaîne la plus conductrice, étant donné la contrainte $\nu<1$. Raikh et Ruzin obtiennent que les paramètres optimaux valent désormais :

$$
N_{\nu}=N_{\mathrm{opt}} \nu, E_{\nu}=\frac{E_{\mathrm{opt}}}{\nu}, \delta x_{\nu}=\frac{\delta x_{\mathrm{opt}}}{\nu}, \delta \rho_{\nu}=\frac{\delta \rho_{\mathrm{opt}}}{\nu}
$$

où les grandeurs indicées par "opt" ont été calculées au paragraphe 2.2.1. On déduit que la valeur typique du logarithme de la conductance vaut:

$$
\langle\ln G\rangle=\frac{\ln \left\langle G_{\mathrm{opt}}\right\rangle}{2}\left(\nu+\frac{1}{\nu}\right)
$$

Notons que cette expression tend vers ce que l'on a calculé au paragraphe 2.2.1 lorsque $\nu \rightarrow 1$. Bien sûr, la valeur typique du logarithme de la conductance dépend désormais de la température et cela pour deux raisons. D'abord parce que l'énergie d'activation de la meilleure chaîne n'est plus aussi petit qu'au paragraphe 2.2.1 puisque $\delta E_{\nu}>\delta E_{\text {opt }}$. Ensuite parce que $\nu$ diminue lorsque la température tend vers 0 , principalement à cause de la variation de $S_{c}$ avec $T$.

Par ailleurs, pour un échantillon donné, à mesure que l'on varie la température, la nature de la chaîne optimale se modifie de même que dans le cas quasi $1 \mathrm{~d}$ ( $c f$. Fig. 5 et paragr. 2.2.1). En effet, dans un échantillon donné la chaîne optimale n'est jamais rigoureusement identique à celle que l'on vient de calculer : en particulier une chaîne optimale reste dominante sur un certain intervalle de température $\tau$ et ne change pas continûment pour suivre l'estimation de la valeur typique de $\ln G$ que nous venons de faire ci-dessus. Ceci entraîne des fluctuations autour du comportement prévu pour $\langle\ln G\rangle$. Ces fluctuations ont pour "période" l'intervalle de température $\tau$ sur lequel une chaîne reste optimale. Raikh et Ruzin obtiennent :

$$
\tau=T \sqrt{\frac{\xi}{2 L} \ln \frac{S}{S_{0}}}
$$

La figure 7 représente ces changements de dominance de chaînes optimales à mesure que l'on varie la température. Bien que ce ne soit pas figuré sur la schéma extrait du travail de Raikh et Ruzin, on peut imaginer trois domaines de températures tout à fait analogues à ceux de la figure 5. À "haute température", $L_{x}>L_{\text {sup }}$ et $S_{\perp}>S_{c}$ ce qui rend la loi de Mott pertinente pour tout échantillon. A température plus basse, $S>S_{c}$ mais $L_{x}<L_{\text {sup }}$, ce qui rend possible la conduction par des chaînes optimales et ralentit fortement la dépendance de $\ln G$ avec la température. Ceci correspond au domaine $\mathrm{B}$ de la figure 5 où la conduction est dominée par le saut le plus résistant possible existant dans un fil quasi 1d. Enfin aux plus basses températures, des fluctuations de $\ln G$ apparaissent autour d'une dépendance moyenne en température : la conduction est alors assurée par la meilleure chaîne de chaque échantillon, ce qui entraîne de fortes fluctuations d'échantillon à échantillon. Ceci correspond au domaine $\mathrm{C}$ de la figure 5 et aux "plateaux" prédits autour du comportement typique de $\langle\ln R\rangle$. Cette analogie se révèle aussi par la dépendance inhabituelle de $\langle\ln R\rangle$ en fonction de la taille : pour les quasi 1d $\ln R \propto(\ln N)^{1 / 2}$ et pour les systèmes de grande section transverse $\langle\ln G\rangle$ dépend, par le biais de $\nu$, logarithmiquement de la section transverse de l'échantillon. 


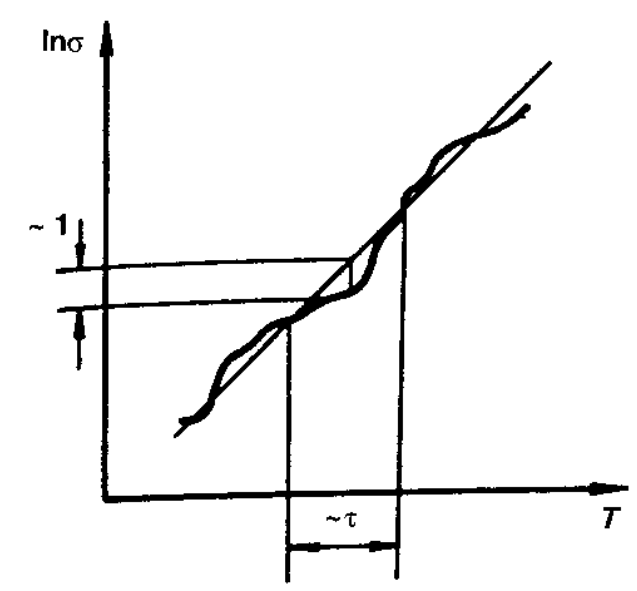

Fig. 7. - Fluctuations, autour d'une dépendance moyenne, de $\ln G$ pour un système court et large dans le régime des plus basses températures. D’après [19] et [27].

[Fluctuations, around a mean evolution, of $\ln G$ for a short and large system in the lowest temperatures regime. From [19] and [27].]

2.3.3. Confrontation aux travaux expérimentaux et questions ouvertes. - Sur le plan théorique, il n'existe pas d'approche percolative du problème du transport dans les systèmes de grande section transverse, contrairement au cas des quasi $1 \mathrm{~d}$. On ne peut donc pousser l'analyse aussi loin que nous l'avons fait aux paragraphes 2.1. Notons aussi, l'absence de la notion de "fluctuations quantiques tronquées" lorsque l'on varie la tension de grille $V_{\mathrm{G}}$, contrairement à ce que nous avons exposé dans le paragraphe 2.2. En effet, le modèle de Raikh et Ruzin est complètement classique au sens où il ne prend pas en compte le fait que $\xi$ peut varier avec la tension de grille, mais simplement que le chemin optimal peut changer. Comme nous l'avons dit plus haut - cf. paragraphe 2.2.3 - la notion de fluctuations quantiques tronquées ne peut être testée que par une étude extrêmement fine de l'effet de $T$ sur chaque fluctuation. Or, les travaux expérimentaux sur les systèmes courts et larges sont rares et ne s'attachent jamais à suivre l'évolution précise d'une fluctuation en température. Ces travaux ont été principalement menés sur des isolants bidimensionnels obtenus avec un gaz d'électrons $2 \mathrm{~d}$ où le bord de mobilité est franchi par l'application d'un potentiel de grille. Nous citerons deux séries de travaux : ceux d'Orlov et al. et ceux de Popovic et al.

Orlov et al. [32] se focalisent surtout l'effet de l'application d'un champ magnétique $H$ sur la conduction par sauts à portée variable. Les auteurs comparent les fluctuations induites par $H$ à celles induites par une variation de la tension de grille $V_{g}$. Il apparaît que les premières sont bien plus fortes que les dernières, ce qui amène les auteurs à supposer que l'effet de la grille est de changer le chemin dominant la conduction alors que celui du champ magnétique est seulement de modifier les 
interférences quantiques dans les sauts électroniques sans changer le chemin dominant. Une telle faiblesse des effets de champ magnétique avait été prévue dans le cadre du modèle dit des chemins dirigés par Nguyen et al. [4], contrairement aux modèles dérivés des matrices aléatoires qui prédisent une équivalence entre les deux types de fluctuations [7]. L'étude d'Orlov et al. [32] est donc venue confirmer la pertinence du modèle des chemins dirigés dans le régime très isolant. Depuis une autre étude - Ladieu et al. [24] — a confirmé ce résultat et montré que près de la transition les deux types de fluctuations redeviennent équivalentes. Enfin, signalons que l'effet moyen de $H$ est d'augmenter la conductance, ce que tous les modèles dans ce cas sans couplage spin orbite - interprètent comme une augmentation de la longueur de localisation avec $H$.

Orlov et al. [32] se préoccupent assez peu des effets de température, même si qualitativement, ils interprètent leurs observations dans le cadre du modèle de chaines optimales que nous venons d'exposer. Cependant, du fait que dans leurs expériences $T \geq 1,5 \mathrm{~K}$, il est impossible de tester précisément le modèle de Raikh et Ruzin. En particulier, les fluctuations de $\ln G$ autour d'une dépendance moyenne lorsque l'on varie la température ne sont pas reportées, pas plus que l'évolution précise en température de quelques fluctuations selon la tension de grille - ce qui aurait permis de tester la notion de fluctuations quantiques tronquées - .

Popovic et al. ont publié deux études expérimentales dans lesquelles ils étudient le transport dans deux systèmes isolants désordonnés courts et larges [33, 34]. La première est menée sur une couche d'inversion - dans un MOSFET en silicium - de section transverse et de longueur millimétriques [33]. Des fluctuations de conductance, induites par la tension de grille, sont mesurées à 4 températures différentes entre $90 \mathrm{mK}$ et $555 \mathrm{mK}$. Les auteurs montrent surtout l'histogramme des conductances - réalisé à partir des divers pics de conductance - et le confrontent aux prédictions de Raikh et Ruzin. L'accord est qualitativement satisfaisant. Notons que les auteurs soulignent que leur expérience est cantonnée au cas $\nu>1$, i.e. aux systèmes suffisamment larges pour être automoyennants. C'est pourquoi, il n'est pas étonnant qu'aucun comportement du type de celui de la figure 7 ne soit observé, ce qui est dommage car c'est une des prédictions les plus spectaculaires de Raikh et Ruzin. Le deuxième travail [34] de cette équipe étudie des systèmes encore plus petits : sur un gaz bidimensionnel désordonné ont été définies des échantillons de longueur micronique sur 10 à 100 microns de large. Les fluctuations de conductance y sont étudiées jusqu'à $10 \mathrm{mK}$. Les auteurs ne reportent pas non plus de comportement du type de la figure 7 car ils concentrent leur interprétation sur deux points majeurs. En premier lieu, aux plus basses températures, $N_{\text {opt }}$ est de l'ordre de 1 ce qui signifie que le transport s'effectue par effet tunnel résonnant à travers tout l'échantillon, ce que confirme la comparaison des mesures au modèle de Glazman et al. [35]. En second lieu, les auteurs enregistrent une augmentation de la conductance avec la longueur des échantillons, ce qui est, selon les auteurs eux mêmes, non seulement contre intuitif mais en plus quasi inexpliqué théoriquement à ce jour.

Pour résumer cette section 2, rappelons que lorsque le chemin de percolation ne peut pas se développer librement du fait de la taille du système, la loi de Mott 
n'est pas valable. Dans le cas quasi $1 \mathrm{~d}$, la meilleur chemin de conduction est dominé par sa résistance mésoscopique la plus forte, ce qui rend la résistance mesurée plus divergente que ne le prédit la loi de Mott lorsque $T \rightarrow 0$. Symétriquement, dans les systèmes courts et larges, il existe des chaînes qui court-circuitent le chemin d'Ambegaokar et al., ce qui rend la résistance moins divergente que la prédiction de Mott. Le point commun à ces deux situations est que la résistance mesurée est essentiellement donnée par un très petit nombre de sauts mésoscopiques, parfois un seul. Les caractéristiques typiques de ce saut dominant évoluent avec la taille du système ce qui produit une dépendance anormalement lente de la conductance avec la taille du système. Le fait que la conductance mesurée soit dominée par un seul lien rend les expériences extrêmement sensibles à tout ce qui va affecter ce

saut dominant: changement pur et simple de chemin par variation de l'énergie de Fermi $E_{\mathrm{F}}$ et/ou variation des interférences quantiques affectant la transmission sur le lien dominant. L'arbitrage entre ces deux effets a suscité de nombreux travaux et le modèle le plus récent à ce sujet est celui des fluctuations quantiques tronquées qui demande a être plus systématiquement testé qu'il ne l'a été jusqu'à présent.

\section{La si difficile question des interactions}

À part le principe d'exclusion de Pauli inclus dans le calcul des conductances locales d'Halperin et al. (cf. paragr. 1) nous n'avons en aucune autre façon tenu compte de l'interaction entre électrons dans les isolants désordonnés. En particulier nous avons jusqu'ici négligé l'interaction coulombienne répulsive entre deux électrons. Pour tenter d'aborder cette question, nous commençons par exposer, très succinctement, l'effet des interactions en l'absence de désordre car on sait, depuis les travaux de Mott [36], qu'à elles seules les interactions entre électrons peuvent induire une transition métal-isolant. Nous effleurons la physique du blocage de Coulomb qui nous permet d'insister sur la notion d'énergie de charge et d'en donner une approche intuitive aussi bien sur une seul grain métallique que sur un réseau de grains. Armé de ces notions, nous serons à même d'exposer et de commenter les divers modèles élaborés au sujet du comportement en régime linéaire d'une part et non linéaire d'autre part. Nous distinguons les modèles homogènes, i.e. n'utilisant qu'une longueur fondamentale - à savoir $\xi$ - des modèles granulaires qui utilisent au moins deux longueurs. Nous insistons sur la loi d'Efros et Shklovskii qui fut longtemps la seule prédiction au sujet de la résistance - en régime linéaire tenant compte à la fois des interactions et du désordre. Nous terminons en abordant un travail plus récent d'Ortuno et al. dont nous suggérons qu'il est peut être un début de généralisation de la loi d'Efros et Shklovskii.

\subsection{Interactions entre ÉleCtrons et isolants DE MotT}

3.1.1. La transition métal-isolant de Mott. - Mott a été le premier à comprendre que les interactions entre électrons peuvent induire un état isolant. La première modélisation proposée par Mott a été la suivante. Considérons une assemblée d'atomes d'hydrogène dont la séparation peut être contrôlée [36]. Lorsque cette séparation 
est grande, on a un état isolant puisque chaque électron reste sur son atome quasiisolé et ressent le potentiel coulombien $V(r)=\frac{e}{r}$ dû à la charge $+e$ du noyau. Ce potentiel donne lieu à un état lié dont l'extension spatiale typique est donnée par le rayon de Bohr $a_{\mathrm{b}}=\frac{\hbar}{m e^{2}}$.

Cependant, dans une assemblée d'atomes, la charge du noyau est écrantée, ce qui réduit le potentiel à une valeur effective donnée par le potentiel de Yukawa :

$$
V(r)=\frac{e}{r} \exp \left(\frac{-r}{\lambda_{\mathrm{T} . \mathrm{F}}}\right)
$$

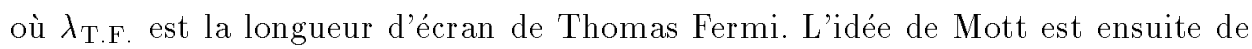
comparer les deux longueurs que sont $a_{\mathrm{b}}$ et $\lambda_{\mathrm{T}}$.F. . Si l'écrantage est faible, $\lambda_{\mathrm{T}}$.F. est grand et les électrons voient le potentiel nucléaire "nu", ce qui les rend fortement liés à leur atome d'origine. Le système est donc dans un état isolant. Inversement, lorsque le saut d'un site à l'autre est favorisé (i.e. lorsque la densité d'électrons est plus forte), c'est $\lambda_{\mathrm{T} . \mathrm{F}}$. qui est la longueur la plus petite, produisant un écrantage du potentiel nucléaire qui permet des états d'électrons "libres" ou en tout cas métalliques.

Le critère de transition est donc $\lambda_{\mathrm{T} . \mathrm{F}} \simeq a_{\mathrm{b}}$. En estimant $\lambda_{\mathrm{T} . \mathrm{F}}$. d'après le modèle du gaz d'électrons dans l'approximation de Thomas Fermi, on obtient comme critère de transition :

$$
n_{\mathrm{c}}^{1 / 3} a_{\mathrm{b}} \simeq 0,25
$$

Ce critère est très souvent vérifié dans l'expérience comme le montre la figure $8[37]$.

Plus techniquement, le raisonnement de Mott, qui fait jouer un rôle essentiel aux termes de Coulomb, peut se traduire par un hamiltonien de liaisons fortes:

$$
\mathcal{H}=\sum_{i, \sigma} \epsilon_{i} n_{i, \sigma}+\sum_{i, j, \sigma} V_{i, j} a_{i, \sigma}^{+} a_{j, \sigma}+U \sum_{i} n_{i, \sigma} n_{i,-\sigma}
$$

où $n_{i, \sigma}$ est l'opérateur nombre de particules au site $i$ dont le spin vaut $\sigma$. Notons que le désordre est absent du problème puisque aussi bien les énergies de sites $\epsilon_{i}$ que les intégrales de transfert $V_{i, j}$ sont uniformes, i.e. non distribuées. Les termes de Coulomb sont contenus dans le troisième terme du hamiltonien, le principe de Pauli étant satisfait du fait de l'opposition des spins de deux électrons situés sur le même site.

Schématiquement, la transition métal-isolant dans ce modèle peut être vue comme sur la figure 9. L'augmentation de la densité d'électrons revient à augmenter le terme de transfert $V$, à $U$ constant. Ceci produit un élargissement des bandes de Hubbard. Si l'on a un électron par site en moyenne, la bande inférieure est pleine et elle est séparée de la bande supérieure par un gap, ce qui rend le système isolant. Lorsque l'élargissement des sous bandes de Hubbard devient d'ordre $U$, les bandes se recouvrent et la densité d'états au niveau de Fermi n'est plus nulle. Le système est alors devenu métallique. 


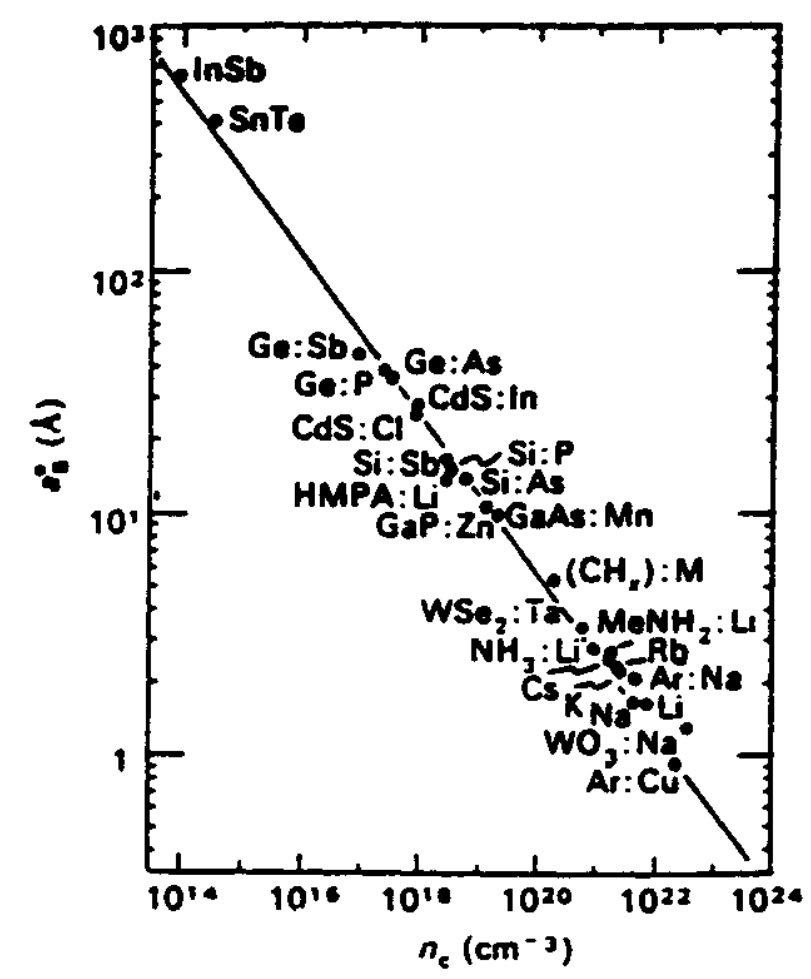

Fig. 8. - Divers systèmes satisfaisant le critère $n_{c}^{1 / 3} a_{\mathrm{b}} \simeq 0,25$ à la transition métalisolant $[37]$.

[Experimental data showing the relevance of Mott's criterium $n_{\mathrm{c}}^{1 / 3} a_{\mathrm{b}} \simeq 0,25$ at the MetalInsulator transition. From [37].]

Bien que Mott ait émis ces idées il y a plusieurs décennies, le problème de l'isolant de Mott reste à bien des égards un problème ouvert. Signalons les travaux de Georges et Kotliar [38] qui ont récemment proposé une approche analytique du problème de Mott-Hubbard en champ moyen qui devient exacte dans la limite $d \rightarrow \infty$ où $d$ est la dimensionalité du système. Cette approche a été ensuite étendue pour étudier l'effet d'un champ magnétique [39]. Avec la découverte des supraconducteurs à haute température critique, on a assisté à un nouvel engouement pour toutes ces questions car on pense que beaucoup de nouveaux supraconducteurs ont une phase isolante à proximité de leur phase supraconductrice. Par ailleurs, les nouvelles techniques de nano lithographie permettent de construire des structures artificielles qui sont suffisamment petites pour constituer des systèmes modèles d'isolant de Mott. C'est la physique du blocage de Coulomb, dont nous allons parler maintenant. 


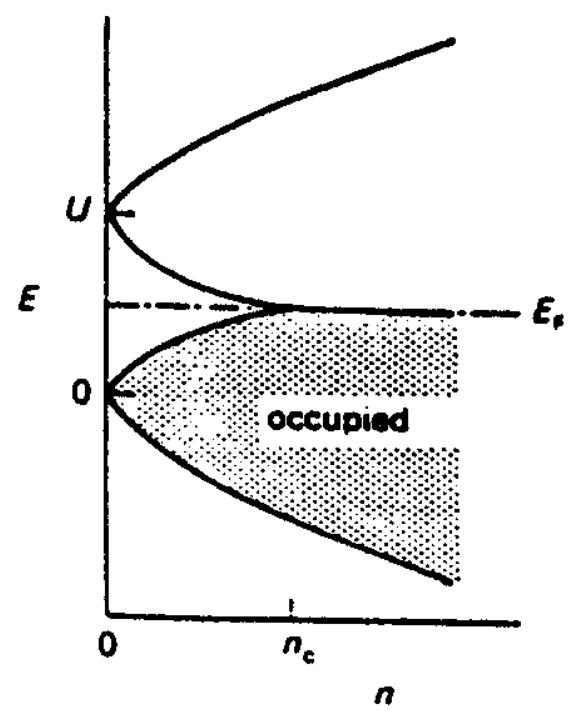

Fig. 9. - Transition métal-isolant selon le modèle de Hubbard. Le couplage aux premiers voisins augmente avec la concentration $n$, donnant lieu à des bandes qui se recouvrent à la transition.

[Metal-Insulator transition according to the Hubbard model. The nearest neighboor coupling constant increases with the concentration $n$, yielding two energy bands which overlap at the Metal-Insulator transition.]

3.1.2. Blocage de Coulomb sur une seule île. - Considérons, comme sur la figure 10, une île métallique séparée de deux réservoirs (i.e. deux fils de contacts) par deux barrières tunnels de transmission inférieure à 1, i.e. dont l'épaisseur est plus grande que la longueur d'amortissement tunnel. L'état fondamental de ce système est un état dans lequel le nombre de charges est un bon nombre quantique : même si les quasi-particules du système ne sont pas exactement des électrons "nus" (mais des électrons "habillés", entre autres, de phonons), les quasi-particules ont pour charge -e. L'idée principale est que toutes les excitations du système vont nécessiter de transférer au moins une charge e sur l'île, la faible transparence des barrières rendant en effet impossible de transférer moins qu'une charge entière [40,41]. Le prix énergétique de l'excitation de plus basse énergie va donc être d'ordre $\frac{e^{2}}{C}$, où $C$ est la capacitance totale entre l'île et les réservoirs. On aura donc un système ayant "un gap" entre son fondamental et ses excitations de basse énergie.

Pour que ce gap ne soit pas ridiculement faible en énergie il faut que $C$ soit suffisamment petit : pour avoir un gap de $1 \mathrm{~K}$, il faut $C \simeq 1 \mathrm{fF}$. Rappelons que l'on a $C \simeq 4 \pi \epsilon_{0} \epsilon_{\mathrm{r}} a$ où $a$ est le rayon de l'île et $\epsilon_{\mathrm{r}}$ est la constante diélectrique relative des barrières tunnels. Avec $\epsilon_{\mathrm{r}} \simeq 10$, on obtient $C \simeq 1 \mathrm{fF}$ lorsque $a \simeq 1 \mu \mathrm{m}$. Lorsque la température est inférieure au gap, on s'attend donc à ce que, soumis à un champ 


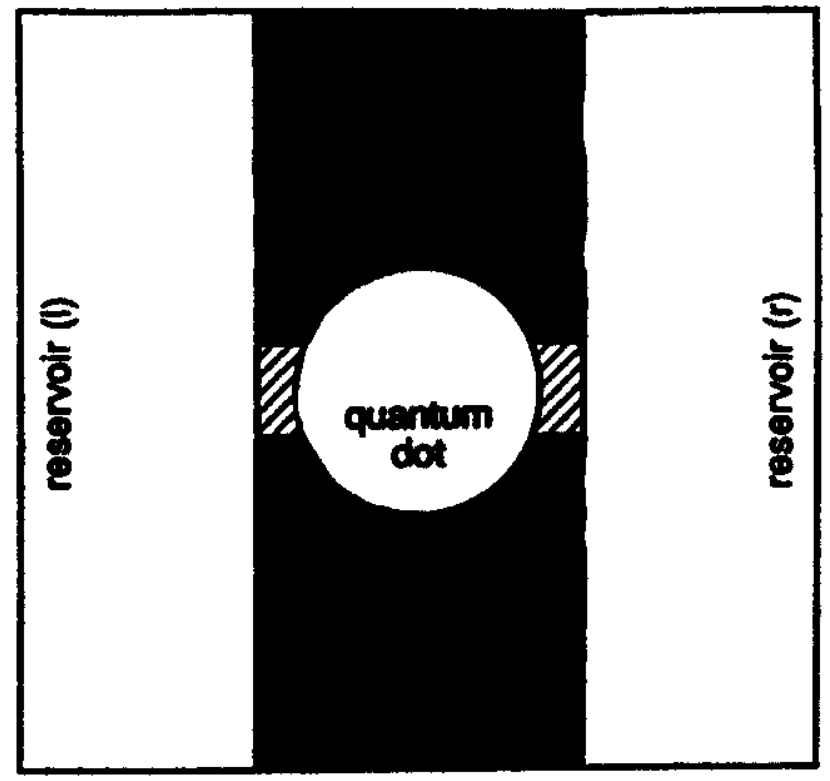

Fig. 10. - Représentation d'une île métallique (au centre) séparées de deux réservoirs par deux barrières tunnels (hachurées). D’après Beenaker C.W., Phys. Rev. B44 (1991) 1646 .

[Schematic view of a metallic island (quantum dot) linked to reservoirs by two tunnel barriers (hatched). From Beenaker C.W., Phys. Rev. B44 (1991) 1646.]

électrique, la résistance du système soit exponentiellement activée, i.e. on s'attend à avoir :

$$
R_{V \rightarrow 0} \propto \exp \left(\frac{\mathcal{U}}{T}\right) \text { où } \mathcal{U}=\frac{e^{2}}{C}
$$

où $V \rightarrow 0$ rappelle que la relation ci-dessus n'est valable qu'à très faible excitation.

Plus précisément, regardons la figure 11. Entre les deux réservoirs on a imposé une différence de potentiel $V$ par une source de tension. Au centre sont représentés les niveaux d'énergie à une particule, i.e. les niveaux d'énergie des quasi-électrons. Dans un premier temps nous négligerons l'écart $\Delta$ entre niveaux d'énergie à une particule ainsi que la différence de potentiel $V$ imposée par la source de tension. Pour évaluer le terme d'énergie électrostatique $U$ relatif à l'ensemble des $N$ électrons présents sur l'île, on cherche à évaluer au plus bas ordre le potentiel électrostatique $\phi(Q=N e)$ régnant sur l'île lorsque elle contient $N$ électrons. On utilise l'électrostatique la plus simple en considérant la capacitance totale $C$ entre l'île et les réservoirs et l'on obtient :

$$
\phi(Q)=\frac{Q}{C}+\phi_{\text {ext }}
$$




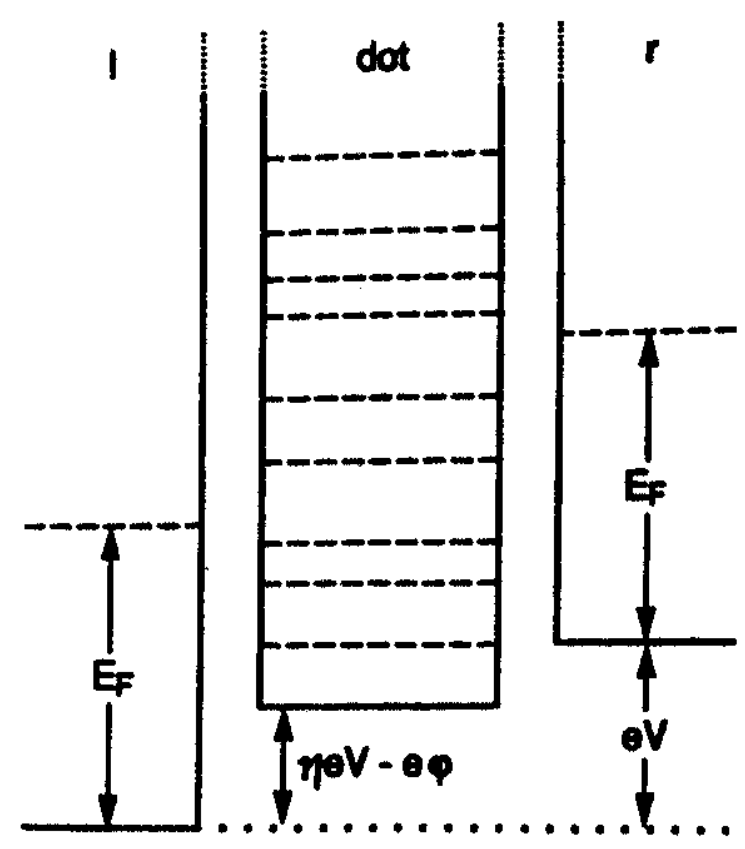

Fig. 11. - Schéma espace-énergie d'une île métallique dont les niveaux d'énergie (discrets) sont représentés par des pointillés. Cette île est séparée de deux réservoirs par deux barrières tunnels. Une tension $V$ est appliquée entre les deux réservoirs. $\phi$ est le potentiel résultant d'un possible couplage à une grille, $\eta$ est un nombre entre 0 et 1 traduisant l'asymétrie - entre les deux barrières - des coefficients de transmission quantique vers les réservoirs. D'après Beenaker C.W., Phys. Rev., B44 (1991) 1646.

[Space-energy diagram of a metallic island whose discrete energy levels are showed (dashed). This island is linked to reservoirs by two tunnel barriers. A tension $V$ is applied between the two reservoirs. $\phi$ is the potentiel resulting from possible capacitive coupling to a gate, $\eta$ is a number - between 0 and 1 - allowing to take into account the possible asymmetry of the coefficients of the quantum transmission of the two bariers. From Beenaker C.W., Phys. Rev. B44 (1991) 1646.]

où $\phi_{\text {ext }}$ est le potentiel électrostatique crée par ce qui est extérieur au gaz de $N$ électrons. Par exemple dans un gaz d'électrons bidimensionnel ce sera le potentiel dû aux atomes donneurs (situés le plus souvent hors de l'interface bidimensionnelle). $\phi_{\text {ext }}$ pourra contenir aussi le potentiel d'une grille déposée au dessus de l'île. Puisque l'on a :

$$
U(N) \equiv \int_{0}^{-N e} \phi\left(Q^{\prime}\right) \mathrm{d} Q^{\prime}
$$

on obtient :

$$
U(N)=\frac{N e^{2}}{2 C}-N e \phi_{\text {ext }}
$$


Introduisant $Q_{\text {ext }} \equiv C \phi_{\text {ext }}$, il vient :

$$
U(N)=\frac{\left(N e-Q_{\text {ext }}\right)^{2}}{2 C}-\frac{Q_{\text {ext }}^{2}}{2 C}
$$

Pour un potentiel $\phi_{\text {ext }}$ donné il n'y a donc qu'une seule valeur de $N$ qui minimise $U$. En gros, cette valeur correspond à la condition d'électroneutralité entre le fond de charges positives - contenues dans $\phi_{\text {ext }}$ - et les électrons contenus sur l'île. De plus, en formant la quantité $U(N)-U(N-1)$ on constate qu'elle est d'ordre $\frac{N e^{2}}{C}$. Cette énergie représente la somme de toutes les énergies d'interaction entre les $N$ électrons du gaz et la charge excédentaire lorsque la charge sur l'île a varié de un électron. Varier la charge d'une île de une unité coûte donc, par particule, une énergie $\frac{e^{2}}{C}$. On peut donc donner l'image suivante du transfert de charge dans ce genre de système :

- on prend une particule au niveau de Fermi ;

- on la transfère sur l'île. Ceci coûte une énergie $\frac{N e^{2}}{C}$, ce que l'on peut traduire en termes à une particule comme le fait que chacun des $N$ niveaux à une particule a vu son énergie augmenter de $\mathcal{U}=\frac{e^{2}}{C}$.

Ceci revient à dire que l'énergie à fournir pour transférer la particule sur l'île est $\frac{e^{2}}{C}$ (et cela même si l'île métallique contient des milliards d'électrons dans l'état fondamental). De là, on déduit la loi d'activation citée plus haut pour la résistance $R$.

Notons que, jusqu'ici, nous avons supposé la densité d'états à une particule suffisamment grande pour négliger l'écart $\Delta$ entre niveaux à une particule sur l'île. Ceci est souvent valable si l'île est constituée d'un métal normal et n'est pas de trop petite taille. En revanche, si l'île est constituée par un semiconducteur dopé, le plus souvent $\Delta$ ne sera pas négligeable devant $\frac{e^{2}}{C}$. Il faudra alors inclure $\Delta$ en tenant compte de chaque situation particulière : si le dernier niveau occupé sur l'île est situé à $E_{\mathrm{F}}-\Delta$, on aura à fournir $\frac{e^{2}}{C}-\Delta$ pour transférer une charge. Inversement, si le dernier niveau occupé sur l'île est situé à $E_{\mathrm{F}}+\Delta$, on aura à fournir $\frac{e^{2}}{C}+\Delta$ pour transférer une charge.

Il y aurait évidemment beaucoup à dire sur toutes les subtilités du blocage de Coulomb qui a été très étudié depuis que les progrès de la nano-lithographie ont permis de dessiner des îles suffisamment petites $(\sim 1 \mu \mathrm{m})$ pour que $\frac{e^{2}}{C}$ soit de l'ordre du Kelvin. Nous indiquons quelques références parmi les nombreuses possibles $[41,42]$. 
Retenons que le blocage de Coulomb sur une île est l'exemple le plus simple d'isolant de Mott puisqu'il ne comporte qu'un site. Le méthode très simple que nous avons employée pour tenir compte des interactions électron-électron sur l'île est bien entendu une approximation au plus bas ordre. Cependant, cette approximation permet de prédire que $R$ est simplement activée à suffisamment basse $T$, du fait de l'existence d'un "gap" dans les excitations du système. Ce "gap" est appelé "énergie de charge" et s'obtient tout simplement en calculant $\frac{e^{2}}{C}$.

3.1.3. Réseau de grains capacitivement couplés. - Bien que la physique du blocage de Coulomb sur une île soit intéressante en elle même, si l'on s'intéresse au problème de l'isolant de Mott, il faut considérer un modèle comportant un réseau de sites. Parmi les nombreux modèles existants, il faut distinguer ceux où le désordre est pris en compte de ceux qui ne contiennent absolument aucun terme de désordre.

3.1.3.1. Interactions sur réseau sans désordre. - Nous exposons ici les résultats d'un article récent [43], sans prétendre par ce seul exemple épuiser la richesse de la transition de Mott.

Les auteurs considèrent un réseau $d$-dimensionnel de grains capacitivement couplés $\left(d=1\right.$ ou $d=2$ ). Chaque grain comporte $M$ niveaux d'énergie $\epsilon_{\alpha}, \alpha=1$ à $M$, les valeurs des $\epsilon_{\alpha}$ étant les mêmes pour tous les grains (absence de désordre). L'indice $\alpha$ spécifie totalement l'état quantique, incluant les degrés de liberté de spin que l'on suppose non-dégénérés dans le cadre de ce modèle. Notant $n_{j}$ l'opérateur nombre de particules au site $j$ tous états $\alpha$ confondus, et $t_{\alpha}$ l'énergie gagnée par délocalisation lors du passage par effet tunnel d'un grain à l'autre, on écrit le hamiltonien suivant :

$$
\mathcal{H}=-\sum_{(i, j), \alpha}\left(t_{\alpha} c_{i, \alpha}^{+} c_{j, \alpha}+\text { c.c. }\right)+\sum_{j, \alpha} \epsilon_{\alpha} c_{j, \alpha}^{+} c_{j, \alpha}+\frac{U}{2} \sum_{j} n_{j}\left(n_{j}-1\right)
$$

où $U$ est le terme de "Hubbard par particule", i.e. l'analogue du terme d'ordre $\frac{e^{2}}{C}$ calculé plus haut. Par ailleurs l'écart énergétique $\Delta$ entre niveaux est pris constant, i.e. $\Delta=\epsilon_{\alpha+1}-\epsilon_{\alpha}, \forall \alpha$. De même, $t_{\alpha}=t, \forall \alpha$.

À l'aide de simulations numériques, les auteurs établissent le spectre énergétique des excitations de ce système à partir de l'état fondamental. Ils trouvent trois phases distinctes suivant les valeurs de $\frac{t}{U}$ :

- lorsque $t$ est petit, on a une phase de blocage de Coulomb individuel, i.e. l'addition d'un électron ne peut se faire que moyennant un apport d'énergie $U+\Delta$ car l'état fondamental est un état où le nombre de particules sur chaque ile est un bon nombre quantique. En d'autres termes, $t$ est trop faible pour que les fluctuations de charge par île soient d'ordre $e$;

- lorsque $t$ augmente, précisément lorsque $t>\Delta$, l'état fondamental n'est plus l'état où chaque électron est mis sur le niveau d'énergie le plus faible. Il devient énergétiquement favorable d'inclure, dans l'état fondamental du système, les états — à une particule — d'énergie élevée afin de diminuer l'énergie 
cinétique du système. Autrement dit, l'état fondamental a des projections non nulles sur les $\alpha$ élevés, i.e. cet état permet les fluctuations de charge sur une île au moins d'ordre $e$. Ceci est analogue à l'état antiferromagnétique que l'on trouve en champ magnétique faible dans le modèle de Mott. De même qu'on a alors une corrélation antiferromagnétique des spins des électrons de sites voisins, de même ici on a une anticorrélation d'occupation entre îles voisines: lorsque sur une île un état de $\alpha$ élevé est occupé, cet état est inoccupé sur les îles voisines $\left({ }^{7}\right)$. Cependant, même si on n'a plus blocage de Coulomb individuel, on a encore un "gap" entre le fondamental du système et ses excitations de basse énergie. C'est pourquoi on appelle cette phase : phase de blocage de Coulomb collectif.

- Enfin, lorsque $t>U$, on a une phase où il n'y a plus de gap entre le fondamental et les excitations de basse énergie : il n'y a plus de blocage de Coulomb, on obtient l'équivalent de l'état métallique dans le modèle de Mott à grande concentration d'électrons.

Signalons qu'en présence de fort désordre, il ne sera plus possible d'obtenir la phase de "blocage de Coulomb collectif". En effet, les valeurs de $\epsilon_{\alpha}$ étant distribuées, on est toujours dans le cas " $t$ petit", i.e. toujours dans le cas du blocage de Coulomb individuel. C'est ce cas que nous abordons maintenant.

3.1.3.2. Interactions sur réseau avec désordre. - Middleton et Wingreen [44] considèrent le transport électrique à $T=0$ dans le système décrit par la figure 12 . Des îles métalliques (représentées par des carrés blancs) sont disposées sur un réseau de dimension $d$ et capacitivement couplées entre elles. La capacité entre premiers voisins est prise égale à $C=$ constante et la capacité entre îles non premières voisines est négligée, car le nombre de lignes de champ électrostatique qui relient directement deux îles non premières voisines est très réduit. De plus chaque île est capacitivement couplée à la masse via une capacité $C_{0}$ prise indépendante de l'île.

Les auteurs se placent dans la situation $C \ll C_{0}$, c'est-à-dire que pour chaque île, le couplage capacitif principal est le couplage à la masse via $C_{0}$. En effet, $C$ et $C_{0}$ sont en parallèle, i.e. la capacité effective pour chaque île est $C_{\text {eff. }} \simeq C+C_{0} \simeq C_{0}$. L'avantage de cette limite est que les grains sont presque indépendants : on peut donc négliger les corrélations de charge entre grains non premiers voisins. Pour prendre en compte le désordre (dî aux fluctuations diverses des propriétés des îles), les auteurs introduisent la notion de charge d'offset $q_{i}$ qui dépendant de l'île considérée et qui, dans les simulations numériques, sont tirées au hasard. On peut montrer que chaque charge d'offset $q_{i}$ a pour effet de décaler le dernier niveau d'énergie du grain $i$ de $\sim \frac{q_{i}^{2}}{C_{0}}$. Le système présente donc un fort désordre sur les niveaux d'énergie entre les îles, ce qui, dans le langage du modèle du paragraphe précédent, signifie qu'on est ici dans le cas $t$ petit. Ajoutons que si le désordre en

$\left({ }^{7}\right)$ Poursuivant cette analogie, la phase à petit $t$ (blocage de Coulomb individuel) est analogue à la phase ferromagnétique que l'on trouve dans le modèle de Hubbard à fort champ magnétique. 


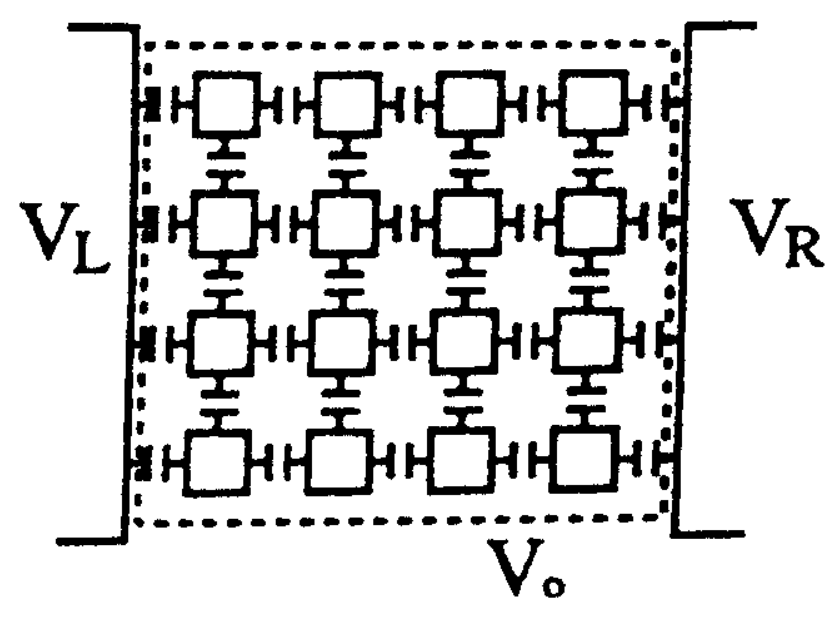

Fig. 12. - Modèle de grains métalliques — représentés par des carrés blancs — couplés par des capacités toutes prises égales à $C$. Chaque île métallique est couplée à la grille - en pointillés, potentiel $V_{0}$ - via une capacité $C_{0}$ constante, non représentée sur la figure. Le désordre est pris en compte par les charges d'offset $q_{i}$, où $0<q_{i}<|e| V_{\mathrm{L}}$ est le potentiel du contact de gauche et $V_{\mathrm{R}}$ celui du contact de droite [44].

[Model of a network of metallic grains - white squares - coupled to the nearest neighboors by capacities all equal to a constant $C$. Each metallic island is coupled to the gate - dashed, potentiel $V_{0}$ - by a constant capacity $C_{0}$ (not showed). Offset charges, $0<q_{i}<|e|$, mimic the effect of disorder. $V_{\mathrm{L}}$ and $V_{\mathrm{R}}$ are respectively the potential of the left and right contacts [44].]

énergie excède $\frac{e^{2}}{C_{0}}$ alors celui-ci sera en partie comblé par l'arrivée d'un nombre entier de charges. C'est pourquoi $0<q_{i}<|e|$.

Partant de là, l'énergie du système vaut :

$$
E=\frac{1}{2} \sum_{\hat{1} \operatorname{les} i, j}\left(Q_{i}+q_{i}\right) C_{i j}^{-1}\left(Q_{j}+q_{j}\right)+V_{\mathrm{L}} Q_{\mathrm{L}}+V_{\mathrm{R}} Q_{\mathrm{R}}+\sum_{\hat{1} \operatorname{les} i} V_{i}^{\text {ext }} Q_{i}
$$

où $Q_{i}$ est le nombre entier de charges se trouvant sur l'île " $i$ " du fait de l'imposition des potentiels $V_{\mathrm{L}}$ et $V_{\mathrm{R}}$ sur les contacts de gauche et de droite qui portent les charges $Q_{\mathrm{L}}$ et $Q_{\mathrm{R}} \cdot C_{i j}^{-1}$ est un élément de la matrice inverse de la matrice capacitance dont on a déjà expliqué qu'elle ne contient que $C$ et $C_{0}$ et que c'est une matrice bande car les capacitances entre îles non premières voisines sont négligeables. Le terme $V_{i}^{\text {ext }}$ est un terme de bord traduisant l'effet sur l'île “ $i$ " des potentiels imposés $V_{\mathrm{L}}, V_{\mathrm{R}}\left(^{8}\right)$. Pour l'expression explicite de ce terme, nous renvoyons à [44].

$\left({ }^{8}\right)$ En effet l'inverse d'une matrice bande n'est pas une matrice bande ce qui implique que chaque île " $i$ " a un terme de couplage à $V_{\mathrm{L}}, V_{\mathrm{R}}$. 
La situation de départ de la simulation numérique est $V_{\mathrm{L}}=V_{\mathrm{R}}$ et bien sûr $Q_{i}=0, \forall i$. Fixant $V_{\mathrm{R}}$ et augmentant $V_{\mathrm{L}}$, les charges vont migrer de gauche à droite dans le réseau de grains. La condition pour qu'une charge puisse tunneler du site " $i$ " au site voisin " $j$ " est que le terme de Coulomb soit plus petit que la différence de potentiel entre ces deux sites, i.e. :

$$
V_{i}>V_{j}+\frac{e}{C_{0}}
$$

Notons que cette condition sera d'autant plus facile à remplir que $q_{i}$ excède $q_{j}$, ainsi que le montre le schéma de la figure 13 . ¿̀ $V_{\mathrm{L}}$ fixé, l'avance des charges est stoppée lorsque en tout point $V_{i}<V_{j}+\frac{e}{C_{0}}$. Puisque l'avance des charges est bloquée le courant ne passe pas : on dit que l'on est au-dessous du voltage critique $V_{\mathrm{C}}$. Donc, en dépit du caractère aléatoire de l'effet tunnel, la configuration statique de charges à n'importe quel voltage inférieur au voltage critique $V_{\mathrm{C}}$ est entièrement définie par la configuration de désordre des charges d'offset $q_{i}$. C'est pourquoi, à une valeur de $V_{\mathrm{L}}$ donnée correspond de manière bijective une seule configuration de charges $Q_{i}$. Nous appellerons "interface" la ligne séparant la zone chargée (où les $Q_{i} \neq 0$ ), de la zone non chargée (où $Q_{i}=0$ ). Lorsque $V_{\mathrm{L}}$ augmente de $\frac{e}{C_{0}}$, une charge est ajoutée en chaque point et l'interface doit donc avancer au moins d'un cran, possiblement de plus d'un cran si le désordre du grain suivant est favorable (si sa charge d'offset est petite). La progression de cette interface lorsque $V_{\mathrm{L}}$ augmente est décrite par la figure 13.

Puisque l'on est à $T=0$, le courant ne pourra commencer à passer que lorsque le point le plus avancé de l'interface sera arrivé au contact de droite. À ce moment là on est dans la situation où :

- $V_{\mathrm{L}}-V_{\mathrm{R}}=V_{\mathrm{C}}$, ce voltage critique traduisant une transition de phase entre une phase où les charges sont liées par l'interaction de Coulomb $\left(V_{\mathrm{L}}-V_{\mathrm{R}}<V_{\mathrm{C}}\right)$ et une phase où il existe des charges libres donc un courant fini à température nulle (état métallique) ;

- un seul chemin $\Gamma_{0}$ conduit le courant, ce chemin étant le meilleur, c'est-à-dire celui qui minimise la somme de tous ses termes de Coulomb. Le chemin $\Gamma_{0}$ (correspondant à une certaine configuration de désordre) est représenté (en noir) sur la figure 14. Il représente des caractéristiques statistiques "duales" de celles de l'interface dont le comportement statistique a été étudié dans le cadre de ce que l'on appelle l'équation Kardar-Parisi-Zhang [15]. Sans entrer dans les détails, signalons que si $\Gamma_{0}$ est aussi peu tortueux c'est parce que les fluctuations des termes de Coulomb sont faibles $\left(0<q_{i}<|e|\right)$. C'est pourquoi l'optimisation de $V_{\mathrm{C}}$ se fait par un chemin peu tortueux car c'est surtout le nombre de capacités rencontrées qui fixe l'énergie totale à fournir. Ceci s'oppose au cas de la résistance globale en régime de Mott étudié au paragraphe 1 . 
(a)

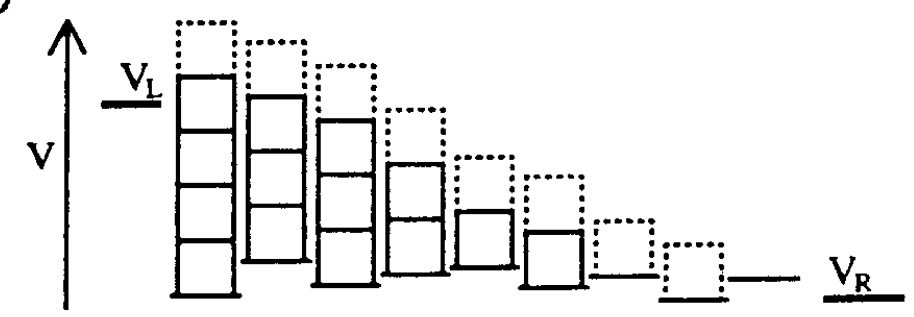

(b)

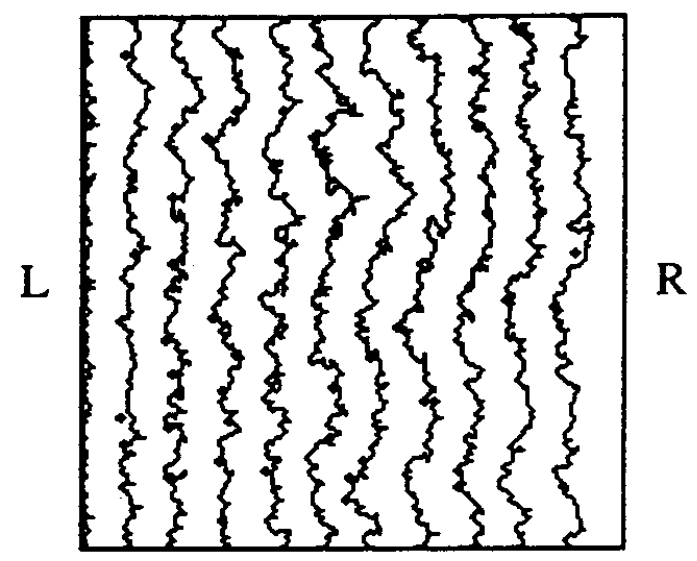

Fig. 13. - a) Schéma des voltages d'îles pour un réseau unidimensionnel au-dessous du voltage seuil de conduction. Chaque carré indique une augmentation de $\frac{e}{C_{0}}$ dans le voltage sur site du fait de l'arrivée d'une nouvelle charge. L'offset relatif entre les voltages est dû aux charges d'offset $0<q_{i}<|e|$. Si le voltage $V_{\mathrm{L}}$ est à nouveau accru de $\frac{\epsilon}{C_{0}}$, les charges progresseront par effet tunnel dans le réseau jusqu'au moment où elles rencontreront une charge d'offset trop grande, comme ceci est figuré par les carrés en pointillé [44]. b) Progression de l'interface "zone chargée-zone neutre" à mesure que la différence de potentiel $V=V_{\mathrm{R}}-V_{\mathrm{L}}$ augmente. La forme de l'interface fluctue à cause du désordre pris en compte par les charges d'offset. Lorsque $V$ atteint $V_{\mathrm{C}}$, le point le plus avancé de l'interface (A) atteint le contact de droite [44].

[a) Schematic view of island voltages in a 1d network below the conduction threshold voltage. Each square indicates an increase of the on-site voltage of $\frac{e}{C_{0}}$ due to the addition of a new charge on the considered grain. Offset charges, $0<q_{i}<|\epsilon|$, are responsible for relative offset voltages. If $V_{\mathrm{L}}$ is further increased of $\frac{e}{C_{0}}$, charges tunnel forward in the network as long as they encounter a offset charge too large (dashed squares). From [44]. b) Progression of the "charged-neutral" interface when the tension $V=V_{\mathrm{R}}-V_{\mathrm{L}}$ increases. The exact shape of the interface fluctuates due to the disorder taken into account by offset charges. When $V$ reaches $V_{C}$, the most advanced point (A) of the interface reaches the right contact. From [44].] 


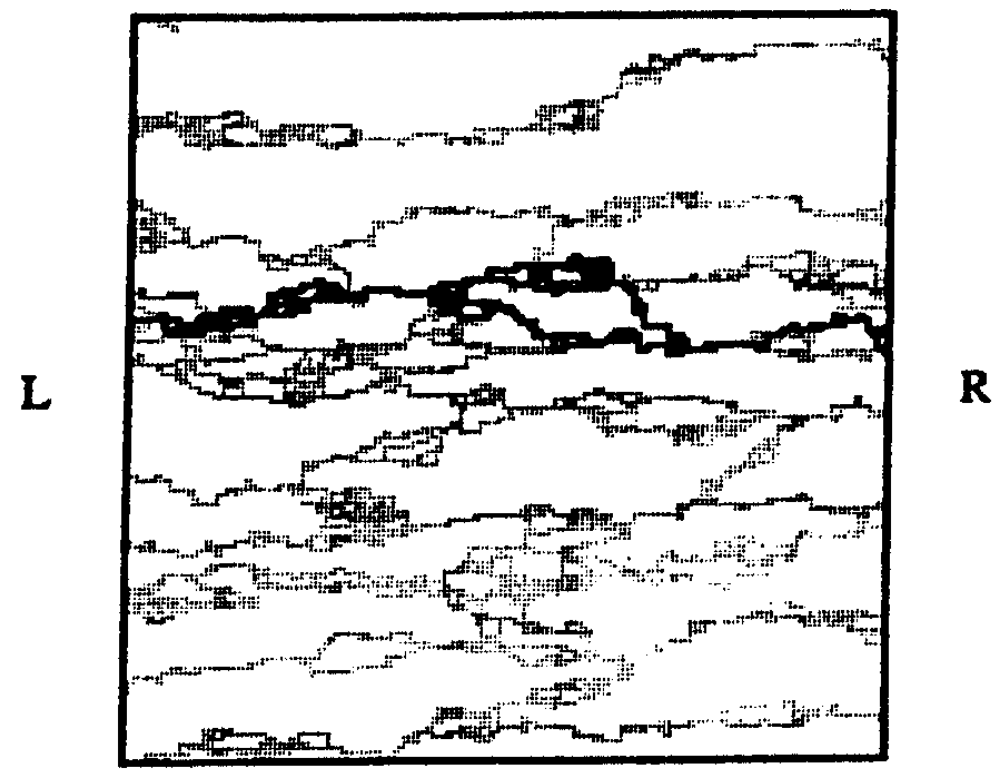

Fig. 14. - En noir, le chemin optimal pour $V$ juste un peu supérieur à $V_{\mathrm{C}}$ : le courant passe alors ultra-majoritairement par ce chemin, la conduction le long de tous les autres étant exponentiellement faible. Ce chemin est bien moins tortueux que celui optimisant la résistance en régime linéaire ( $c f$. Fig. 1) et du même type que celui optimisant la somme de variables distribuées de façon étroite sur un réseau ( $c f$. chemin peu tortueux de la Fig. 1). Pour $\Gamma_{0}$, la longueur divergeant à $V=V_{\mathrm{C}}$ est la distance $\mathcal{L}$ entre points de branchements du chemin. Pour le chemin noir, cette longueur est finie car $V>V_{\mathrm{C}}$. Lorsque $\frac{V-V_{\mathrm{C}}}{V_{\mathrm{C}}}$ devient d'ordre 1 le nombre de chemins optimaux augmente considérablement (cas représenté en gris clair) [44].

[Black: optimal path for $V$ just above the threshold voltage $V_{\mathrm{C}}$ : the electrical current mostly flows along this path, the conduction along all other paths being exponentially small. This path is much less tortuous than the one optimising the global resistance in the linear regime (see Fig. 1) and is of the same kind than the one optimising the sum of random variables narrowly distributed on a network (see Fig. 1). For $\Gamma_{0}$ the diverging length at $V=V_{\mathrm{C}}$ is the distance $\mathcal{L}$ between the branching points along the path. For the black path, $\mathcal{L}$ is finite since $V>V_{\mathrm{C}}$. When $\frac{V-V_{\mathrm{C}}}{V_{\mathrm{C}}} \simeq 1$, the number of optimal paths considerably increases (grey). From [44].]

Bien sûr, à mesure que $V_{\mathrm{L}}-V_{\mathrm{R}}$ dépasse $V_{\mathrm{C}}$, de plus en plus de chemins conduisent. Sur la figure 14, les chemins en gris clair représentent les chemins conducteurs pour un voltage supérieur à $V_{\mathrm{C}}$.

Les propriétés de ces chemins sont les suivantes :

- la longueur critique $\mathcal{L}$ du problème est la distance entre points de branchement sur un chemin. Cette longueur diverge à la transition (i.e. à $V_{\mathrm{C}}$ ) comme 


$$
\mathcal{L} \propto v^{-1} \text { où } v=\left(\frac{V_{\mathrm{L}}-V_{\mathrm{R}}}{V_{\mathrm{C}}}-1\right)
$$

- l'extension transverse $\mathcal{L}_{\perp}$ d'un chemin est $\mathcal{L}_{\perp} \propto \mathcal{L}^{\nu}$ où $\nu_{d=2}=\frac{2}{3}$ alors que $\nu_{d=3}=0,61 \pm 0,015$.

Pour une valeur donnée de $v$, il y a donc un nombre $N_{\perp} \simeq \frac{S_{\perp}}{\mathcal{L}_{\perp}{ }^{d-1}}$ de meilleurs chemins conducteurs, en notant $S_{\perp}$ la surface de l'échantillon orthogonale au courant. Donc :

$$
N_{\perp} \propto \mathcal{L}^{-(d-1) \nu} \propto v^{(d-1) \nu}
$$

Or chacun des $N_{\perp}$ meilleurs chemins est unidimensionnel, ce qui implique que le courant qui y passe est proportionnel à $v$. Il vient donc pour l'échantillon global :

$$
\frac{I}{I_{0}}=v^{\beta} \text { avec } \beta=1+(d-1) \nu_{d}
$$

ce qui amène $\beta_{d=2}=\frac{5}{3}$ et $\beta_{d=3}=2,22 \pm 0,02$. Précisons que $I_{0}$ est le courant qui circule dans l'échantillon lorsque $v=1$, c'est-à-dire lorsque $V_{\mathrm{L}}-V_{\mathrm{R}}=2 V_{\mathrm{C}}$, ce qui constitue la limite supérieure de la région critique. En effet, si a est la taille d'un motif du réseau, on a pour $v=1$ :

$$
\mathcal{L}_{\perp}=\frac{a}{v^{\nu}}=a
$$

ce qui signifie que statistiquement tous les chemins directs conduisent le courant.

Cette théorie a été testée par des travaux récents sur des systèmes modèles bidimensionnels réalisés grâce aux techniques de lithographie les plus modernes [45]. L'accord est bon, les différences entre prédictions et expérience pouvant s'expliquer soit par effets de taille finie soit par effets de température finie. Signalons que Ladieu, Sanquer et Bouchaud ont repris le modèle de Middleton et Wingreen en $\mathrm{y}$ incluant les effets de température finie [13,46]. Ces auteurs montrent la grande pertinence de ce modèle en ce qui concerne les non linéarités d'un alliage amorphe $\mathrm{Y}_{0,2} \mathrm{Si}_{0,8}$ aussi bien sur des films minces $(d=2)$ que épais $(d=3)$. Dans l'interprétation de Ladieu et al. ce sont les fluctuations de $\xi\left(E_{\mathrm{F}}\right)$ qui permettent de définir un réseau analogue à celui de Middleton et al. : les sites de grande longueur de localisation sont assimilés aux îles métalliques, ces sites étant séparés par des régions de petite longueur de localisation assimilées aux barrières isolantes de Middleton et al.. Ceci suggère que la pertinence du modèle de Middleton et al. ne se limite pas aux échantillons modèles réalisés par lithographie, mais que ce modèle peut être utile pour les non linéarités des isolants désordonnés. De plus, en régime linéaire, les auteurs utilisent ce modèle d'îles capacitivement couplées pour rendre compte d'un passage de la loi de Mott $R \sim \exp \left(\frac{T_{0}}{T}\right)^{1 / 4}$ à une loi simplement activée $R \sim \exp \left(\frac{U}{T}\right)$ lorsque la température diminue. 
Comme nous allons le voir dans la suite, les concepts précédents sont très différents de ceux qui ont été utilisés jusqu'ici dans les isolants désordonnés aussi bien lorsqu'il s'est agit de prendre en compte les interactions que les effets non linéaires. En effet, jusqu'au travail de Middleton et Wingreen, aussi bien les interactions que les non linéarités ont été abordées dans un cadre des modèles que nous appellerons "homogènes". Cette hypothèse d'homogénéité est opposée à ce que nous venons d'exposer dans le cadre du blocage de Coulomb comme le révèle l'examen de la figure 13a : la différence de potentiel y est inhomogène car elle est concentrée entre les îles du fait de la différence qualitative entre les îles — parfaitement métalliques — et les barrières qui les entourent — parfaitement isolantes - On pourrait traduire cette différence qualitative en disant que le modèle ci-dessus contient deux longueurs de base: la taille des îles métalliques et celle des régions isolantes. Ceci tranche nettement avec les modèles élaborés bien plus tôt et que nous exposons maintenant.

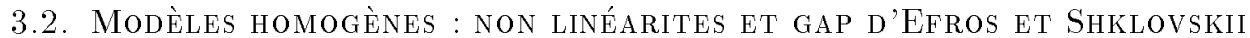

\subsubsection{Modèles homogènes de non-linéarités}

3.2.1.1. Modèle "du champ électrique $\mathcal{E}$ ". - Dans le cas du saut à portée variable, les non-linéarités de la conductance ont été calculées par Larkin et Kmel'nitskii [47]. Ces auteurs calculent de quelle manière la résistance diminue lorsque le voltage d'excitation $V$ augmente. Tant que le champ électrique $\mathcal{E}$ est assez faible pour que la différence de potentiel à l'échelle de $r_{\text {Mott }}$ ne soit pas plus grande que le saut en énergie $E_{\text {Mott }}$ associé au saut de Mott, le saut de Mott reste le même et l'on obtient :

$R_{V}=R_{V=0} \cdot \exp \left(-\frac{e \mathcal{E} r_{\mathrm{Mott}}}{T}\right)$ ceci tant que $: e \mathcal{E} r_{\mathrm{Mot}} \ll E_{\mathrm{Mott}}=\frac{T}{2}\left(\frac{T_{0}}{T}\right)^{\frac{1}{d+1}}$

Bien sûr, lorsque $\mathcal{E}$ augmente trop, le longueur du saut de Mott est affectée et elle commence à diminuer au fur et à mesure que $\mathcal{E}$ augmente. Les auteurs montrent que $R(\mathcal{E})$ est toujours donnée par la même loi que $R(\mathcal{E} \rightarrow 0)$ mais que la température caractéristique de cette loi diminue continûment lorsque $\mathcal{E}$ augmente. Si $\ln \left(R_{V=0}\right) \propto \exp \left(\frac{T_{0}}{T}\right)^{\gamma}$, la seule chose que changera un champ $\mathcal{E}$ donné sera de diminuer $T_{0}$ en le remplaçant par :

$$
T_{0}(\mathcal{E})=T_{0} \cdot\left(1-\frac{e \mathcal{E} \xi}{T}\right)
$$

Ceci est valable aussi bien lorsque $\gamma=\frac{1}{d+1}$ - loi de Mott - que lorsque $\gamma=\frac{1}{2}$ - loi d'Efros et Shklovskii, $c f$. paragr. 3.2.2 - Nous retiendrons donc que à température donnée, la diminution continue de $T_{0}$ avec $\mathcal{E}$ engendre une augmentation exponentielle de la conductance, jusqu'à atteindre un régime indépendant de la température (lorsque $T_{0}(\mathcal{E})=0$ ). Soulignons que, selon notre terminologie, ce modèle est homogène car il ne contient qu'un seule longueur fondamentale : $\xi$. Aucun 
comportement critique n'est attendu en champ électrique contrairement à ce que nous avons exposé dans le modèle de Middleton et Wingreen (paragr. 3.1.3.2).

3.2.1.2. Modèle des électrons chauds. - La loi de Mott — ou d'Efros et Shklovskii, cf. paragr. 3.2.2 - a été très observée à faible champ électrique. Il est donc bien surprenant que la loi de non linéarités énoncée ci-dessus ait beaucoup moins été observée puisqu'elle découle très naturellement de la notion de saut à portée variable. Nous ne connaissons qu'un seul article où la loi de Larkin Kmelnitskii s'est avérée pertinente pour rendre compte des non linéarités expérimentales [48]. Ce fut dans une expérience menée sur Si:P pour $250 \mathrm{mK}<T<500 \mathrm{mK}$. Signalons cependant que la valeur obtenue pour $r_{\text {Mott }}$ à partir des non linéarités fut de $\sim 16 \mu \mathrm{m}$ à $T=300 \mathrm{mK}$, valeur environ 100 fois supérieure aux estimations les plus favorables. Cet écart manifeste n'a jamais reçu d'explication.

Face à ce problème, le modèle le plus utilisé a été celui des "électrons chauds". L'idée centrale est directement héritée des semiconducteurs métalliques : à basse température, les électrons sont mal couplés aux phonons, au point que l'émission de phonons est insuffisante pour dissiper toute l'énergie que le champ électrique communique aux électrons. Leur température s'élève donc au-dessus de celle des phonons. Soulignons tout de suite l'ambiguïté microscopique de ce modèle : dans un métal il est tout à fait permis qu'un électron à $E_{\mathrm{F}}$ change progressivement de niveau d'énergie puisque tous les niveaux sont délocalisés ce qui fait que leur recouvrement est fort. Mais dans le cas d'états localisés, les niveaux proches en énergie sont statistiquement très éloignés, i.e. leur recouvrement est exponentiellement faible. Il est donc bien difficile d'imaginer par quel mécanisme microscopique la température des électrons pourrait s'élever au-dessus de celle des phonons dans un isolant désordonné. En ce sens, le modèle de Larkin Kmelnitskii nous semble prendre en compte ce faible recouvrement entre niveaux localisés puisqu'il garde la notion de passage par effet tunnel entre niveaux localisés.

Soulignons que notre argument n'exclue pas que l'isolant dans son ensemble i.e. phonons et électrons - chauffe par rapport au cryostat en fort champ électrique du fait d'une thermalisation expérimentalement imparfaite qui rend difficile l'évacuation de la puissance injectée. En effet, la conductance thermique $G_{\text {ph.s }}$ entre l'échantillon et le crysotat est toujours finie, et l'on s'attend donc à ce que la différence de température entre le cryostat et un isolant désordonné croisse à mesure qu'augmente la puissance électrique injectée dans ce dernier. On peut illustrer cette idée en commentant le travail de Wang et al. [49] effectué sur du Ge dopé par transmutation neutronique. En jouant sur la forme de l'échantillon et sur celle des électrodes, les auteurs sont capables d'injecter une forte puissance $\mathcal{P}$ dans une région de l'échantillon, et une faible puissance dans une seconde région. Cette dernière se comporte selon la loi de Mott en régime linéaire et sert de thermomètre indiquant la température $T_{\mathrm{ph}}$. de l'échantillon entier car il est raisonnable de négliger la différence de température entre les deux régions. Pour chaque valeur de $\mathcal{P}$, les auteurs extraient la valeur de $T_{\mathrm{ph}}$ à partir de la résistance de la région où la 


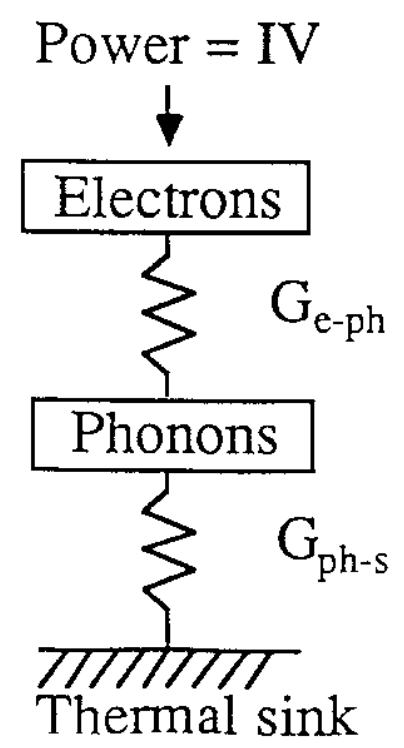

Fig. 15. - Schéma du modèle des électrons chauds. La puissance électrique injectée $\mathcal{P}=I V$ est communiquée aux électrons qui sont couplés aux phonons par une conductance thermique finie $G_{\text {e.ph. }}$. Les phonons sont à leur tour couplés au cryostat ("Thermal Sink") par une impédance thermique finie $G_{\mathrm{ph} . \mathrm{s}}$. D'après [49].

[Schematic representation of the hot electrons model. Electrons receive the power $\mathcal{P}=$ $I V$ and are coupled to phonons by a finite thermal conductance $G_{\text {e.ph. }}$. Phonons are themselves linked to the cryostat ("Thermal Sink") by a finite thermal conductance $G_{\mathrm{ph} . \mathrm{s} .}$. From [49].]

faible puissance est injectée. La corrélation entre $\mathcal{P}$ et $T_{\mathrm{ph}}$. suit la loi :

$$
\mathcal{P}=g_{\mathrm{ph.s}}\left(T_{\mathrm{ph}}^{\beta+1}-T_{\mathrm{s}}^{\beta+1}\right)
$$

où $T_{\mathrm{s}}$ est la température du cryostat ("s" signifiant "heat Sink"), $g_{\mathrm{ph} . \mathrm{s}}$ est une constante reliée à $G_{p h .-s}$ et $\beta$ est une constante. Les auteurs obtiennent $\beta=3,2$ ce qui est très proche de ce que donne le calcul standard de l'impédance thermique entre deux solides en contact $\beta=3$.

S'appuyant sur cette connaissance de $T_{\mathrm{ph}}(\mathcal{P})$, les auteurs cherchent à rendre compte des non linéarités courant tension de la région où la forte puissance $\mathcal{P}$ est injectée. Après avoir constaté l'échec de la loi de Larkin Kmel'nitskii (cf. Sect. précédente), le modèle schématisé sur la figure 15 est adopté. On suppose l'existence d'une impédance thermique $G_{\text {e.ph. }}$ entre les électrons et les phonons. L'écoulement de la puissance électrique des électrons vers le cryostat va donc produire un gradient thermique : $T_{e}>T_{\mathrm{ph}} .>T_{\mathrm{s}}$. où $T_{e}$. est la température des électrons. De même que 


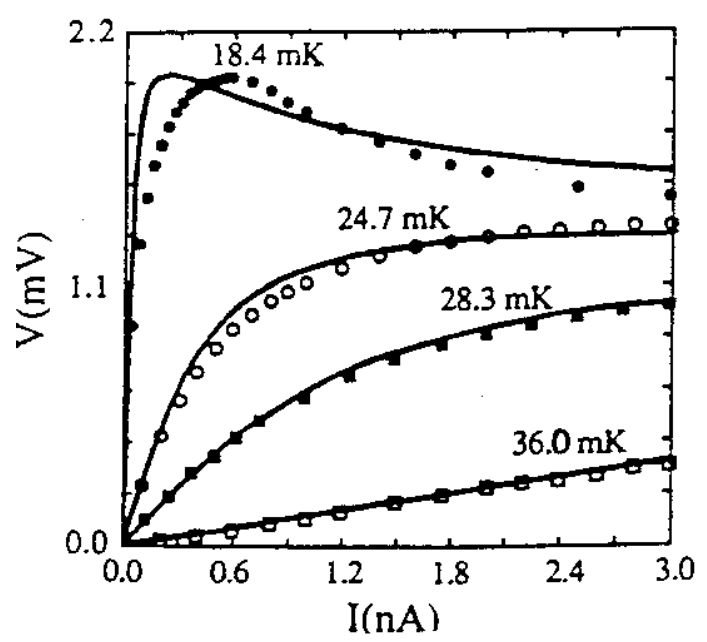

Fig. 16. - Les points sont les non linéarités observées à diverses températures $T_{\Xi}$ du cryostat, indiquées sur le graphe. Les lignes continues sont le meilleur ajustement possible utilisant le modèle des électrons chauds. D'après [49].

[The points are the non linearities experimentally obtained at various temperatures $T_{\Xi}$ of the cryostat. - see $T_{s}$ values on the graph - The continuous lines are the best fit using the hot electrons model. From [49].]

ci-dessus, les auteurs écrivent :

$$
\mathcal{P}=g_{\text {e.ph. }}\left(T_{e}^{\alpha+1}-T_{\mathrm{ph} .}^{\alpha+1}\right)
$$

où $g_{\text {e.ph. }}$ et $\alpha$ sont des constantes dont l'ajustement doit permettre de rendre compte des non linéarités courant tension. En effet, la résistance $R\left(T_{e}.\right)$ s'obtient en remplaçant $T$ par $T_{e}$. dans la résistance en régime linéaire. Il vient donc, en notant par $R_{0}$ une constante de proportionnalité :

$$
V=I R\left(T_{e}\right)=I R_{0} \exp \left(\frac{\mathcal{U}}{T_{e}}\right)^{1 / 2}
$$

où $\mathcal{U}$ et l'exposant $\frac{1}{2}$ sont les marques de la loi d'Efros et Shklovskii ( $c f .3 .2 .2$ ), valable en régime linéaire pour les systèmes Ge considérés ici.

Les deux relations précédentes permettent les ajustements illustrés par la figure 16. La valeur choisie est $\alpha=5$. Cette valeur est aussi celle qui apparaît comme la meilleure pour les non linéarités de films amorphes isolants $\mathrm{Nb}_{x} \mathrm{Si}_{1-x}$ où $x \simeq 0,085$ [50]. À notre connaissance, les valeurs optimales pour $g_{\text {e.ph. }}$ et $\alpha$ varient entre les divers systèmes étudiés. Ces variations ont une très grande importance pour toutes les équipes qui cherchent à utiliser les isolants désordonnés comme détecteurs de particule : il s'agit alors de mesurer l'échauffement produit lors de 
l'absorption d'une particule par un cristal diélectrique sur lequel est fixé un isolant désordonné servant de thermomètre. Toute la question est de savoir alors quel courant maximal peut circuler dans un tel thermomètre sans que la sensibilité en température soit trop dégradée. Dans ce cadre, les non linéarités ont donné lieu à nombre d'études [51] expérimentales ainsi qu'à certains raffinements théoriques comme celui proposé par Perrin [52] qui introduit l'idée que la température des phonons $T_{\mathrm{ph}}$. dépend de leur longueur d'onde.

Il est intéressant de commenter un peu plus l'allure des non linéarités de la figure 16. Le trait le plus marquant est qu'à fort courant $I$ le voltage $V$ augmente bien plus lentement qu'à faible $I$. Ceci est dû à la décroissance de $R\left(T_{e}\right.$. ) lorsque $\mathcal{P}$ - donc $I$ - augmente. Aux plus basses températures $\left(T_{\mathrm{s}}=18,4 \mathrm{mK}\right)$, cette décroissance de $R\left(T_{e}\right)$, lorsque les électrons chauffent, est si forte qu'elle peut dominer le sens de variation de $V$. L'existence d'un maximum dans $V(I)$ est justement le moment où en augmentant $\mathcal{P}$ on décroît plus $R\left(T_{e}\right.$. ) que l'on n'augmente $I$. Soulignons que ce phénomène de résistance différentielle négative doit s'inverser lorsque $T_{e}$ approche $\mathcal{U}$. En effet, $R\left(T_{e}\right.$. ) ne varie alors quasiment plus et la croissance de $I$ domine le sens de variation de $V$. Aux forts courants, on attend donc $V \sim I$. De plus ce comportement doit être "métallique" au sens où il doit très peu dépendre de la température $T_{\mathrm{s}}$ puisque à fort courant $R\left(T_{e}\right.$.) est peu sensible à $T_{e}$. lequel est lui aussi peu dépendant de $T_{\mathrm{s}}$. Ce régime "métallique" à très fort courant est inexploré sur la figure 16, comme quasiment dans toutes les autres études de non linéarités. Or c'est ce régime qui permet d'arbitrer entre le modèle de grains de Middleton Wingreen et le modèle d'electrons chauds. En effet, Middleton et Wingreen, du fait que leur approche est à $T=0$, ne prédisent les non linéarités que pour $V>V_{\mathrm{c}}$, i.e. pour la phase "métallique". Dans ce régime, les prédictions des deux modèles sont pourtant clairement distinctes : $I \propto V$ suivant l'approche "électrons chauds" alors que $I \propto\left(V-V_{c}\right)^{\beta}$ suivant l'approche "Middleton Wingreen".

Pour résumer cette question des non linéarités, rappelons que le modèle du champ électrique de Larkin Kmel'nitskii permet très rarement de rendre compte des non linéarités expérimentales surtout aux températures les plus basses. Le modèle d'électrons chauds a été bien plus utilisé par les expérimentateurs [49-51] car il permet de rendre compte avec un succès certain des courbes expérimentales tout au moins tant qu'on n'explore pas la région des champs très forts. Cependant, ce modèle nous semble microscopiquement ambigu, car il semble passer sous silence le caractère localisé des états électroniques. L'élaboration récente d'un modèle granulaire par Middleton et Wingreen [44] vient relancer le débat, en prédisant des non linéarités clairement distinctes de celles obtenues par l'approche des électrons chauds. Pour que l'expérience puisse arbitrer entre ces deux modèles, il faut que le régime à très fort champ électrique, peu dépendant de la température, soit exploré avec précision. À notre connaissance, ceci n'a été fait qu'une seule fois [13] au sujet d'alliages amorphes $\mathrm{Y}_{0,2} \mathrm{Si}_{0,8}$. La conclusion fut alors en faveur du modèle de Middleton et al.. Il serait très souhaitable que ceci soit fait sur des systèmes très différents.

3.2.2. Gap de Coulomb d'Efros et Shklovskii. - Toujours dans le cadre des modèles homogènes, nous quittons le domaine des non linéarités pour aborder l'effet des 
interactions entre électrons sur le comportement de la résistance en régime linéaire. Efros et Shklovskii ont été les premiers à proposer un modèle prenant en compte les interactions dans les isolants très désordonnés. Ce modèle a été très important car il fut le premier à prédire que la divergence de $R$ à très basse température est plus rapide que ne le prédit la loi de Mott. Cette divergence accélérée a été très souvent observée expérimentalement, au point que certains pensent que la question des interactions est résolue dans les isolants désordonnés !

Considérons donc un ensemble de sites localisés centrés spatialement en $r_{i}$ et dont l'énergie est $E_{i}$. Du fait du désordre les $E_{i}$ sont distribués de site en site. Dans l'état fondamental, les électrons occupent tous les niveaux d'énergie inférieurs au niveau de Fermi $E_{\mathrm{F}}$, les sites d'énergie supérieure étant vides. Par définition de ce qu'est l'état fondamental d'un système, il est clair que toute excitation à partir de cet état doit être d'énergie positive. Calculons le coût énergétique $C_{i, j}$ du transfert d'un électron du site $r_{i}, E_{i} \leq E_{\mathrm{F}}$ au site $r_{j}, E_{j} \geq E_{\mathrm{F}}$, avec $r_{i j}>\xi$ pour que les deux sites soient distincts. On a :

$$
C_{i, j}=E_{j}-E_{i}-\frac{e^{2}}{\epsilon_{0} \epsilon_{r} r_{i, j}}
$$

où $r_{i, j}$ est la distance entre $i$ et $j$ et $\epsilon_{r}$ est la constante diélectrique relative du matériau étudié. Dans l'expression de $C_{i, j}$, toute la surprise est dans le troisième terme. En effet, ce terme représente l'interaction attractive entre l'électron arrivé en $r_{j}$ et le trou que son départ a créé en $r_{i}$.

Ce troisième terme est négatif puisqu'il provient d'une énergie de stabilisation de la paire électron trou. Lorsque les sites sont proches, ce terme est grand. Puisque $C_{i, j}$ doit être positif, cela signifie que les niveaux proches en énergie au voisinage de $E_{\mathrm{F}}$ ne peuvent pas être trop proches dans l'espace. Autrement dit, près du niveau de Fermi il va falloir que la densité d'états soit fonction de l'écart au niveau de Fermi : plus cet écart $\delta E$ va être faible et plus la densité d'états $n(\delta E)$ devra être faible. Sur la base à la fois de simulations numériques et d'un argument intuitif [16], Efros et Shklovskii proposent que :

$$
n(\delta E) \propto(\delta E)^{d-1}
$$

La densité d'états s'annule donc à $E_{\mathrm{F}}$ mais continûment, on n'a pas un vrai gap mais un gap “doux". Si "loin” de $E_{\mathrm{F}}$ la valeur nominale de la densité d'états est $n_{0}$, on obtient l'extension en énergie $\mathcal{G}$ de ce gap "doux" en égalant la relation ci-dessus avec $n_{0}$. $\dot{A}$ trois dimensions, cette extension en énergie du gap "doux" est $\mathcal{G} \propto n_{0}^{1 / 2}$. Une valeur typique dans les semiconducteurs faiblement dopés est que cette extension en énergie est de l'ordre de quelques Kelvins.

Puisque la loi de Mott a été établie en supposant une densité d'états constante autour de $E_{\mathrm{F}}$ cela signifie que lorsque $T<\mathcal{G}$ on n'aura plus la loi de Mott, mais une divergence plus rapide du fait du manque de niveaux autour de $E_{\mathrm{F}}$. Il est facile de reprendre le raisonnement de Mott en y incluant la dépendance fonctionnelle de $n(\delta E)$. C'est ce que font Efros et Shklovskii pour établir leur loi pour la dépendance 
en température de la résistance lorsque $T<\mathcal{G}$. Ils obtiennent :

$$
R \propto \exp \left(\frac{\mathcal{U}}{T}\right)^{1 / 2} \text { où } \mathcal{U} \simeq \frac{e^{2}}{\epsilon_{0} \epsilon_{\mathrm{r}} \xi}
$$

Notons que cet exposant $\frac{1}{2}$ ne dépend plus de la dimension $d$. De plus, comme le suggèrent à la fois nos notations ainsi que l'expression de $\mathcal{U}$, on peut interpréter l'énergie intervenant dans la loi d'Efros et Shklovskii comme l'énergie de charge d'un ilôt métallique de taille $\xi$, i.e. comme l'énergie à fournir pour ajouter un électron sur un site de taille $\xi$ - considéré comme métallique - entouré d'un matériau isolant de constante diélectrique relative $\epsilon_{\mathrm{r}}$. En dépit de ce parallèle entre le blocage de Coulomb et le gap “doux" d'Efros et Shklovskii, notons que dans le premier cas nous avons vu que $R \propto \exp \left(\frac{\mathcal{U}}{T}\right)^{\gamma}$ avec $\gamma=1$, alors que suivant Efros et Shklovskii $\gamma=\frac{1}{2}$. Ceci provient de ce que ces auteurs font l'hypothèse - peu explicite - que les effets de Coulomb sur site - mesurés par $\mathcal{U}$ - sont toujours plus faibles que les effets de désordre dans les isolants désordonnés [53], effets mesurés par $T_{0}$ que l'on a - aux paragraphes 1 et 2 - approximé par $\Delta$ l'écart entre niveaux sur site nous verrons au paragraphe 3.3 que certains auteurs récusent cette approximation en présence d'interactions entre électrons - Quelle que soit la manière retenue pour estimer $T_{0}$, nous pouvons reformuler l'hypothèse d'Efros et Shklovskii en supposant que, partant d'un site $i$ proche de $E_{\mathrm{F}}$, un électron trouve toujours un site $j$ dont le niveau occupé avant le saut $E_{j}$, avant est suffisamment inférieur à $E_{\mathrm{F}}$ pour que lorsque l'électron saute de $i$ en $j$ on ait :

$$
E_{j, \text { après }}=E_{j, \text { avant }}+\mathcal{U}=\text { valeur aussi proche que l'on veut de } E_{\mathrm{F}}
$$

Comme on l'a expliqué précédemment, le terme "valeur aussi proche que l'on veut de $E_{\mathrm{F}}$ " nécessite quand même de vérifier que le fondamental soit stable par rapport aux excitations de basse énergie et en particulier par rapport aux excitations électron trou. Mais encore une fois, dans ces excitations, lorsque un électron arrive sur un site, on ne compte pas l'énergie $\mathcal{U}$ à payer sur site, i.e. on suppose que le désordre en énergie est suffisamment fort pour que $\mathcal{U}$ soit absorbé dans le désordre, ce que nous avons traduit dans l'équation ci-dessus en disant que on trouve toujours un site d'arrivée $j$ tel que “ $E_{j \text {, après }}$ soit aussi proche que l'on veut de $E_{\mathrm{F}}$ ". C'est pourquoi dans le cadre de cette hypothèse, il ne reste plus à prendre en compte que la partie la plus faible de l'interaction, i.e. la partie d'interaction électron-trou $\frac{e^{2}}{\epsilon_{0} \epsilon_{\mathrm{r}} r_{i, j}}$ qui est $\frac{r_{i, j}}{\xi}$ fois plus petite que le terme de Coulomb sur site $\mathcal{U}$. Soulignons que le modèle d'Efros et Shklovskii est bien un modèle homogène puisqu'il ne fait intervenir qu'une seule longueur de base : $\xi$. C'est cette longueur que l'on retrouve dans l'énergie de Coulomb qui intervient dans le gap d'Efros et Shklovskii : $\mathcal{U}=\frac{e^{2}}{\epsilon_{0} \epsilon_{r} \xi}$. Mais du fait de la disparition des termes sur sites on obtient $R=\exp \left(\frac{\mathcal{U}}{T}\right)^{1 / 2}$ au lieu d'obtenir $R=\exp \left(\frac{\mathcal{U}}{T}\right)^{1}$ 
Loi d'Efros et Shklovskii et état de charge du fondamental

De façon générale, Efros et Shklovskii ont toujours affirmé que leur loi ne peut être adaptée qu'au cas des systèmes dont l'état fondamental est chargé. Notons que dans les semi conducteurs faiblement dopés seuls les atomes de dopant apportent des électrons, dont une partie est piégée par les défauts, les effets de la compensation, etc. Les semi conducteurs faiblement dopés comportent donc a priori des sites de localisation vides d'électrons, ce qui rend leur état fondamental chargé. Ce critère d'état fondamental chargé est très important puisqu'il permet d'expliquer pourquoi la loi d'Efros et Shklovskii s'applique aussi dans le cas de certains systèmes granulaires, fort étudiés dans les années soixante dix. Avant d'étudier ceci en détail ( $c f .3 .3$ ), essayons d'expliciter ce critère. Si le fondamental est chargé, il suffit, pour propager le courant, de déplacer les charges qui existent déjà. Autrement dit, il n'y a pas besoin de fournir au système l'énergie $\mathcal{U}$ nécessaire pour créer un site chargé dans un système initialement neutre. Bien sûr, pour que le fondamental soit chargé, il est nécessaire que le désordre en énergie de site à site soit grand devant l'énergie de charge $\mathcal{U}(\xi) \simeq \frac{e^{2}}{\epsilon_{0} \epsilon_{r} \xi}$. En effet, c'est seulement dans ce cas que le système peut supporter des sites chargés puisque, alors, le respect de l'électroneutralité n'est pas ce qui assure que l'ensemble des électrons soit dans la configuration minimale d'énergie, i.e. dans l'état fondamental. On comprend alors facilement que dans l'hypothèse où le désordre l'emporte sur l'énergie de charge on obtienne $\gamma=\frac{1}{2}$, c'est-à-dire que le système peut tirer parti du désordre pour optimiser sa résistance.

En revanche, lorsque $\mathcal{U}$ est l'énergie dominante, l'état fondamental est neutre et on attend $\gamma=1$, ainsi que dans la physique du blocage de Coulomb. Il est alors naturel de se demander si il est possible d'avoir $\gamma=1$ sans que l'état fondamental soit neutre partout. On peut penser en effet qu'il suffit pour avoir $\gamma=1$ qu'il n'existe pas de chemin traversant l'échantillon ne contenant que des sites chargés. Dans ce cas, à suffisamment basse température, $R$ sera dominée par la loi la plus divergente, i.e. par les sauts électroniques dans les régions neutres, et l'on observera $\gamma=1$. Nous reviendrons sur cette question au 3.3. Mais soulignons tout de suite que cette idée permet d'expliquer pourquoi, même dans certaines expériences sur des semi-conducteurs quasi-1d faiblement dopés, outre les aspects spécifiques du saut à portée variable quasi-1d ( $c f$. paragr. 2), il n'est pas surprenant que l'on ait $\gamma=1$ et une physique du blocage de Coulomb. En effet, il suffit que dans le canal quasi-1d on ait une région formée de sites neutres pour que la résistance globale soit dominée par les sauts électroniques dans cette région pour lesquels la résistance locale est simplement activée. C'est ainsi qu'une des toutes premières expériences du genre a été interprétée. Nous voulons parler de l'expérience de Scott-Thomas et al. réalisée à basse température dans un canal quasi-1d de Si dopé [54]. Même si ce système entre dans la classe des systèmes à petit nombre d'électrons, on y observe la physique du blocage de Coulomb - oscillations périodiques suivant la polarisation de la tension de grille - et pas la loi d'Efros et Shklovskii. 
3.2.3. Passage de la loi de Mott à celle d'Efros et Shklovskii. - Aharony, Zhang et Sarachik se sont penchés sur la question du passage de la loi de Mott à celle d'Efros et Shklovskii [55]. Les auteurs introduisent la temperature $T_{x}$ au-dessus de laquelle la loi de Mott est valide, et au-dessous de laquelle la loi d'Efros et Shklovskii est pertinente. L'idée centrale de ce travail est que les lois de Mott et d'Efros-Shklovskii ne sont que les formes assymptotiques d'une loi plus générale. Plus précisément les auteurs proposent que la résistivité $\rho$ d'isolants désordonnés soit donnée par :

$$
\ln \left(\frac{\rho}{\rho_{0}}\right)=A f\left(\frac{T}{T_{x}}\right)
$$

où $A$ et $T_{x}$ dépendant de l'échantillon mais où la fonction $f$ est universelle. Afin de retrouver la loi de Mott et d'Efros-Shklovskii respectivement au-dessus et en dessous de $T_{x}$, les auteurs imposent que $f(x \gg 1) \propto x^{1 / 4}$ et $f(x \ll 1) \propto x^{1 / 2}$. En identifiant le rayon de Mott et celui d'Efros et Shklovskii pour $T=T_{x}$, les auteurs montrent que :

$$
T_{x} \propto \frac{\mathcal{U}^{2}}{T_{0}}
$$

où $T_{0}$ et $\mathcal{U}$ sont les températures caractéristiques respectivement de la loi de Mott et d'Efros-Shklovskii. Il s'agit ensuite de trouver le comportement complet de $f(x)$. Les auteurs repartent de la forme la plus simple de la résistance liant deux sites de localisation :

$$
R_{i j}=\exp \left(\frac{r_{i j}}{\xi}+\frac{E_{i j}}{T}\right)
$$

et proposent de poser

$$
E_{i j}=\frac{\alpha_{1}}{n\left(E_{\mathrm{F}}\right) r_{i j}^{3}}+\frac{\alpha_{2} e^{2}}{\epsilon r_{i j}}
$$

où $\alpha_{1}$ et $\alpha_{2}$ sont des constantes, qui à la fin du raisonnement apparaissent uniquement exprimables en fonction de $A$ et de $T_{x}$. Dans l'équation précédente le premier terme traduit l'écart en énergie dû au désordre. C'est celui utilisé pour dériver le plus simplement la loi de Mott ( $c f$. introduction de cette revue). Le deuxième terme est d'origine coulombienne et ressemble au terme d'interaction électron trou dont nous venons de parler au paragraphe 3.2 .2 , à ceci près qu'il est changé de signe. Ce terme n'a donc aucune justification, mais les auteurs l'utilisent quand même car, utilisé seul il permet formellement - i.e. artificiellement - de retrouver la loi d'Efros et Shklovskii.

Lorsque $r_{i j}$ augmente - i.e. lorsque $T$ décroît - le terme de désordre décroît plus rapidement que celui de Coulomb, alors qu'à haute température c'est le terme de désordre qui domine. L'écriture choisie précédemment pour $E_{i j}$ permet donc aux auteurs de retrouver les deux comportements asymptotiques à $x \gg 1$ (loi de Mott) et $x \ll 1$ (loi d'Efros et Shklovskii). Pour les valeurs intermédiaires de $x$, les auteurs minimisent l'argument de $R_{i j}$ en conservant l'expression complète de $E_{i j}$. Ils obtiennent :

$$
f(x)=\frac{1+\frac{\sqrt{1+x}-1}{x}}{(\sqrt{1+x}-1)^{\frac{1}{2}}}
$$


Les auteurs appliquent avec succès cette prédiction à quelques données expérimentales.

3.3. Unification Possible de La Loi d'Efros ET Shklovskit $\gamma=\frac{1}{2}$ ET DES CAS À ACTIVATION SIMPLE $\gamma=1$ ?. - Les travaux expérimentaux de transport à très basse température sont nombreux, et ils se font l'écho de la question théorique que nous avons posé au paragraphe 3.2 .2 sur la valeur de $\gamma$ aux plus basses températures. En fait, les deux valeurs $\gamma=1$ et $\gamma=\frac{1}{2}$ sont observées. Certaines études mettent en évidence $\gamma=\frac{1}{2}$ aussi bien sur des semiconducteurs [56] que sur les systèmes de métaux granulaires [57] (composés de petits grains métalliques dispersés au sein d'une matrice isolante). En revanche, dans d'autres cas on observe $\gamma=1$ à basse température [46,58]. La distinction que nous avions esquissé au paragraphe 3.2.2 entre les systèmes à petit nombre d'électrons (semiconducteurs dopés) et systèmes à grand nombre d'électrons (métaux granulaires, alliages métal-isolant amorphes) ne correspond donc pas véritablement à chacune des deux valeurs de $\gamma$, comme on aurait pu a priori le penser en suggérant que le terme d'énergie de charge sur site est dominant dans les systèmes à grand nombre d'électrons et négligeable dans les semiconducteurs dopés. Comme nous l'avons suggéré plus haut, il semble que ce soit l'état de charge de l'état fondamental du système qui décide de la valeur de $\gamma$ à basse température, ce qui revient à savoir si l'énergie de désordre entre sites adjacents $T_{0}$ est supérieure ou non à l'énergie de charge $\mathcal{U}$. C'est pour illustrer cette question que nous nous penchons désormais sur le travail de Cuevas, Ortuno et Ruiz [59].

Ces auteurs abordent la physique des milieux granulaires, pour lesquels l'expérience a montré, dès les années 70, un comportement du type Efros et Shklovskii. Depuis ces mesures, une polémique s'est ouverte au sujet de l'interprétation de ce comportement [60]. Cette polémique peut se résumer ainsi. Si l'on considère un grain métallique de diamètre $d_{\mathrm{g}}$, on est tenté d'écrire que le terme de désordre $T_{0}$ en énergie de grain à grain est donné par l'écart $\Delta$ entre niveaux à $E_{\mathrm{F}}$ qui vaut :

$$
\Delta=\frac{1}{n\left(E_{\mathrm{F}}\right) d^{3}}
$$

Pour $d_{\mathrm{g}}=3 \mathrm{~nm}$ et une densité d'états métallique typique, on obtient $T_{0}=\Delta=$ $3 \mathrm{meV}$, ce qui est très inférieur à l'énergie de charge $\mathcal{U}=\frac{e^{2}}{\epsilon d_{\mathrm{g}}}$ qui vaut sensiblement $30 \mathrm{meV}$ pour un tel grain. Si l'on veut expliquer la valeur $\gamma=\frac{1}{2}$ par le raisonnement d'Efros et Shklovskii, il s'agit donc de trouver "d'autres sources de désordre" que le seul écart entre niveaux sur site, afin de rendre le terme de désordre supérieur à l'énergie de charge. C'est ce que firent Efros et Shklovskii [16] en invoquant les fluctuations de travail de sortie liées à l'orientation cristallographique des faces de sortie différente entre les divers grains métalliques. Nous allons voir que l'argumentation de Cuevas et al. est du même type bien qu'utilisant un argument différent.

Les auteurs considèrent une assemblée de grains modélisée par le hamiltonien de 
liaisons fortes suivant:

$$
\mathcal{H}=\sum_{i} \phi_{i} a_{i}^{+} a_{i}+\mathcal{U} \sum_{i} a_{i}^{+} a_{i} a_{i}^{+} a_{i}+\sum_{i} V_{i j} a_{i}^{+} a_{i} a_{j}^{+} a_{j}
$$

où $a_{i}^{+}$est l'opérateur création sur le grain $i$ et $a_{i}$ l'opérateur annihilation correspondant. $\mathcal{U}$ est l'énergie de charge "sur site" définie plus haut, et $V_{i j}=\frac{e^{2}}{\epsilon r_{i j}}$ est le terme de Coulomb entre charges sur des grains éloignés. $\phi_{i}$ est l'énergie du dernier niveau occupé sur le grain $i$. L'argument principal des auteurs est que l'estimation faite ci-dessus pour $T_{0}$ — par le biais de $\Delta$ - sous estime fortement le désordre diagonal présent sur les $\phi_{i}$. Partant de l'idée que la surface des grains métalliques "réels" est très irrégulière, ils utilisent les résultats sur les statistiques de niveaux dans les systèmes chaotiques pour estimer les fluctuations de $\phi_{i}$. Il est en effet établi que ces statistiques sont correctement prédites par l'approche de matrices aléatoires [61]. Les auteurs commencent par considérer les grains isolément les uns des autres. Raisonnant donc à nombre d'électrons constant - puisque $d$ est fixé - , les auteurs trouvent que la fluctuation d'énergie du dernier niveau occupé de grain à grain est bien plus grande que $\Delta$, i.e. que $T_{0} \gg \Delta$. Pour $d_{\mathrm{g}}=3 \mathrm{~nm}$, cette fluctuation est légèrement supérieure à $\mathcal{U}$. Insistons sur le fait que sur chaque grain l'écart entre niveaux est bien d'ordre $\Delta$ mais que la fluctuation de $\phi_{i}$ s'obtient en cumulant tous les écarts de niveaux depuis le bas du spectre en énergie. Pour des grains de $3 \mathrm{~nm}$ de diamètre, contenant tous 2560 électrons disposés sur les $\frac{2560}{2}=1280$ niveaux de plus basse énergie, on obtient $T_{0}$ en évaluant la fluctuation de l'énergie du 1280 ième niveau entre deux grains, et non pas en cherchant l'écart en énergie entre le 1280 ième niveau et le 1281 ième d'un même grain puisque ce dernier écart donne accès à $\Delta$ et non pas à $T_{0}$. Ce raisonnement à nombre d'électrons imposé se justifie par le fait que $\mathcal{U}$ est — pour l'instant - l'énergie dominante du problème puisque pour des grains de $d_{\mathrm{g}}=3 \mathrm{~nm}$ les auteurs trouvent $T_{0} \simeq \mathcal{U} \gg \Delta$. C'est pourquoi, avant que le transfert de charge entre grains n'ait été autorisé, deux grains voisins $i$ et $j$ sont supposés neutres et donc tels que $\phi_{i}-\phi_{j} \simeq T_{0} \gg \Delta$. Qu'arrive-t-il lorsque les grains sont mis en présence les uns des autres ? Par simplicité les auteurs supposent que les grains ne peuvent avoir que trois états de charge : neutre ou bien chargé $\pm e$. Ceci est justifié par le fait que $T_{0}$ et $\mathcal{U}$ sont du même ordre, et que le cas de grains multichargés ne serait envisageable que pour $T_{0} \gg \mathcal{U}$, ce qui demanderait des tailles de grains nettement inférieures au nanomètre.

Un ensemble de valeurs de $\phi_{i}$ est alors tiré au hasard et tous les grains sont supposés neutres. La minimisation de l'énergie du système se fait en deux étapes. En premier lieu, on impose un niveau de Fermi bien défini, i.e. on vérifie que chaque état rempli correspond à une énergie inférieure à $E_{\mathrm{F}}$ et que chaque état vide correspond à une énergie supérieure à $E_{\mathrm{F}}$. L'application de cette contrainte se fait à nombre d'électrons non constant puisqu'elle impose de changer le nombre d'électrons présents sur certains grains, par exemple sur les grains pour lesquels la valeur de $\phi$ tirée au hasard vérifie $\phi+\mathcal{U} \leq E_{\mathrm{F}}$. En second lieu, on cherche à nombre d'électron constant - tous les déplacements de charge entre grains qui peuvent décroître l'énergie totale du système. On obtient ainsi un état "pseudo 
fondamental" puisque sa stabilité vis-à-vis des excitations multiélectroniques n'a pas été vérifiée. Les auteurs signalent ce fait mais en soulignant que, d'après les travaux existant [62], la différence est infime entre la densité d'états obtenue ainsi et celle obtenue en testant la stabilité vis-à-vis des excitations multiélectroniques. Après moyenne sur 1000 configurations différentes d'un réseau de 1000 grains, les auteurs obtiennent la densité d'états autour du niveau de Fermi. Cette densité s'annule bien paraboliquement, avec une courbure légèrement différente de celle prévue par Efros et Shklovskii. La procédure utilisée permet de connaître le pourcentage de grains chargés dans l'état fondamental. Pour les grains de quelques nanomètres de diamètre (taille typique des grains dans les systèmes expérimentaux), la majorité des grains sont ionisés. Ceci permet aux auteurs de suggérer que c'est bien un raisonnement de type Efros et Shklovskii qui permet de rendre compte de la valeur $\gamma=\frac{1}{2}$ dans les métaux granulaires.

Il nous paraît important d'insister sur le fait que les auteurs trouvent que pour les grands grains $\left(d_{\mathrm{g}} \geq 20 \mathrm{~nm}\right)$ le fondamental devient majoritairement neutre, i.e. le pourcentage de grains ionisés $\mathcal{D}$ tend vers 0 . On s'attend donc, à la limite des très grands grains à avoir une résistance simplement activée, i.e. $\gamma=1$. Comment alors penser le passage du cas des petits grains $\gamma=\frac{1}{2}$ à celui des grands grains ? Très intuitivement, on aurait tendance à comparer la distance $l_{\text {e.e. entre grains }}$ chargés $l_{\text {e.e. }} \simeq d_{\mathrm{g}} \mathcal{D}^{-1 / d}$ à la taille des sauts électroniques $r_{\mathrm{E}}$.s. prévus par la loi d'Efros et Shklovskii $r_{\text {E.S. }} \sim\left(\frac{\mathcal{U}}{T}\right)^{1 / 2}$ avec comme on l'a dit au paragraphe 3.2 .2 $\mathcal{U} \simeq \frac{e^{2}}{\epsilon d_{\mathrm{g}}}$. On serait alors tenté de suggérer que dans les cas des grands grains, $\mathcal{D}$ est suffisamment petit pour qu'il existe un domaine de température tel que :

$$
l_{\text {e.e. }} \gg r_{\mathrm{E} . \mathrm{S}}
$$

Tant que cette inégalité est respectée, on peut penser que tout chemin traversant l'échantillon comporte forcément des sauts entre grains neutres ce qui entraîne $\gamma=$ 1, puisque la résistance globale est alors dominée par les sauts les plus divergents. Aux plus basses températures, l'inégalité ci-dessus n'est plus vérifiée du fait de la

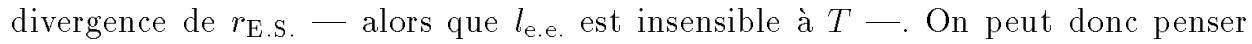
que la loi d'Efros et Shklovskii devient pertinente au-dessous d'une température d'autant plus basse que $\mathcal{D}$ est petit, i.e. que $d_{\mathrm{g}}$ est grand.

$\dot{A}$ notre connaissance, cette idée de passage possible entre $\gamma=1$ et $\gamma=\frac{1}{2}$ n'a pas été exploitée jusqu'ici. Le raisonnement que nous venons de présenter est donc hautement spéculatif et ne peut servir au mieux que de piste indicative. Soulignons que cet argument à l'avantage de présenter la loi d'Efros et Shklovskii et celle où $\gamma=1$ comme des cas limites envisageables au sein du même modèle : celui d'un milieu de grains métalliques dans une matrice isolante. Le cas d'Efros et Shklovskii serait celui des petits grains, le cas des grands grains présentant un comportement simplement activé tant que la température n'est pas inférieure à une température limite qui est extrêmement petite lorsque les grains sont grands. Comme l'ont suggéré Ladieu, Sanquer et Bouchaud [13,46], dans les isolants désordonnés cette 
granularité pourrait être due aux fluctuations de $\xi\left(E_{\mathrm{F}}\right)$. Le cas des grands grains serait donc celui où le système comporte des états à grandes longueurs de localisation, i.e. le cas des isolants très près de la transition. Ces isolants auraient une résistance activée $\gamma=1$ jusqu'à une température limite très basse, impossible à atteindre expérimentalement, ce qui donnerait l'impression que ces systèmes ne suivent jamais la loi d'Efros et Shklovskii. Pour des systèmes plus loin de la transition, la température limite serait bien plus élevée, au point que l'on ne verrait plus que la loi d'Efros et Shklovskii. Ce point de vue aurait l'avantage d'unifier l'interprétation des cas expérimentaux qui présentent $\gamma=1$ et ceux qui suivent la loi d'Efros et Shklovskii.

Au moment de conclure cette dernière partie, soulignons que le problème des interactions électron-électron dans les isolants est un des problèmes les plus débattus de la physique du solide. Le champ des questions ouvertes relatives à l'isolant de Mott est encore très vaste. En témoigne la richesse de la physique du blocage de Coulomb - domaine toujours actuel en dépit des nombreux travaux qu'il a suscité - et qui ne constitue pourtant qu'un cas particulier du problème de Mott. Dans les isolants désordonnés la situation est encore plus difficile puisqu'il s'agit de penser le rapport entre les effets de désordre - difficiles en eux mêmes - et les effets d'interaction très débattus. Signalons à ce propos un article de revue récent sur la transition métal-isolant dans les systèmes à la fois en fort désordre et avec interactions entre électrons [63]. De façon générale, la prise en compte des interactions de Coulomb accélère la divergence de la résistance - par rapport à la loi de Mott puisque l'on obtient $\gamma \geq \frac{1}{2}$. Notons que cette divergence plus rapide apparaît aux plus basses températures, c'est-à-dire lorsque $E_{\text {Mott }}$ est suffisamment petit pour etre affecté fortement par la prise en compte du terme de Coulomb. Nous avons vu que si le terme de Coulomb se réduit à l'interaction électron trou, la loi d'Efros et Shklovskii est pertinente et $\gamma=\frac{1}{2}$. En revanche, si les termes d'énergie de charge sur site entrent en jeu, on attend $\gamma=1$, c'est-à-dire une loi plus divergente que celle d'Efros et Shklovskii car les interactions donnent alors leur pleine mesure. Devant la foule de questions encore ouvertes dans ces systèmes alliant à la fois un fort désordre et de fortes interactions, nous ne pouvons que souhaiter un grand effort théorique qui permette de rendre compte de façon unifiée des diverses situations expérimentales.

\section{Conclusion}

Finalement, la loi de Mott représente la meilleure optimisation possible dans un isolant désordonné, sauf à considérer des systèmes à la fois très courts et très larges dont la conduction est dominée par des chemins très rares et meilleurs conducteurs que l'optimum de Mott. Comme toutes les meilleures optimisations, la loi de Mott est fragile puisqu'elle exige à la fois une géométrie macroscopique et en même temps que les interactions sur site soient négligeables. Nous pouvons donc esquisser un rapprochement entre les effets de la géométrie quasi $1 \mathrm{~d}$, développés dans la deuxième partie, et les effets d'interaction : dans les deux cas l'électron ne peut plus complètement tirer partie du désordre pour optimiser sa résistance et 
l'on obtient alors, à basse température, soit $\gamma=1$ soit $\gamma=\frac{1}{2}$, ce dernier cas correspondant à la prédiction d'Efros et Shklovskii où le désordre joue encore un rôle majeur. En ce qui concerne les systèmes quasi-1d, c'est le confinement du chemin de conduction à une échelle très inférieure à $\xi_{\mathrm{p}}$ qui empêche ce chemin de se convulser suffisamment pour que chaque saut réalise au moins l'optimisation de Mott. En ce qui concerne les systèmes à fortes interactions, le caractère incontournable des termes de Coulomb rend impossible que l'amplitude en énergie des sauts électroniques tende vers 0 à basse température, comme l'exige la loi de Mott. La loi d'Efros et Shklovskii représente le cas intermédiaire où le désordre participe encore fortement à l'optimisation de la résistance même aux plus basses températures.

L'analogie esquissée entre les effets de dimensionnalité et ceux des interactions ne doit cependant pas nous égarer : dans le premier cas l'essentiel de ce qui a été mesuré a reçu une interprétation. Par des mesures plus fines on peut espérer enrichir l'interprétation en testant des points encore peu établis : par exemple la notion de fluctuation quantique tronquée. Cependant il est peu probable que de telles mesures aboutissent à remettre en cause les éléments majeurs des modèles, i.e. les arguments statistiques utilisés pour construire le chemin de conduction.

En revanche au sujet des interactions de nets progrès restent à accomplir aussi bien du point de vue théorique qu'expérimental. Nous avons tenté de distinguer les modèles homogènes mettant l'accent sur les effets de désordre, des modèles granulaires où le rôle des interactions est plus explicitement pris en compte. Nous avons suggéré qu'il est possible que le cadre des modèles granulaires soit assez vaste pour, à la limite des petits grains redonner le cas où le désordre prime, et à la limite des grands grains décrire le cas où ce sont les interactions qui ont le rôle principal. Du point de vue expérimental, nous pensons que le plus urgent consiste à confronter les différents modèles aux mêmes données expérimentales. Ceci a été peu fait, en partie à cause du décalage entre les époques où les diverses approches ont été élaborées. Ceci demande que de nouvelles mesures soient effectuées en mettant l'accent sur les cas où les différentes prédictions sont les plus distinctes. La mesure du comportement non linéaire - y compris en champ électrique très fort — à diverses températures semble être le complément indispensable des nombreuses études faites à faible champ électrique. C'est donc à l'ensemble de la courbe couranttension et à son évolution en température qu'il convient de confronter les différents modèles.

\section{Bibliographie}

[1] Anderson P.W., Phys. Rev. 109 (1958) 1942.

[2] Landauer R., Localization, interaction and transport phenomena, G. Bergmann and Y. Bruynseraede Eds. (Springer Verlag, Heidelberg, 1985) p. 38. 
[3] Efetov K., Zh. Eksp. Teor. Fiz. 78 (1980) 2017 [Sov. Phys. JETP 51 (1980) 1015] ; Adv. Phys. 32 (1983) 53 ; Efetov K., Larkin A.I., Zh. Eksp. Teor. Fiz. 85 (1983) 764 [Sov. Phys. JETP 58 (1983) 444].

[4] N'guyen V.L., Spivak B.Z., Shklovskii B.I., Pis'ma Zh. Eksp. Theor. Fiz. 41 (1985) 35 [J.E.T.P. Lett. 41 (1985) 42] ; Zh. Eksp. Teor. Fiz. 89 (1985) 11 ; Shklovskii B.I., Spivak B.Z., Hopping Transport in Solids (Pollak, Shklovskii Eds., North Holland, 1991).

[5] Kardar M., Medina E., Shapir Y., Wang W.S., Phys. Rev. Lett. 62 (1989) 941 ; 64 (1990) 1816 ; Wang X.R., Shapir Y., Medina E., Kardar M., Phys. Rev., B42 (1990) 4559 ; Medina E., thèse M.I.T. (1991) ; pour une revue complète sur les chemins dirigés voir : Medina E., Kardar M., Phys. Rev. B46 (1992) 9984.

[6] Zhao H.L., Spivak B.Z., Gelfand M.P., Feng S., Phys. Rev. B44 (1991) 10760.

[7] Pichard J.L., Sanquer M., Slevin K., Debray P., Phys. Rev. Lett. 65 (1990) 1812 ; Pichard J.L., Sanquer M., Physica A 167 (1990) ; Pichard J.L., in "Quantum Coherence in Mesoscopic Systems", B. Kramers Ed. (NATO Adv. Studies, Plenum New-York) ; Pichard J.L., Sanquer M., in "Quantum Chaos", G. Casati and B. Chirikov Eds. (Cambridge University Press) ; Pichard J.L., Zanon N., Imry Y., Stone A.D., J. Phys. France 51 (1990) 587 ; Stone A.D., Mello P.A., Muttalib K.A., Pichard J.L., Mesoscopic Phenomena in Solids, B.L. Al'tshuler, P.A. Lee, R.A. Webb Eds. (Elsevier Sciences Publishers B.V., 1991) Chap. 9.

[8] Bouchaud J.P., J. Phys. I France 1 (1991) 985 ; Bouchaud J.P., Sornette D., Europhys. Lett. 17 (1992) 721.

[9] Mott N.F., J. Non-Crystal. Solids 1 (1968) 1.

[10] Ambegaokar V., Halperin B.I., Langer J.S., Phys. Rev. B4 (1971) 2612 ; Pollak M., J. Non-Cryst. Solids 11 (1972) 1 ; Shklovskii B.I., Efros A.L., Zh. Eksp. Teor. Fiz. 60 (1971) 867 [Sov. Phys. JETP 33 (1971) 468].

[11] Feng S., Pichard J.L., Phys. Rev. Lett. 67 (1991) 753 ainsi que ses références.

[12] Pollak M., J. Non-Crystal. Solids 11 (1972) 1 ; Knotek M., Pollak M., J. Non-Crystal. Solids 8-10 (1972) 505.

[13] Ladieu F., Thèse Paris 6, Octobre 1994.

[14] Skal A.S., Shklovskii B.I., Fiz. Tekh. Poluprovodn. 8 (1974) 1586 [Sov. Phys. Semicond. 8 (1975) 1029]; Stanley H.E., J. Phys. A 10 (1977) C221 ; Coniglio A. Phys. Rev. Lett. 46 (1981) 250.

[15] Kardar M., Parisi G., Zhang Y.C., Phys. Rev. Lett. 56 (1986) 889 ; Maritan A., Banavar J.R., Phys. Rev. Lett. 72 (1994) 1451 ; voir aussi [44] et ses références.

[16] Shklovskii B.I., Efros A.L., Electronic Properties of doped semiconductors, Springer Series in Solid State Sciences 45 (Springer-Verlag, 1984). Pour la loi de Mott voir Chapitre 9, pages 202 à 208 ; pour le gap de Coulomb voir Chapitre 10, pages 228 à 240 .

[17] Howard W.E., Fang F.F., Solid State Electron. 8 (1965) 82 ; Milliken F.P., Ovadyahu Z., Phys. Rev. Lett. 65 (1990) 911 ; Popovic D., Fowler A.B., Washburn S., Stiles P.J., Phys. Rev. B42 (1990) 1759 ; M. Sanquer, non publié. 
[18] Shahar D., Ovadyahu Z., Phys. Rev. Lett. 64 (1990) 2293.

[19] Raikh M.E., Ruzin I.M., JETP Lett. 43 (1986) 562 [Pis'ma Zh. Eksp. Teor. Fiz. 43 (1986) 437] ; Raikh M.E., Ruzin I.M., Sov. Phys. JETP 65 (1987)1273 [Zh. Eksp. Teor. Fiz. 92 (1987) 2257] ; voir aussi l'article du livre cité en [27].

[20] Kurkijarvii J., Phys. Rev B8 (1973) 922.

[21] Lee P.A., Phys. Rev. Lett. 53 (1984) 2042.

[22] Serota R.A., Kalia R.K., Lee P.A., Phys. Rev. B33 (1986) 8441.

[23] Fowler A.B., Harstein A., Webb R.A., Phys. Rev. Lett. 48 (1982) 196 ; Wheeler R.G., Choi K.K., Goel A., Wisnieff R., Prober D.E., Phys. Rev. Lett. 49 (1982) 1694 ; Skopol W.J., Jackel L.D., Hu E.L., Howard R.E., Fetter L.A., Phys. Rev. Lett. 49 (1982) 951 ; Kwasnick R.F., Kastner M.A., Melgailis J., Lee P.A., Phys. Rev. Lett. 52 (1984) 224 ; Webb R.A., Harstein A., Wainer J.J., Fowler A.B., Phys. Rev. Lett. 54 (1985) 1577.

[24] Ladieu F., Mailly D., Sanquer M., J. Phys. I France 3 (1993) 2321 ; Poirier W., Sanquer M., Mailly D., in "Hopping and related phenomena", Proceedings of 6th international conference on H.R.P., O. Millo et Z. Ovadyahu Eds., Racah Institute of Physics, the Hebrew university, Jerusalem, p. 309.

[25] Azbel M.Y., Harstein A., DiVincenzo D.P., Phys. Rev. Lett. 52 (1984) 1641. La critique du point de vue de cet article avait d'abord été émise dans [26].

[26] Stone A.D., Lee P.A., Phys. Rev. Lett. 54 (1985) 1196.

[27] Raikh M.E., Ruzin I.M., Sov. Phys. JETP 68 (1989) 642 ; voir aussi page 315 et les suivantes dans "Mesoscopic Phenomena in Solids", B.L. Altshuler, P.A. Lee, R.A. Webb Eds. (Elsevier Science Publisher, 1991).

[28] Ladieu F., Bouchaud J.P., J. Phys. I France 3 (1993) 2311.

[29] Sivan U., Imry Y., Phys. Rev. B35 (1987) 6074.

[30] voir par exemple: Bouchaud J.P., Georges A., Phys. Rep. 195 (1990) 127, et Appendice A de $\boldsymbol{J}$. Phys. II France 1 (1991) 1465.

[31] Avishaï Y., Pichard J.L., Muttalib K.A., J. Phys. I France 3 (1993) 2342.

[32] Orlov A.O. et al., JETP Lett. 43 (1986) 540 [Pis'ma Zh. Eksp. Teor. Fiz. 43 (1986) 421] ; Laiko E.I., Orlov A.O., Savchenko A.K., Il’ichev E.A., Poltoratskii E.A., Sov. Phys. JETP 66 (1987) 6 [Zh. Eksp. Teor. Fiz. 93 (1987) 2204]; Orlov A.O., Savchenko A.K., Koslov A.V., Solid State Commun. 72 (1989) 743.

[33] Popovic D., Fowler A.B., Washburn S., Stiles P.J., Phys. Rev. B42 (1990) 1759.

[34] Popovic D., Fowler A.B., Washburn S., Phys. Rev. Lett. 67 (1991) 2870.

[35] Glazman L.I., Matveev K.A., Sov. Phys. JETP 67 (1988) 1276.

[36] Mott N.F., Proc. Phys. Soc. A 62 (1949) 416 ; voir aussi Can. J. Phys. 34 (1956) 1356.

[37] Edwards P.P., Sienko M.J., Phys. Rev. B17 (1978) 2575 ; voir aussi Mott N.F., Kaveh M., Adv. Phys. 34 (1985) 329.

[38] Georges A., Kotliar G., Phys. Rev. B45 (1992) 6479 ; Georges A., Kotliar G., Si Q., Int. J. Mod. Phys. B 6 (1992) 705.

[39] Laloux L., Georges A., Krauth W., Phys. Rev. B50 (1994) 3092.

[40] Kawabata A., Physica A 204 (1994) 359 : le résultat central de ce travail est 
le calcul de la valeur de la résistance minimale $R_{\min }$ que les barrières tunnels doivent avoir pour qu'un film granulaire présente du blocage de Coulomb. L'auteur trouve $R_{\min } \sim h / e^{2}$, cette valeur étant indépendante de l'énergie de charge $E_{\mathrm{C}}$. En revanche, le calcul mené pour estimer $E_{c}$ nous semble douteux car le résultat est $E_{c}=N(N-1) \frac{e^{2}}{C}$ où $N$ est le nombre d'électrons en excès sur l'île donne $E_{c}(N=1)=0$. Ceci nous semble contredire les résultats des expériences où le blocage de Coulomb est obtenu sur une seule île. Il nous semble que le raisonement utilisé oublie de prendre en compte la polarisation de l'île lorsque l'on approche de l'infini une seule charge en excès. On peut lire à ce sujet la thèse de C. Pasquier ou de H. Pothier [41].

[41] D'excellentes thèses sont disponibles sur le sujet du blocage de Coulomb. Exemples (non exhaustifs) : H. Pothier, thèse Paris 6 (1991) ; C. Pasquier, thèse Paris 11 (1993) ; Ph. Lafarge, thèse Paris 6 (1993).

[42] Pour des revues sur le blocage de Coulomb dans les métaux : Likharev K.K., IBM J. Res. Dev. 32 (1988) 144 ; ou encore : Averin D.V., Likharev K.K., in "Mesoscopic Phenomena in Solids, B.L. Alt'shuler, P.A. Lee, R.A. Webb Eds., (Elsevier, Amsterdam, 1991). Pour une revue sur le blocage de Coulomb dans les semi-conducteurs : van Houten H., Beenaker C.W., Staring A.A.M., in "Single Charge Tunneling", NATO Advanced Study Institute, Series B : Physics, H. Grabert et M.H. Devoret Eds. (Plenum, New York, 1991) ; pour un article récent contenant de nombreuses références : Beenaker C.W., Phys. Rev. B44 (1991) 1646.

[43] Stafford C.A., Das Sarma S., Phys. Rev. Lett. 72 (1994) 3590.

[44] Middleton A.A., Wingreen N.S., Phys. Rev. Lett. 71 (1993) 3198.

[45] Duruoz C.I., Clarke R.M., Marcus C.M., Harris J.S., Phys. Rev. Lett. 74 (1995) 3237 ; Rimberg A.J., Ho T.R., Clarke J., ibid. 74 (1995) 4714 ; Lafarge P., Meindersma J.J., Mooij J.E., to be published in : Macroscopic Quantum Phenomena and Coherence in Superconducting Networks, C. Giovannella Ed. (World Scientific, Singapore, 1995).

[46] Ladieu F., Sanquer M., Bouchaud J.P., Phys. Rev. B53 nº3 (1996).

[47] Larkin A.I., Khmel'nitskii D.E., Sov. Phys. JETP 56 (1982) 647 [ Zh. Eksp. Teor. Fiz. 83 (1982) 1140].

[48] Rosenbaum T.F., Andres K., Thomas G.A., Solid State Commun. 35 (1980) 663.

[49] Wang N., Wellstood F.C., Sadoulet B., Haller E.E., Beeman J., Phys. Rev. B41 (1990) 3761.

[50] voir par exemple : Dumoulin L., Bergé L., Lesueur J., Bernas H., Chapellier M., Compte-rendu de "Low Temperature Detectors" n ${ }^{\circ} 5, J$. Low Temperature Phys. 93 (1993) 301 ; mêmes auteurs, Compte-rendu de "Low Temperature Detectors" $\mathrm{n}^{\circ} 6$, à paraître.

[51] Pour un tour d'horizon des expériences en cours : Compte-rendu de "Low Temperature Detectors" no 5, J. Low Temperature Phys. 93 (1993) 287-433.

[52] Perrin N., Compte-rendu de "Low Temperature Detectors" $n^{\circ}$ 5, J. Low Temperature Phys. 93 (1993) 313. 
[53] Spivak B.Z., communication privée.

[54] Scott-Thomas J.H.F. et al., Phys. Rev. Lett. 62 (1989) 583.

[55] Aharony A., Zhang Y., Sarachik M.P., Phys. Rev. Lett. 68 (1992) 3900.

[56] Exemples récents : Zhang Y., Dai P., Levy M., Sarachik M.P., Phys. Rev. Lett. 64 (1990) 2687 ; Agrinskaya N.V., Aleshin A.N., Fiz. Tverd. Tela (Leningrad) 31 (1989) 277 [Sov. Phys. Solid State 31 (1989) 1996].

[57] Davies J.H., Franz J.R., Phys. Rev. Lett. 57 (1986) 475 ; Hollinger G., et al., Phys. Rev. B32 (1985) 1987 ; Hill M.D., Egdell R.G., J. Phys. C 16 (1983) 6205 ; Weng S.L., Moehlecke S., Strongnin M., Zangwill A., Phys. Rev. Lett. 50 (1983) 1795 ; Abeles B., Sheng P., Coutts M.D., Arie Y., Adv. Phys. 24 (1975) 407.

[58] Chandrasekhar V., Ovadyahu Z., Webb R.A., Phys. Rev. Lett. 67 (1991) 2862 ; Hernandez P., Ladieu F., Sanquer M., Mailly D., Physica B 194-196 (1994) 1141-1142 ; Kim J.J., Lee H.J., Phys. Rev. Lett. 70 (1993) 2798.

[59] Cuevas E., Ortuno M., Ruiz J., Phys. Rev. Lett. 71 (1993) 1871.

[60] Chui S.T., Phys. Rev. B 43 (1991) 14274 ; Sheng P., Philos. Mag. B 65 (1992) 357 ; Pollak M., Adkins C.J., Philos. Mag. B 65 (1992) 855.

[61] Brody T.A., Flores J., French J.B., Mello P.A., Pandey A., Wong S.S., Rev. Mod. Phys. 53 (1981) 385 ; Efetov K.B., Adv. Phys. 32 (1983) 53 ; Bohigas O., Giannoni M.J., Schmit C., Phys. Rev. Lett. 52 (1984) 1 ; Al'tshuler B.L., Shklovskii B.I., Sov. Phys. JETP 64 (1986) 1 ; Zimmermann Th. et al., Phys. Rev. Lett. 61 (1988) 3 ; Akkermans E., Montambaux G., Phys. Rev. Lett. 68 (1992) 642 .

[62] Baranovskii S.D., Efros A.L., Gelmont B.L., Shklovskii B.I., J. Phys. C 12 (1979) 1023 ; Davies J.H., J. Phys. C 17 (1984) 3031.

[63] Belitz D., Kirkpatrick T.R., Rev. Mod. Physics 66 (1994) 261. 